\title{
Meta-Analysis of Carbohydrate Solution Intake during Prolonged Exercise in Adults: From the Last $45+$ Years' Perspective
}

\author{
Dimitrios I. Bourdas ${ }^{1, * \mathbb{C}}$, Athanasios Souglis ${ }^{2}$, Emmanouil D. Zacharakis ${ }^{2}{ }^{\mathbb{D}}$, Nickos D. Geladas ${ }^{1}$ \\ and Antonios K. Travlos ${ }^{3}$ (D) \\ 1 Section of Sport Medicine \& Biology of Exercise, School of Physical Education and Sports Science, \\ National and Kapodistrian University of Athens, 41 Ethnikis Antistasis, 17237 Athens, Greece; \\ ngeladas@phed.uoa.gr \\ 2 Section of Didactics and Coaching in Sport Games, School of Physical Education \& Sport Science, National \\ and Kapodistrian University of Athens, 41 Ethnikis Antistasis, 17237 Athens, Greece; \\ asouglis@phed.uoa.gr (A.S.); emzach@phed.uoa.gr (E.D.Z.) \\ 3 Department of Sports Organization and Management, Faculty of Human Movement and Quality of Life \\ Sciences, University of Peloponnese, Efstathiou and Stamatikis Valioti \& Plataion Avenue, \\ 23100 Tripoli, Greece; atravlos@uop.gr \\ * Correspondence: dbourdas@phed.uoa.gr; Tel.: +30-6977358900
}

\section{check for} updates

Citation: Bourdas, D.I.; Souglis, A. Zacharakis, E.D.; Geladas, N.D.; Travlos, A.K. Meta-Analysis of Carbohydrate Solution Intake during Prolonged Exercise in Adults: From the Last 45+ Years' Perspective. Nutrients 2021, 13, 4223. https:// doi.org/10.3390 nu13124223

Academic Editors:

Maria Luz Fernandez and Sébastien Bailly

Received: 12 October 2021

Accepted: 22 November 2021

Published: 24 November 2021

Publisher's Note: MDPI stays neutral with regard to jurisdictional claims in published maps and institutional affiliations.

Copyright: (c) 2021 by the authors. Licensee MDPI, Basel, Switzerland. This article is an open access article distributed under the terms and conditions of the Creative Commons Attribution (CC BY) license (https:/ / creativecommons.org/licenses/by/ $4.0 /)$.

\begin{abstract}
Carbohydrate ( $\mathrm{CHO}$ ) supplementation during prolonged exercise postpones fatigue. However, the optimum administration timing, dosage, type of $\mathrm{CHO}$ intake, and possible interaction of the ergogenic effect with athletes' cardiorespiratory fitness (CRF) are not clear. Ninety-six studies (from relevant databases based on predefined eligibility criteria) were selected for meta-analysis to investigate the acute effect of $\leq 20 \% \mathrm{CHO}$ solutions on prolonged exercise performance. The between-subject standardized mean difference [SMD = ([mean post-value treatment group-mean post-value control group]/pooled variance)] was assessed. Overall, SMD [95\% CI] of $0.43[0.35,0.51]$ was significant $(p<0.001)$. Subgroup analysis showed that SMD was reduced as the subjects' CRF level increased, with a $6-8 \%$ CHO solution composed of GL:FRU improving performance (exercise: $1-4 \mathrm{~h}$ ); administration during the event led to a superior performance compared to administration before the exercise, with a 6-8\% single-source $\mathrm{CHO}$ solution increasing performance in intermittent and 'stop and start' sports and an $\sim 6 \%$ CHO solution appearing beneficial for 45-60 min exercises, but there were no significant differences between subjects' gender and age groups, varied $\mathrm{CHO}$ concentrations, doses, or types in the effect measurement. The evidence found was sound enough to support the hypothesis that $\mathrm{CHO}$ solutions, when ingested during endurance exercise, have ergogenic action and a possible crossover interaction with the subject's CRF.
\end{abstract}

Keywords: endurance; performance; systematic review; scientific quality; continuous; intermittent

\section{Introduction}

Interest in the role and contribution of carbohydrates (CHOs) as an energy fuel, particularly during endurance exercise, dates back to the beginning of the 20th century [1]. Christensen and Hansen (1939) examined the role of a high-CHO diet and suggested that hypoglycemia causes fatigue during light exercise by affecting the central nervous system (CNS) [2]. In the late 1960s, it was revealed that exercise with glycogen depletion increases the resynthesis of muscle glycogen [3] and also that stored muscle glycogen plays a significant role during exercise [4]. Muscle glycogen stores are primly defined by diet prior to exercise. It has also been shown that the higher the muscle glycogen content, the higher the endurance performance [4,5]. In 1975, a study revealed that CHO feeding during prolonged exercise could increase exercise capacity, which was confirmed by another study in 1983 [5,6]. As scientific interest in the field of dietary supplements gradually grew, 
during the past 45 years, a great number of researchers have extensively investigated the effects of $\mathrm{CHO}$ consumption during endurance exercise, mostly from the perspective of determining the optimal composition and timing of $\mathrm{CHO}$ replacement beverages during exercise. Thus, the effect of $\mathrm{CHO}$ ingestion during endurance exercise has been reviewed by a great number of authors in the past and also in recent years [7-11], focusing on different areas of the $\mathrm{CHO}$ effect on performance.

It is widely accepted that endurance exercise requires a sufficient exogenous amount of $\mathrm{CHO}$ to postpone the onset of fatigue; when the $\mathrm{CHO}$ quantity is inadequate, performance is impaired $[9,10,12]$. However, many theories are still the subject of debate, and the conclusions of the relevant reviews have led to conflicting information about the optimum administration timing, dosage, type, and composition of $\mathrm{CHO}$ supplements [7-11]. For instance, four modern reviews recommend $\mathrm{CHO}$ intake up to $60 \mathrm{~g} \cdot \mathrm{h}^{-1}$ for exercise lasting up to $2.5 \mathrm{~h}$ and up to $90 \mathrm{~g} \cdot \mathrm{h}^{-1}$ when the duration of exercise exceeds $2.5 \mathrm{~h}$ [7-9]. Nevertheless, Mata et al. (2019) concluded that it is unclear which concentration $(6,8$, or $10 \%$ etc.) or dose of $\mathrm{CHO}$ solution and which $\mathrm{CHO}$ substance (maltodextrin (MD), glucose (GL), sucrose (SUC), or a combination) enhance endurance performance better [10]. They also mentioned that "attending to the existing evidence, no universal recommendations regarding $\mathrm{CHO}$ intake should suggested to athletes" [10]. In a simplified approach, Brooks (2020) states that as gastrointestinal (GI) emptying and absorption are determinant factors of exogenous glucose availability, beverages containing $4-6 \%$ GL could be efficient during exercise for euglycemia maintenance, while GL solutions $>6 \%$ are often less effective and blamed for GI discomfort [11]. It is understood, however, that the studies varied in method, exercise duration, performance assessment (e.g., capacity: time to exhaustion vs. performance: fix distance), and total quantity of $\mathrm{CHO}$ that was administered, which may explain any inconsistency in the literature.

Unexpectedly, no systematic review or meta-analysis has taken into account the possible effect of the cardio-respiratory fitness (CRF) of the tested subjects on the $\mathrm{CHO}$ intake intervention. An extensive comparative and updated review on the effect of $\mathrm{CHO}$ supplements on different types of exercise, such as cycling vs. running, also appears to be lacking. Thermal stress-induced energy metabolic changes during prolonged exercise of different modes and intensities are likely to be different [13,14]; yet, the ergogenic role of $\mathrm{CHO}$ supplementation in different ambient conditions has not been reviewed in a metaanalysis. Additionally, with only a few exceptions, most past reviews more or less failed to mention how the search for relevant studies was carried out, what the inclusion criteria were for the studies and whether they were appropriate, whether the validity of included studies was assessed, whether the methods and statistics were reliable and appropriate, how conclusions were reached, whether results were explicit, and how the studies were integrated, which can elicit overestimation or underestimation of the intervention effect [15]. No meta-analysis has examined the effect of $\mathrm{CHO}$ ingestion with no additives (e.g., caffeine, protein) during endurance exercise on endurance capacity and performance, with the use of an accurate method of combining the results of independent studies, assessing risk-of-bias, and considering the potential limitations of the eligible studies [15].

Therefore, the purpose of this study was to select relevant papers from a specific period (1975-2021) for a systematic/critical review using a meta-analytic technique. It is certain that athletes, coaches, and training instructors will be interested in seeing the actual $\mathrm{CHO}$ ergogenic supplementations' effect on different exercise modalities in association with supplement composition, concentration, administration time, and exercise duration; a systematic review conducted according to predefined methodological criteria will surely be beneficial to this audience. Therefore, the aim of this study was to investigate whether the literature supports the hypothesis that $\mathrm{CHO}$ supplementation in a liquid form during exercise enhances performance, taking into account the subjects' CRF and the relative methodological quality of the papers searched. A further aim was to establish the optimum administration time and the optimal composition and concentration of $\mathrm{CHO}$ replacement during endurance exercise, and to contribute to resolving the controversy posed by the 
previous reviewers' relevant conflicting findings. On the other hand, this study focused mainly on the scientific evidence for the efficacy of $\mathrm{CHO}$ supplementation rather than on understanding the mechanism/s involved.

\section{Materials and Methods}

\subsection{Search Strategy}

For the purposes of the present systematic review, a meta-analysis was conducted, based on the Preferred Reporting Items for Systemic Reviews and Meta-Analyses (PRISMA) and Cochrane Handbook for Systematic Reviews of Interventions statements guidelines [16,17]. Figure 1 outlines a summary of the procedures followed in this study.

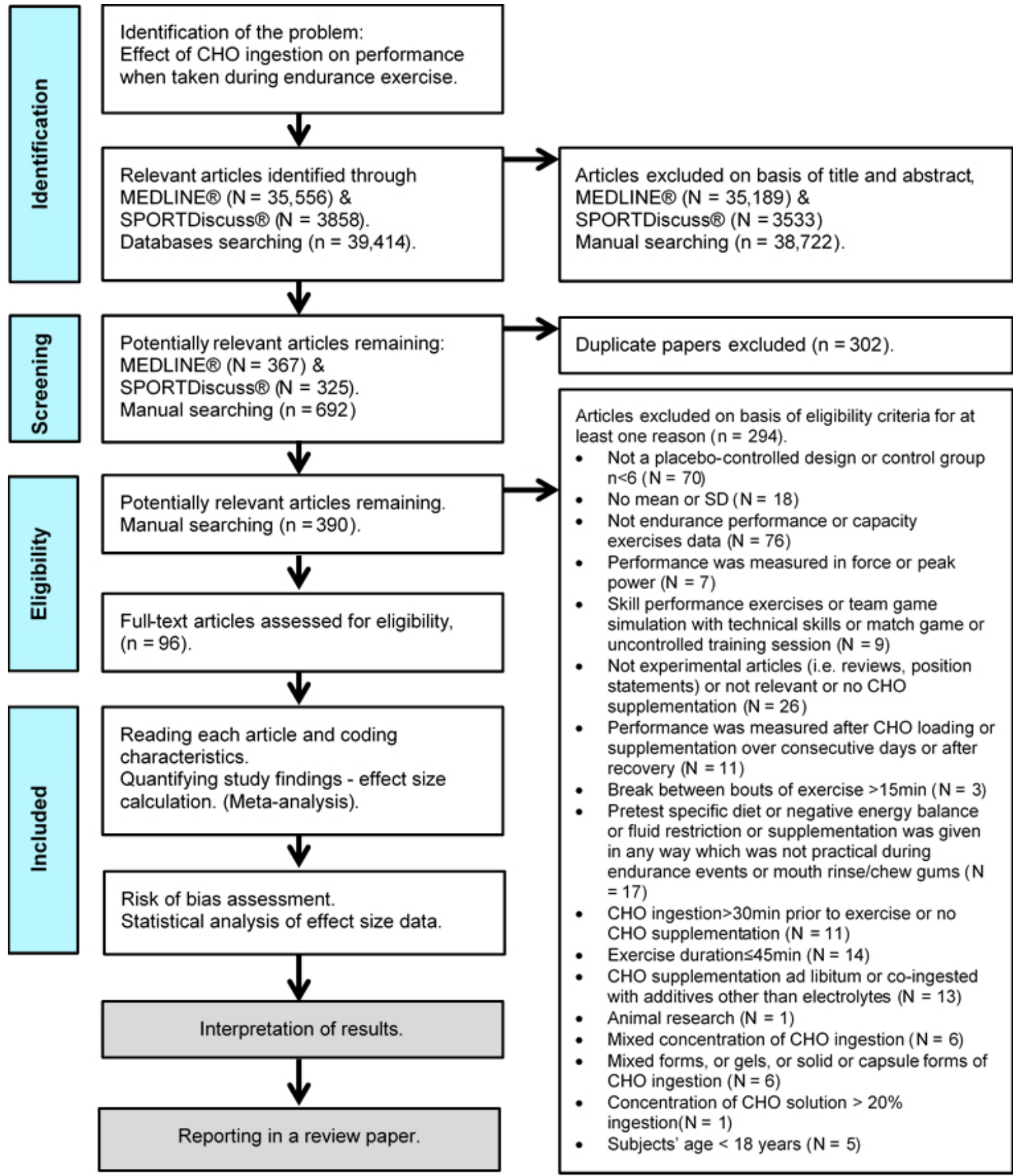

Figure 1. Prisma flowchart of the study selection process [16]. 


\subsection{Databases and Search}

The initial electronic literature review was carried out by searching the MEDLINE®(via PubMed $^{\circledR}$ ), and SPORTDiscus ${ }^{\circledR}$ (via EBSCOhost) databases until April 2021. We restricted our review to studies published since 1975, which, to our knowledge, was the year of publication of the first study showing that $\mathrm{CHO}$ feeding during prolonged exercise improves exercise capacity [5]. We did not review any study dating from August 2021 to the article submission day. We used the Boolean search syntax ((carbohydrate* OR CHO*) AND (endurance OR performance OR capacity OR exercise OR timing)). Full-text studies chosen were limited to those published in English peer-reviewed journals, with human subjects used. Additionally, control clinical trial OR/AND randomized clinical trial filters were activated, and a total of 36,605 studies from MEDLINE®and 3956 studies from SPORTDiscus ${ }^{\circledR}$ were found.

\subsection{Eligibility Criteria}

From the preliminary articles originally identified through the search of the electronic databases, all irrelevant articles were manually excluded based on their title and abstract. A number of studies were manually picked through predefined eligibility criteria by (two independent: E.Z. and G.M.) reviewers, who screened the potentially relevant papers by checking their titles, abstracts, methods, and results. In order to avoid risk of bias in selecting and rejecting papers, reviewers looked first at methods and then at the results.

The independent variables were: (a) the contents (e.g., GL, FRU) of a liquid form of supplementation only (i.e., $\mathrm{CHO} \leq 20 \%$, e.g., solution, drink, beverage); (b) the $\mathrm{CHO}$ concentration (\%) or the dose (i.e., ingestion rate $\left.\left(\mathrm{CHO} g \cdot \mathrm{h}^{-1}\right)\right)$; and (c) the timing of $\mathrm{CHO}$ supplement ingestion during an endurance exercise (at regular intervals or single bolus dose), which was not less than $30 \mathrm{~min}$ before the beginning of the event until its end. The dependent variable was defined as the effect of $\mathrm{CHO}$ supplementation on endurance performance, lasting $\geq 45 \mathrm{~min}$ (time to exhaustion or time to complete a certain distance in events of variable duration and intensity). The rationale for initially restricting attention to exercise duration effort $\geq 45$ min was that endogenous muscle glycogen is not fully depleted in 1-h all-out exercises [18]. During an exercise ( $>85 \% \dot{\mathrm{VO}}_{2}$ max) performed continuously for 20-30 min, fatigue in skeletal muscle is caused by an increased accumulation of $\mathrm{H}_{+}$[19]. Thirdly, the ergogenic influence of a CHO solution on an intense exercise of a relatively short duration may be partially explained by the solution's stimulus on the brain via mouth receptors sensitive to $\mathrm{CHO}$; thus, the ergogenic effect may be not exclusively metabolic in nature but could also be attributed to the CNS [7].

Only papers of controlled interventions, where the authors reported that they used a specific experimental method, were chosen. When an article contained more than one research arm that qualified for inclusion, they were regarded as separate 'interventions' denoted as 'trials'. In particular, studies or trials that did not involve a comparison group in a parallel or crossover design [20], single-subject design studies, studies that used sample sizes $(\mathrm{N})$ less than six subjects per group, and studies that did not provide the numerical means (not depicted) and standard deviation (SD) or standard error (SE = SD divided by the square root of the sample size) for the dependent variable were not included. Editorials, letters to the editor, government's reports, grey literature, or abstracts or scientific events or other articles indexed by non-scientific databases (not peer-reviewed) that did not contain original results were also excluded from this review. Original studies that reported the use of healthy human subjects (age $\geq 18$ years) and were relevant to the topic of interest, i.e., where appropriate, independent and dependent variables could be defined from the article's title and abstract, were included.

Additionally excluded were studies with substantial rest intervals ( $>15 \mathrm{~min}$ ), or $\mathrm{CHO}$ supplementation given during recovery from exercise, during team games, during uncontrolled training sessions, during an exercise protocol that included technical sport drills (e.g., ball drills) designed to simulate a fast-paced game, or supplementation administered in many different concentrations or given in solid (e.g., chocolate, energy bar, pudding), gel, 
capsule, or mixed forms (e.g., solution co-ingested with gel), intravenously, or in any other way, which was not practical during endurance events. Studies that used an intermittent exercise protocol designed to simulate a fast-paced game or 'stop and start' sports, using only running, jogging, and walking activities, were included. Studies that used CHO supplementation in combination with electrolytes were chosen when the control group was also provided. Studies that used CHO supplementation: ad libitum, co-ingested with additives (i.e., any neurological stimulant (e.g., caffeine), neurotransmitter or neuromodulator (e.g., taurine), $\mathrm{NAD}_{+}$precursor (e.g., nicotinic acid), substances that may have synergetic effect or are advocated in the medical literature for muscle fatigue reduction after exercise or boosting metabolism (e.g., vitamins, chromium picolinate, carnitine), and potential energetic substrate (e.g., fat, protein) [21-23]), in fasted state (except overnight fasting) or in negative energy balance, after pre-experimental $\mathrm{CHO}$ preloading or any kind of enriched or specific diet other than normal were excluded. Studies that exclusively used a fluid restriction protocol (pre supplementation or during exercise) or a protocol to investigate during exercise the effects on the hormonal response, immune response, gastric emptying, GI problems, GL oxidation, heart rate, rate of perceived exertion, cognitive performance, reaction time, resistance exercise, peak power, velocity, force, torque, energy cost or technical skills, and $\mathrm{CHO}$ mouth rinse or $\mathrm{CHO}$ chewing gum response after treatment were also excluded. Finally, after the removal of duplicate articles, 294 articles were excluded and 96 selected for further analysis (Figure 1).

\subsection{Data Extraction}

The next step was to code the characteristics and outcomes of the selected studies that were likely to influence the true intervention effect sizes [17]. Characteristics provided descriptive information about the study with the following categories: design (e.g., randomization, control/no control, statistical analysis ... ), protocol test (e.g., continuous, intermittent ... ), endurance exercise mode (e.g., cycling, running, swimming ... ), treatment variable (e.g., supplementation that was used, concentration, dose, administration time, composition, form ... ), subjects (e.g., gender, age, CRF, ... ), dependent variable (e.g., performance time, exhaustion time ... ), and environmental conditions (e.g., temperature, altitude ... ). No contact was made with the studies' authors. Two reviewers (A.K. and K.P.) independently processed data extraction from the initially selected studies. Disagreements between reviewers with regard to including or excluding data of a given study were resolved by consensus.

\subsection{Risk-of-Bias Assessment and Deficiencies in Scientific Design or Reporting}

A modified version of the Cochrane risk-of-bias assessment tool for systematic reviews was employed to assess potential risk-of-bias in the eligible studies [24]. The modification was based on empirical evidence showing that they have a biasing effect on the estimates of a treatment's effectiveness. This evidence derived from previous systematic reviews [25-28] and their importance to the reviewer in determining whether confidence should be placed on the author's conclusions based on the CONsolidated Standards of Reporting Trials 2010 guidelines [29,30]. Nine risk-of-bias items were used for all eligible studies (i.e., eligibility criteria; statistical power calculation; subject's familiarization; time series control of treatment allocation over the study period; subjects blinded to treatment; researchers blinded to treatment; reliability of measures; validity of measures; complication or dropout $\geq 15 \%$ ), which were graded as low (+), some concerns (?), and high (-) risk-ofbias. Two external researchers (K.P. and A.K.), unaware of this study's purpose and of any data that could help identify the studies' authorship (e.g., authors' names and affiliations, year, and type of publication), assessed the studies' risk-of-bias based on answers to the signaling questions independently [24,31]. Any disagreement between the two researchers was resolved by discussion; if no consensus could be reached, a third researcher (T.T.) made the final decision. 


\subsection{Statistical Analyses}

Data were analyzed using the Review Manager software, Version 5.3.4. (Cochrane Collaboration Copenhagen, The Nordic Cochrane Centre, Copenhagen, Denmark). The effect size (ES) of CHO supplementation on exercise performance was calculated as the betweensubject standardized mean difference $(\mathrm{SMD}=([$ mean post-value treatment group-mean post-value control group]/pooled variance)). Due to the nature of the test performance assessment, performance mean data were inserted into Review Manager software for analysis in a negative way (i.e., multiplied by -1 ) so that both studies (time-to-exhaustion and self-paced time-trial) corresponded to the same direction by means of the effect size in performance enhancement [32-34]. Studies with small sample sizes have a biased ES [35], and thus, each SMD was multiplied by a correction factor (g) to allow an unbiased estimate of ES [36-38]. The correction factor was calculated from the formula: $g=[1-3 /(4 \mathrm{Ni}-9)]$, where, $\mathrm{Ni}$ = pooled sample size. According to Higgins et al., (2011), SMD values of 0.2-0.4 indicate a small effect, $0.5-0.7$ indicate a medium effect, and $>0.8$ indicate a large effect [15].

Furthermore, subgroup analysis was carried out to see if they had an advantage effect on their own and also to identify aspects of any possible study heterogeneity. Depending on the related published studies' data, study trials were classified as follows: (a) with regard to the subjects' characteristics, into three gender classes (male (M), female (F), combined (MF)), four age classes (young (18-29 years), adults I (30-39 years), adults II (40-49 years) [39]), and four CRF classes based on maximal oxygen uptake data (fair, good, excellent, superior) [40]; (b) with regard to exercise task, into three exercise mode classes (cycling, running, other (triathlon, duathlon, swimming, walking, loaded marching, roller-skiing)), two exercise protocol test classes (capacity: time to exhaustion, performance: time trial), three exercise type classes (intermittent: sessions interspersed with short rest or recovery periods involve activity of lower intensity; continuous: no break between sessions, regardless of the intensity of the sessions; intermittent shuttle: sessions that simulate the activity pattern of 'stop and start' sports), and four exercise time classes ((T), (45 $\mathrm{min} \leq \mathrm{T} \leq 60 \mathrm{~min}$, $60 \mathrm{~min}<\mathrm{T} \leq 120 \mathrm{~min}, 120 \mathrm{~min}<\mathrm{T} \leq 240 \mathrm{~min}, \mathrm{~T}>240 \mathrm{~min}$ )); (c) with regard to supplementation, into seven $\mathrm{CHO}$ concentration classes $(0 \%<\mathrm{CHO} \leq 2 \%, 2 \%<\mathrm{CHO} \leq 4 \%$, $4 \%<\mathrm{CHO} \leq 6 \%, 6 \%<\mathrm{CHO} \leq 8 \%, 8 \%<\mathrm{CHO} \leq 10 \%, 10 \%<\mathrm{CHO} \leq 15 \%$, $15 \%<\mathrm{CHO} \leq 20 \%$ ), five $\mathrm{CHO}$ dose classes $\left(\mathrm{CHO}\right.$ dose $\leq 40 \mathrm{~g} \cdot \mathrm{h}^{-1}$, $40 \mathrm{~g} \cdot \mathrm{h}^{-1}<\mathrm{CHO}$ dose $\leq 60 \mathrm{~g} \cdot \mathrm{h}^{-1}, \quad 60 \mathrm{~g} \cdot \mathrm{h}^{-1}<\mathrm{CHO}$ dose $\leq 80 \mathrm{~g} \cdot \mathrm{h}^{-1}$, $80 \mathrm{~g} \cdot \mathrm{h}^{-1}<\mathrm{CHO}$ dose $\leq 100 \mathrm{~g} \cdot \mathrm{h}^{-1}$, CHO dose $>100 \mathrm{~g} \cdot \mathrm{h}^{-1}$ ), six $\mathrm{CHO}$ type classes (GL, MD, SUC, maltose (MAL), FRU, galactose (GAL)), 12 multiple transportable CHO classes ((MTC), (GL:FRU, GL:SUC, GL:MD, MD:FRU, MD:Dextrose (DEX), MD:SUC, GL:MD:FRU, GL:MD:DEX, GL:SUC:FRU, GL:MD:MAL:Saccharides, SUC:MD:IsoMAL, unclear $\mathrm{CHO}$ substances mixture), three $\mathrm{CHO}$ solution formulation classes (single-source $\mathrm{CHO}$ solution, double-source $\mathrm{CHO}$ solution, triple-or-more-source $\mathrm{CHO}$ solution), four administration time classes (prior to or at the beginning, during, prior to or at the beginning + during, late in exercise), and two supplement temperature administration classes ( $\mathrm{cool}\left(<18^{\circ} \mathrm{C}\right)$, neutral $\left(18-26^{\circ} \mathrm{C}\right)$ ); and d) with regard to ambient conditions, into three thermal condition classes $\left(\mathrm{cool}\left(<18^{\circ} \mathrm{C}\right)\right.$, neutral $\left(18-26^{\circ} \mathrm{C}\right)$, heat $\left.\left(>26^{\circ} \mathrm{C}\right)\right)$.

An assessment of the consistency of effects across eligible studies in the subgroups was also carried out. The between-study heterogeneity was assessed using the $\mathrm{I}^{2}$ statistic and the Chi-square test. According to Higgins et al. (2003) and Sedgwick (2015), values of the $\mathrm{I}^{2}$ statistic of $0-50 \%$ represent low heterogeneity, $50-74 \%$ moderate heterogeneity, and $\geq 75 \%$ high heterogeneity [41,42]. It was assumed that subgrouped studies were characterized by a high degree of homogeneity due to similar clinical and methodological aspects. Consequently, SMD could be compared to find possible differences between treatments and controlled with a fixed-effect meta-analysis model (estimating the same underlying intervention effect) by means of the inverse-variance method when $\mathrm{I}^{2}<50 \%$ [43]. However, the fixed-effects analysis may not be the proper option to account for the accessible randomeffects within the analysis. On the other hand, irrespective of the $\mathrm{I}^{2}$ statistics, the randomeffect meta-analysis model (which is usually used in case of $\mathrm{I}^{2} \geq 50 \%$ ) may provide a 
more conservative estimate that may be viewed as an 'average intervention effect' [44] and thus it was programmed to compute the pooled effect size by calculating the SMD in the current study. Nevertheless, the decision between fixed- and random-effects metaanalyses has been the subject of much debate and since many authors have argued that a fixed-effect analysis can be interpreted in the presence of heterogeneity, and that it makes fewer assumptions than a random-effects meta-analysis [45,46], Cochrane organization does not provide a universal recommendation [17]. For this reason, based on fixed-effects analysis assumptions $[45,46]$ and Cochrane organization recommendations (that it may be reasonable to present both analyses) [17], we also ran fixed-effects analysis and report these results, only in two cases, where the presence of homogeneity $\left(\mathrm{I}^{2}<50 \%\right)$ supported our actions and better interpreted our well-founded assumptions. So, the outcomes of this meta-analysis are derived from the random-effect meta-analysis model throughout the paper except otherwise stated. The presence or not of publication bias was investigated by funnel plots using the Review Manager software, Version 5.3.4. (Cochrane Collaboration Copenhagen, The Nordic Cochrane Centre, Copenhagen, Denmark) and Egger's regression analysis using the Meta-Essentials tools (Rotterdam School of Management, Erasmus University, The Netherlands).

The descriptive data of the eligible studies is presented as means. Pooled estimates of the ES derived by either subgroup or comprehensive meta-analyses are presented as SMD and $95 \%$ confidence intervals (CIs) in square brackets (SMD [95\% CI]). The alpha level for statistical significance was set at $p \leq 0.05$ a priori.

\section{Results}

\subsection{Study Characteristics}

An overview, along with the subjects' characteristics, descriptive characteristics of the protocols used, and the effect (in SMD [95\% CI]) of experimental CHO supplementation as compared to the control on an exercise task of the reviewed articles included in this meta-analysis, is reported in alphabetical order, by first author surname, in Table 1 . The comparison of experimental $\mathrm{CHO}$ supplementation vs. control on exercise outcome, including raw data (in mean and SD [95\% CI]) and risk-of-bias judgments of all trials is presented in a forest plot (Figure S1). The overview authors' judgments for each risk-of-bias item are depicted as a percentage in Figure 2.

In total, 96 studies (142 trials) involving 1560 subjects in experimental groups (1534 in control groups) satisfied the inclusion criteria and were approved for further analysis. So, 142 SMDs were assessed and the total SMD [95\% CI] estimate of $0.43[0.35,0.51]$ was significant, $(p<0.001)$. All studies used similar methods (pre-post design). The sample population of the control groups used was usually identical to that of the experimental groups in a crossover design, with the exception of three studies, which used a different sample population as control groups in a parallel design (Table 1). The participating subjects were mainly male, and their mean age was 19.3-44.0 (Table 1). Eighty-nine studies were lab-based and only seven field-based. The sport types of exercise used in the trials were cycling, running, triathlon, duathlon, roller-skiing, walking, loaded marching, swimming, and arm cranking $(100,35,2,1,1,1,1$, and 1, respectively; Table 1). 


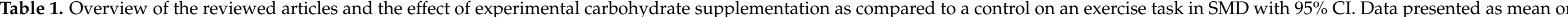

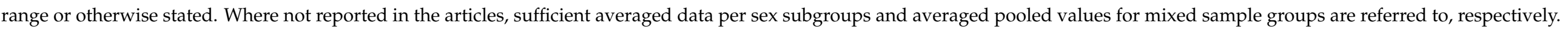

\begin{tabular}{|c|c|c|c|c|c|c|c|c|c|}
\hline Study/Trial † & Study Design & $\begin{array}{l}N / \\
\text { Gender }\end{array}$ & $\begin{array}{c}\text { Age } \\
\text { (year) }\end{array}$ & $\begin{array}{c}\text { BMI } \\
\left(\mathbf{k g} \cdot \mathrm{m}^{-2}\right)\end{array}$ & $\begin{array}{c}\dot{\mathrm{VO}_{2} \max } \\
\left(\mathrm{mL} \cdot \mathrm{kg}^{-1} \cdot \mathrm{min}^{-1}\right)\end{array}$ & $\begin{array}{l}\text { Lab/ } \\
\text { Field }\end{array}$ & $\begin{array}{c}\text { Exercise Task } \\
\text { (Brief Description) }\end{array}$ & $\begin{array}{l}\text { CHO Supplementation } \\
\text { (Brief Description) }\end{array}$ & $\begin{array}{l}\text { SMD IV, Random } \\
{[95 \% \mathrm{CI}]}\end{array}$ \\
\hline $\begin{array}{l}\text { Acker-Hewitt et al., } \\
2012 \text { [47] }\end{array}$ & CS & $10 / \mathrm{M}$ & 28.0 & 23.0 & 66.0 & $\mathrm{Lab}$ & $\begin{array}{c}20 \text { min of SS cycling [ } 60 \% \text { PPO } \\
(\text { Wmax }) \text { + a simulated } 20-\mathrm{km} \\
\text { TT, TA: } 21.5^{\circ} \mathrm{C}\end{array}$ & $\begin{array}{l}\text { 8\% solution, } 250 \mathrm{~mL} \text { fluid }(\mathrm{CHO}, \\
20 \mathrm{~g} \text { ) administered at: } \\
\text { immediately prior to exercise, } \\
\text { following the } 20 \text {-min SS, and } \\
20 \text { min into the TT }\end{array}$ & $0.09[-0.79,0.97]$ \\
\hline Alghannam 2011 [48] & CS & $6 / \mathrm{M}$ & 26.0 & 21.9 & $51.4^{*}$ & $\mathrm{Lab}$ & $\begin{array}{c}75 \text { min of intermittent } \\
\text { football-specific running } \\
\text { (interspersed with a } 15 \mathrm{~min} \\
\text { recovery) + run TF at } 80 \% \\
\dot{\mathrm{VO}_{2}} \text { peak, TA: } 20.6^{\circ} \mathrm{C}\end{array}$ & $\begin{array}{c}6.9 \% \mathrm{CHO} \text { solution }[\mathrm{MD}, \\
1 \mathrm{~g} \cdot \mathrm{kg}^{-1} \text { ] administered at: } \\
15 \mathrm{~min} \text { prior to exercise and at } \\
45 \mathrm{~min}\end{array}$ & $1.67[0.28,3.07]$ \\
\hline Ali et al., 2007 [49] & CS & $16 / \mathrm{M}$ & 21.3 & 23.0 & 56.0 & $\mathrm{Lab}$ & $\begin{array}{c}90 \text { min intermittent } \\
\text { high-intensity shuttle running } \\
\text { ( 66 sprints) protocol [LIST: } \\
15 \text { min block consists of } \\
10-12 \text { repeated cycles of } \\
\text { walking, running (at a speed } \\
\text { equivalent to } 95 \% \mathrm{~V}_{2} \text { max), } \\
\text { jogging (at a speed equivalent } \\
\text { to } 55 \% \mathrm{~V}_{2} \text { max), and } \\
\text { sprinting] }\end{array}$ & $\begin{array}{l}\text { 6.4\% CHO-E solution (Lucozade } \\
\text { Sport, GlaxoSmithKline, } \\
\text { Brentford), } 5 \mathrm{~mL} \cdot \mathrm{kg}^{-1} \text { before and } \\
2 \mathrm{~mL} \cdot \mathrm{kg}^{-1} \text { every } 15 \mathrm{~min} \text { of } \\
\text { exercise }\end{array}$ & $0.22[-0.47,0.92]$ \\
\hline $\begin{array}{l}\text { Anastasiou et al., } \\
2004 \text { i [50] }\end{array}$ & CS & $10 / \mathrm{M}$ & 25.1 & 23.0 & $56.6^{*}$ & $\mathrm{Lab}$ & $\begin{array}{l}\text { cycling time to complete the } \\
\text { target amount of work } \\
(\mathrm{J})=0.75 \times \text { Wmax } \times 3600 \\
(\sim 60 \mathrm{~min}), \mathrm{TA}: 27.9^{\circ} \mathrm{C}\end{array}$ & $\begin{array}{l}\text { GL-E drink, }\left(0.65 \mathrm{~g} \cdot \mathrm{kg}^{-1}\right) 15 \mathrm{~min} \\
\text { prior to exercise } \sim 600 \mathrm{~mL} \text { and } \\
\text { during at } 15 \text {-min intervals } \\
\sim 200 \mathrm{~mL} \text { of GL-E drink } \\
\left(0.2 \mathrm{~g} \cdot \mathrm{kg}^{-1}\right)\end{array}$ & $0.83[-0.10,1.75]$ \\
\hline $\begin{array}{l}\text { Anastasiou et al., } \\
2004 \text { ii [50] }\end{array}$ & CS & $10 / \mathrm{M}$ & 25.1 & 23.0 & $56.6^{*}$ & $\mathrm{Lab}$ & $\begin{array}{l}\text { cycling time to complete the } \\
\text { target amount of work } \\
(\mathrm{J})=0.75 \times \text { Wmax } \times 3600 \\
(\sim 60 \mathrm{~min}), \mathrm{TA}: 27.9^{\circ} \mathrm{C}\end{array}$ & $\begin{array}{c}\text { MAL-E drink, }\left(0.65 \mathrm{~g} \cdot \mathrm{kg}^{-1}\right) \\
15 \mathrm{~min} \text { prior to exercise } \sim 600 \mathrm{~mL} \\
\text { and during at } 15 \text {-min intervals } \\
\sim 200 \mathrm{~mL} \text { of MAL-E drink } \\
\left(0.2 \mathrm{~g} \cdot \mathrm{kg}^{-1}\right)\end{array}$ & $0.31[-0.57,1.20]$ \\
\hline $\begin{array}{l}\text { Anastasiou et al., } \\
2004 \text { iii [50] }\end{array}$ & CS & $10 / \mathrm{M}$ & 25.1 & 23.0 & $56.6^{*}$ & $\mathrm{Lab}$ & $\begin{array}{l}\text { cycling time to complete the } \\
\text { target amount of work } \\
(\mathrm{J})=0.75 \times \text { Wmax } \times 3600 \\
(\sim 60 \mathrm{~min}), \mathrm{TA}: 27.9^{\circ} \mathrm{C}\end{array}$ & $\begin{array}{c}\text { CHO-mix-E drink, }\left(0.65 \mathrm{~g} \cdot \mathrm{kg}^{-1}\right) \\
15 \text { min prior to exercise } \sim 600 \mathrm{~mL} \\
\text { and during at } 15-\mathrm{min} \text { intervals } \\
\sim 200 \mathrm{~mL} \text { of CHO-mix-E drink } \\
\left(0.2 \mathrm{~g} \cdot \mathrm{kg}^{-1}\right)\end{array}$ & $1.01[0.07,1.96]$ \\
\hline
\end{tabular}


Table 1. Cont

\begin{tabular}{|c|c|c|c|c|c|c|c|c|c|}
\hline Study/Trial + & Study Design & $\begin{array}{c}N / \\
\text { Gender }\end{array}$ & $\begin{array}{c}\text { Age } \\
\text { (year) }\end{array}$ & $\begin{array}{c}\text { BMI } \\
\left(\mathbf{k g} \cdot \mathrm{m}^{-2}\right)\end{array}$ & $\begin{array}{c}\dot{\mathrm{VO}}{ }_{2} \max \\
\left(\mathrm{mL} \cdot \mathrm{kg}^{-1} \cdot \mathrm{min}^{-1}\right)\end{array}$ & $\begin{array}{l}\text { Lab/ } \\
\text { Field }\end{array}$ & $\begin{array}{c}\text { Exercise Task } \\
\text { (Brief Description) }\end{array}$ & $\begin{array}{l}\text { CHO Supplementation } \\
\text { (Brief Description) }\end{array}$ & $\begin{array}{l}\text { SMD IV, Random } \\
{[95 \% \text { CI }]}\end{array}$ \\
\hline Angus et al., 2000 [51] & CS & $8 / \mathrm{M}$ & 22.0 & 23.0 & $65.4 *$ & Lab & $\begin{array}{l}\text { cycling time to complete "as } \\
\text { quickly as possible" } 35 \mathrm{~kJ} \cdot \mathrm{kg}^{-1} \text {, } \\
\text { TA: } 20.0-22.0^{\circ} \mathrm{C}\end{array}$ & $\begin{array}{c}\text { 6\% CHO solution (Gatorade, } \\
\text { Quaker Oats Co.), } 250 \mathrm{~mL} \text { at } \\
15 \text {-min intervals }\end{array}$ & $1.23[0.13,2.33]$ \\
\hline Bailey et al., 2000 i [52] & CS & $9 / \mathrm{F}$ & 27.0 & 21.5 & $49.6^{*}$ & Lab & $\begin{array}{l}\mathrm{TE} \text { cycling at } 70 \% \mathrm{VO}_{2} \text { peak, } \\
\text { during follicular phase of the } \\
\text { menstrual cycle, TA: } 22.7^{\circ} \mathrm{C}\end{array}$ & $\begin{array}{c}\text { 6\% CHO solution (Gatorade, } \\
\text { Quaker Oats Co.), } \mathrm{CHO} \\
0.6 \mathrm{~g} \cdot \mathrm{kg}^{-1} \cdot \mathrm{h}^{-1},\left(5 \mathrm{~mL} \cdot \mathrm{kg}^{-1}\right. \\
\text { every } 30 \mathrm{~min} \text { beginning at min } \\
30 \text { of exercise) }\end{array}$ & $0.57[-0.38,1.52]$ \\
\hline Bailey et al., 2000 ii [52] & CS & $9 / \mathrm{F}$ & 27.0 & 21.5 & $49.6^{*}$ & Lab & $\begin{array}{l}\text { TE cycling at } 70 \% \mathrm{VO}_{2} \text { peak, } \\
\text { during luteal phase of the } \\
\text { menstrual cycle, TA: } 22.7^{\circ} \mathrm{C}\end{array}$ & $\begin{array}{c}\text { 6\% CHO solution (Gatorade, } \\
\text { Quaker Oats Co.), } \mathrm{CHO} \\
0.6 \mathrm{~g} \cdot \mathrm{kg}^{-1} \cdot \mathrm{h}^{-1},\left(5 \mathrm{~mL} \cdot \mathrm{kg}^{-1}\right. \\
\text { every } 30 \mathrm{~min} \text { beginning at min } \\
30 \text { of exercise) }\end{array}$ & $0.34[-0.59,1.27]$ \\
\hline Baldassarre et al., 2021 [53] & CS & $\begin{array}{l}8 / 5 \mathrm{M} \\
3 \mathrm{~F}\end{array}$ & 23.0 & 23.0 & $\#$ & $\mathrm{Lab}$ & $\begin{array}{c}3 \times 30 \mathrm{~min}(20 \mathrm{~s} \text { interval) } \\
\text { swimming at a pre-set pace } \\
\text { (corresponding to } 10-\mathrm{km})+\mathrm{a} \\
\mathrm{TE} \text { at } 100 \% \mathrm{VO}_{2} \text { peak, } \\
\mathrm{TA}: 27^{\circ} \mathrm{C}\end{array}$ & $\begin{array}{l}\text { 8\% CHO solution, } 45 \mathrm{~g} \text { GL:FRU } \\
\text { in ratio of } 1: 1 \text { in } 550 \mathrm{~mL} \text { of water } \\
\text { (Enervitene Sport Cheerpack, } \\
\text { Enervit@) during each of the two } \\
\text { intervals (total } 60 \mathrm{~g} \cdot \mathrm{h}^{-1} \text { of CHO) }\end{array}$ & $0.83[-0.21,1.86]$ \\
\hline Baur et al., 2014 ii [54] & CS & $8 / \mathrm{M}$ & 25.0 & 23.8 & 62.0 & $\mathrm{Lab}$ & $\begin{array}{l}120 \text { min of constant-load } \\
\text { cycling at } 55 \% \text { Wmax }+ \text { a } \\
\text { simulated } 30 \mathrm{~km} \mathrm{TT}\end{array}$ & $\begin{array}{l}8 \% \text { CHO-E beverage } \\
\text { (moderate-GL beverage), } 600 \mathrm{~mL} \\
\text { prior to exercise, } 150 \mathrm{~mL} \text { bolus } \\
\text { every } 15 \mathrm{~min} \text { during the } \\
\text { constant-load part of the trial } \\
\text { (total: } 1200 \mathrm{~mL} \text { ) and at three } \\
\text { points during the } 30-\mathrm{km} \text { TT }(7.5, \\
15 \text {, and } 22.5 \mathrm{~km} \text {; total: } 450 \mathrm{~mL} \text { ) }\end{array}$ & $0.55[-0.46,1.55]$ \\
\hline
\end{tabular}


Table 1. Cont.

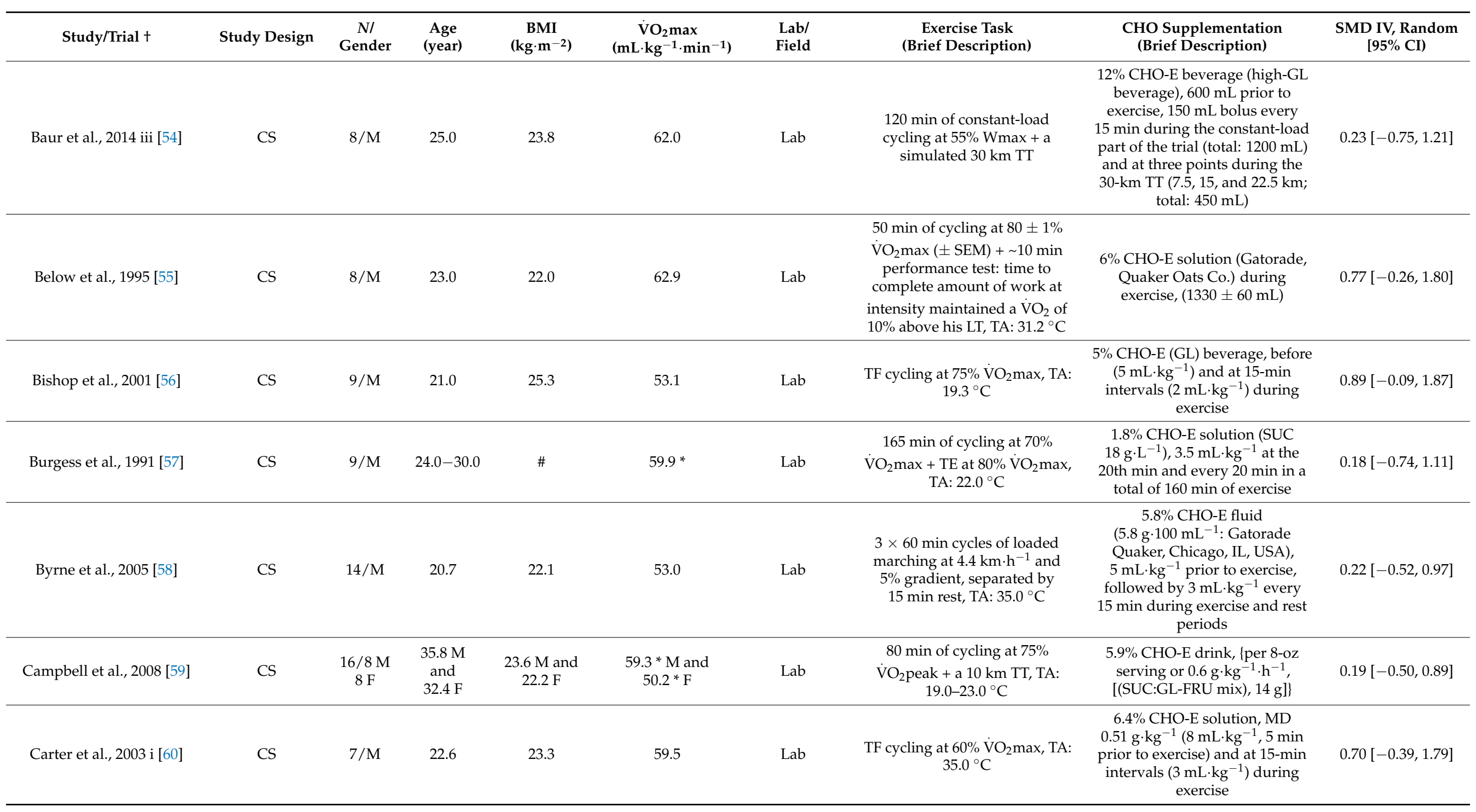


Table 1. Cont.

\begin{tabular}{|c|c|c|c|c|c|c|c|c|c|}
\hline Study/Trial + & Study Design & $\begin{array}{c}N / \\
\text { Gender }\end{array}$ & $\begin{array}{l}\text { Age } \\
\text { (year) }\end{array}$ & $\begin{array}{c}\text { BMI } \\
\left(\mathbf{k g} \cdot \mathbf{m}^{-2}\right)\end{array}$ & $\begin{array}{c}\dot{\mathrm{V} \mathrm{O}_{2} \mathrm{max}} \\
\left(\mathrm{mL} \cdot \mathrm{kg}^{-1} \cdot \mathrm{min}^{-1}\right)\end{array}$ & $\begin{array}{l}\text { Lab/ } \\
\text { Field }\end{array}$ & $\begin{array}{c}\text { Exercise Task } \\
\text { (Brief Description) }\end{array}$ & $\begin{array}{l}\text { CHO Supplementation } \\
\text { (Brief Description) }\end{array}$ & $\begin{array}{l}\text { SMD IV, Random } \\
{[95 \% \mathrm{CI}]}\end{array}$ \\
\hline Carter et al., 2003 ii [60] & CS & $8 / \mathrm{M}$ & 22.6 & 23.3 & 59.5 & $\mathrm{Lab}$ & $\begin{array}{c}\text { TF cycling at } 73 \% \mathrm{~V}_{2} \text { max, TA: } \\
35.0^{\circ} \mathrm{C}\end{array}$ & $\begin{array}{c}6.4 \% \mathrm{CHO}-\mathrm{E} \text { solution, } \mathrm{MD} \\
0.51 \mathrm{~g} \cdot \mathrm{kg}^{-1}\left(8 \mathrm{~mL} \cdot \mathrm{kg}^{-1}, 5 \mathrm{~min}\right. \\
\text { prior to exercise) and at } 15-\mathrm{min} \\
\text { intervals }\left(3 \mathrm{~mL} \cdot \mathrm{kg}^{-1}\right) \text { during } \\
\text { exercise }\end{array}$ & $0.38[-0.61,1.37]$ \\
\hline Carter et al., 2005 i [61] & CS & $8 / \mathrm{M}$ & 24.0 & 23.2 & 60.5 & $\mathrm{Lab}$ & $\begin{array}{c}\text { TE cycling at } 60 \% \mathrm{~V}_{2} \max \text {, TA: } \\
35.0^{\circ} \mathrm{C}\end{array}$ & $\begin{array}{l}6.4 \% \text { CHO solution (sweetened, } \\
\mathrm{MD}), 8 \mathrm{~mL} \cdot \mathrm{kg}^{-1} 5 \mathrm{~min} \text { prior to } \\
\text { exercise and at } 15 \text {-min intervals } \\
3 \mathrm{~mL} \cdot \mathrm{kg}^{-1} \text { during exercise }\end{array}$ & $0.49[-0.51,1.49]$ \\
\hline Carter et al., 2005 ii [61] & CS & $8 / \mathrm{M}$ & 24.0 & 23.2 & 60.5 & $\mathrm{Lab}$ & $\begin{array}{c}\text { TE cycling at } 60 \% \dot{\mathrm{VO}}_{2} \text { max, TA: } \\
35.0^{\circ} \mathrm{C}\end{array}$ & $\begin{array}{c}6.4 \% \text { CHO solution } \\
\text { (non-sweetened, MD), } \\
8 \mathrm{~mL} \cdot \mathrm{kg}^{-1} 5 \text { min prior to } \\
\text { exercise and at } 15 \text {-min intervals } \\
3 \mathrm{~mL} \cdot \mathrm{kg}^{-1} \text { during exercise }\end{array}$ & $0.44[-0.56,1.43]$ \\
\hline Clark et al., 2000 [62] & PS & $\begin{array}{l}\text { 7/\#M } \\
\text { \#F }\end{array}$ & $23.0-26.0$ & 24.3 & $64.0 *$ & $\mathrm{Lab}$ & km cycling TT & $\begin{array}{l}7.6 \% \mathrm{CHO} \text { solution } \\
\text { (GL-polymer), } 8 \mathrm{~mL} \cdot \mathrm{kg}^{-1} 30 \mathrm{~min} \\
\text { before, } 2 \mathrm{~mL} \cdot \mathrm{kg}^{-1} 2 \mathrm{~min} \text { before } \\
\text { and at } 10,20, \text { and } 30 \mathrm{~km} \text { of the TT }\end{array}$ & $0.00[-1.05,1.05]$ \\
\hline Cole et al., 1993 i [64] & CS & $10 / \mathrm{M}$ & 28.0 & 24.0 & 59.6 & $\mathrm{Lab}$ & $\begin{array}{c}105 \text { min of cycling at } 70 \% \\
\mathrm{VO}_{2} \text { max }+15 \text { min all out ride } \\
\text { performance, TA: } 23.1^{\circ} \mathrm{C}\end{array}$ & $\begin{array}{c}\text { 6\% CHO-E solution (G:SUC) at } \\
15-\mathrm{min} \text { intervals } \\
\left(9.75 \mathrm{~mL} \cdot \mathrm{kg}^{-1} \cdot \mathrm{h}^{-1}\right)\end{array}$ & $0.23[-0.65,1.11]$ \\
\hline Cole et al., 1993 ii [64] & CS & $10 / \mathrm{M}$ & 28.0 & 24.0 & 59.6 & $\mathrm{Lab}$ & $\begin{array}{c}5 \text { min of cycling at } 70 \% \\
\mathrm{VO}_{2} \text { max }+15 \text { min all out ride } \\
\text { performance, TA: } 23.1{ }^{\circ} \mathrm{C}\end{array}$ & $\begin{array}{l}\text { 8.3\% CHO-E syrup (high FRU } \\
\text { corn) at } 15 \text {-min intervals } \\
\left(9.75 \mathrm{~mL} \cdot \mathrm{kg}^{-1} \cdot \mathrm{h}^{-1}\right)\end{array}$ & $0.22[-0.66,1.10]$ \\
\hline Cole et al., 1993 iii [64] & CS & $10 / \mathrm{M}$ & 28.0 & 24.0 & 59.6 & $\mathrm{Lab}$ & $\begin{array}{c}105 \text { min of cycling at } 70 \% \\
\mathrm{VO}_{2} \text { max }+15 \text { min all out ride } \\
\text { performance, TA: } 23.1^{\circ} \mathrm{C}\end{array}$ & $\begin{array}{l}\text { 8.3\% CHO-E solution (6\% high } \\
\text { FRU corn syrup }+2.3 \% \\
\text { GL-polymer) at } 15-\text { min intervals } \\
\left(9.75 \mathrm{~mL} \cdot \mathrm{kg}^{-1} \cdot \mathrm{h}^{-1}\right)\end{array}$ & $0.21[-0.67,1.09]$ \\
\hline
\end{tabular}


Table 1. Cont

\begin{tabular}{|c|c|c|c|c|c|c|c|c|c|}
\hline Study/Trial † & Study Design & $\begin{array}{c}N / \\
\text { Gender }\end{array}$ & $\begin{array}{c}\text { Age } \\
\text { (year) }\end{array}$ & $\begin{array}{c}\text { BMI } \\
\left(\mathrm{kg} \cdot \mathrm{m}^{-2}\right)\end{array}$ & $\begin{array}{c}\dot{\mathrm{VO}_{2} \max } \\
\left(\mathrm{mL} \cdot \mathrm{kg}^{-1} \cdot \mathrm{min}^{-1}\right)\end{array}$ & $\begin{array}{l}\text { Lab/ } \\
\text { Field }\end{array}$ & $\begin{array}{c}\text { Exercise Task } \\
\text { (Brief Description) }\end{array}$ & $\begin{array}{l}\text { CHO Supplementation } \\
\text { (Brief Description) }\end{array}$ & $\begin{array}{l}\text { SMD IV, Random } \\
{[95 \% \text { CI) }}\end{array}$ \\
\hline Cole et al., 2018 [65] & CS & $14 / \mathrm{M}$ & 42.6 & 23.7 & 57.6 & Lab & $\begin{array}{c}120 \text { min of cycling at a } \\
\text { submaximal exercise intensity } \\
(60 \% \text { Maximal Minute Power), } \\
\text { TA: } 19.6{ }^{\circ} \mathrm{C}\end{array}$ & $\begin{array}{c}\text { 6\% CHO solution (MD } \\
18 \mathrm{~g} \cdot 300 \mathrm{~mL}^{-1} \text { of water: } \\
\text { Blackburn Distributions, } \\
\text { Blackburn, UK) every } 30 \mathrm{~min} \\
\text { during exercise }\end{array}$ & $3.96[2.62,5.30]$ \\
\hline Coletta et al., 2013 i [66] & CS & $12 / \mathrm{M}$ & $18.0-55.0$ & 22.7 & 59.7 & Field & $\begin{array}{l}19.2 \mathrm{~km} \text { run at a race pace }+ \\
1.92 \mathrm{~km} \text { sprint to the finish }\end{array}$ & $\begin{array}{l}6 \% \mathrm{CHO} \text { (Gatorade, Inc., } \\
\text { Chicago, IL, USA) of } 120 \mathrm{~mL} \\
\text { servings } 5 \mathrm{~min} \text { before the start, } \\
\text { and every } 4 \mathrm{~km} \text { throughout the } \\
\text { run (total: } 600 \mathrm{~mL} \text { ) }\end{array}$ & $0.08[-0.72,0.88]$ \\
\hline Coletta et al., 2013 ii [66] & CS & $12 / \mathrm{M}$ & $18.0-55.0$ & 22.7 & 59.7 & Field & $\begin{array}{l}19.2 \mathrm{~km} \text { run at a race pace }+ \\
1.92 \mathrm{~km} \text { sprint to the finish }\end{array}$ & $\begin{array}{l}7.4 \% \text { CHO (Gatorade, Inc., } \\
\text { Chicago, IL, USA) of } 120 \mathrm{~mL} \\
\text { servings } 5 \text { min before the start, } \\
\text { and every } 4 \mathrm{~km} \text { throughout the } \\
\text { run (total: } 600 \mathrm{~mL} \text { ) }\end{array}$ & $-0.07[-0.87,0.73]$ \\
\hline Cureton et al., 2007 [67] & CS & $16 / \mathrm{M}$ & 27.5 & 23.2 & 60.5 & Lab & $\begin{array}{c}120 \text { min of cycling at intensity } \\
\text { between } 60 \% \text { and } 75 \% \\
\mathrm{VO}_{2} \text { max every } 15 \mathrm{~min}+ \\
15 \text { min all out ride } \\
\text { performance, TA: } 28.5^{\circ} \mathrm{C}\end{array}$ & $\begin{array}{l}\text { 6\% CHO-E fluid (Gatorade®, } \\
\text { Quaker Oats Co., Barrington, IL, } \\
\text { USA), } 6 \mathrm{~mL} \cdot \mathrm{kg}^{-1} \text { ( } 10 \text { min before } \\
\text { and immediately) prior to } \\
\text { exercise and } 3 \mathrm{~mL} \cdot \mathrm{kg}^{-1} \text { every } \\
15 \mathrm{~min} \text { intervals over the first } \\
105 \mathrm{~min} \text { of exercise }\end{array}$ & $0.35[-0.35,1.05]$ \\
\hline Currell et al., 2008 i [68] & CS & $8 / \mathrm{M}$ & 32.0 & $\#$ & 64.7 & Lab & $\begin{array}{c}120 \mathrm{~min} \text { of cycling exercise at } \\
55 \% \mathrm{Wmax}+\mathrm{a} \text { TT to complete } \\
\text { a set amount of work as } \\
\text { quickly as possible }(\sim 60 \mathrm{~min}), \\
\text { TA: } 20.0-23.0^{\circ} \mathrm{C}\end{array}$ & $\begin{array}{c}14.4 \% \text { GL beverage }\left(1.8 \mathrm{~g} \cdot \mathrm{min}^{-1}\right) \\
600 \mathrm{~mL} \text { prior to exercise and } \\
150 \mathrm{~mL} \text { every } 15 \mathrm{~min} \text { throughout } \\
\text { the SS period and at } 25,50 \text {, and } \\
75 \% \text { of the TT }\end{array}$ & $2.55[1.14,3.97]$ \\
\hline Currell et al., 2008 ii [68] & CS & $8 / \mathrm{M}$ & 32.0 & $\#$ & 64.7 & $\mathrm{Lab}$ & $\begin{array}{c}120 \mathrm{~min} \text { of cycling exercise at } \\
55 \% \text { Wmax }+ \text { a TT to complete } \\
\text { a set amount of work as } \\
\text { quickly as possible }(\sim 60 \mathrm{~min}), \\
\text { TA: } 20.0-23.0^{\circ} \mathrm{C}\end{array}$ & $\begin{array}{l}14.4 \% \text { GL:FRU beverage in a } \\
\text { ratio of } 2: 1\left(1.8 \mathrm{~g} \cdot \mathrm{min}^{-1}\right), 600 \mathrm{~mL} \\
\text { prior to exercise and } 150 \mathrm{~mL} \\
\text { every } 15 \mathrm{~min} \text { throughout the SS } \\
\text { period and at } 25,50 \text {, and } 75 \% \text { of } \\
\text { the TT }\end{array}$ & $4.37[2.37,6.37]$ \\
\hline Davis et al., 2000 [69] & CS & $8 / \mathrm{M}$ & 27.1 & $\#$ & $55.0 *$ & Lab & $\begin{array}{l}10 \text { min warm up, } 5 \times 15 \text { min } \\
\text { bouts of intermittent shuttle } \\
\text { running (at } 95 \text { and } 55 \% \text { of } \\
\dot{\mathrm{VO}}_{2} \text { max separated by } 3 \mathrm{~min} \\
\text { rest) }+1 \text { bout of intermittent } \\
\text { shuttle running to fatigue }\end{array}$ & $\begin{array}{l}\text { 6\% CHO-E drink }\left(5 \mathrm{~mL} \cdot \mathrm{kg}^{-1}\right. \\
\left.\mathrm{CHO} 60 \mathrm{~g} \cdot \mathrm{L}^{-1}\right) 10 \mathrm{~min} \text { prior to } \\
\text { exercise and at } 15 \text {-min intervals }\end{array}$ & $0.49[-0.51,1.49]$ \\
\hline
\end{tabular}


Table 1. Cont.

\begin{tabular}{|c|c|c|c|c|c|c|c|c|c|}
\hline Study/Trial † & Study Design & $\begin{array}{c}N / \\
\text { Gender }\end{array}$ & $\begin{array}{l}\text { Age } \\
\text { (year) }\end{array}$ & $\begin{array}{c}\text { BMI } \\
\left(\mathbf{k g} \cdot \mathrm{m}^{-2}\right)\end{array}$ & $\begin{array}{c}\dot{\mathrm{VO}} \mathrm{O}_{2} \mathrm{max} \\
\left(\mathrm{mL} \cdot \mathrm{kg}^{-1} \cdot \mathrm{min}^{-1}\right)\end{array}$ & $\begin{array}{l}\text { Lab/ } \\
\text { Field }\end{array}$ & $\begin{array}{c}\text { Exercise Task } \\
\text { (Brief Description) }\end{array}$ & $\begin{array}{l}\text { CHO Supplementation } \\
\text { (Brief Description) }\end{array}$ & $\begin{array}{l}\text { SMD IV, Random } \\
{[95 \% \text { CI }]}\end{array}$ \\
\hline Desbrow et al., 2004 [70] & CS & $9 / \mathrm{M}$ & 30.0 & $\#$ & $65.1 *$ & $\mathrm{Lab}$ & $\begin{array}{c}\text { amount of cycling work } \\
\left(14 \mathrm{~kJ} \cdot \mathrm{kg}^{-1}\right) \text { as fast as possible } \\
\text { (equal to } \sim 60 \mathrm{~min} \text { at } \sim 75 \% \\
\text { Wmax), TA: } 22.0^{\circ} \mathrm{C}\end{array}$ & $\begin{array}{l}6 \% \text { CHO-E drink (Gatorade, } \\
\text { Quaker Oats Co.), } 8 \mathrm{~mL} \cdot \mathrm{kg}^{-1} \\
\text { prior to exercise and } 2 \mathrm{~mL} \cdot \mathrm{kg}^{-1} \\
\text { between } 20-30 \%, 50-60 \% \text {, } \\
70-80 \% \text { of the total amount of } \\
\text { work }\end{array}$ & $0.02[-0.90,0.95]$ \\
\hline Duhamel et al., 2007 [71] & CS & $\begin{array}{l}15 / 14 \mathrm{M} \\
1 \mathrm{~F}\end{array}$ & 19.3 & 24.5 & $44.0 *$ & $\mathrm{Lab}$ & $\begin{array}{c}\text { TF cycling at } \sim 60 \% \dot{\mathrm{VO}}_{2} \text { peak, } \\
\text { TA: } 20.0{ }^{\circ} \mathrm{C}\end{array}$ & $\begin{array}{c}6 \% \mathrm{CHO}-\mathrm{E} \text { solution } \\
\left(\mathrm{CHO}>1 \mathrm{~g} \cdot \mathrm{kg}^{-1}, 100-300 \mathrm{~mL}\right) \\
\text { after } 30 \mathrm{~min} \text { of exercise and every } \\
15 \mathrm{~min} \text { thereafter, served at } \\
20.0^{\circ} \mathrm{C}\end{array}$ & $0.85[0.10,1.60]$ \\
\hline El-Sayed et al., 1995 [72] & CS & $9 / \mathrm{M}$ & 23.8 & 22.2 & 60.7 & $\mathrm{Lab}$ & $\begin{array}{l}60 \text { min of continuous cycling at } \\
\text { the external workload } \\
\text { predicted to elicit } 70 \% \mathrm{VO}_{2} \max \\
\text { + a } 10 \text { min self-paced, all-out } \\
\text { effort performance ride, TA: } \\
22.0^{\circ} \mathrm{C}\end{array}$ & $\begin{array}{l}7.5 \% \mathrm{GL} \text { solution }\left(3 \mathrm{~mL} \cdot \mathrm{kg}^{-1}\right) \text { at } \\
15 \mathrm{~min} \text { prior to exercise and at } \\
20 \text {-min intervals }\left(3 \mathrm{~mL} \cdot \mathrm{kg}^{-1}\right) \\
\text { during the submaximal exercise }\end{array}$ & $0.57[-0.38,1.52]$ \\
\hline El-Sayed et al., 1997 [73] & CS & $8 / \mathrm{M}$ & 24.0 & 21.9 & $66.5^{*}$ & $\mathrm{Lab}$ & $\begin{array}{c}60 \text { min of simulated cycling TT } \\
\text { at a self-selected maximal pace, } \\
\text { TA: } 22.0^{\circ} \mathrm{C}\end{array}$ & $\begin{array}{l}8 \% \text { GL solution }\left(4.5 \mathrm{~mL} \cdot \mathrm{kg}^{-1}\right) \\
\text { prior to exercise }\end{array}$ & $0.16[-0.82,1.14]$ \\
\hline Finger et al., 2018 [74] & CS & $13 / \mathrm{M}$ & 29.7 & 23.1 & 62.2 & Field & $\begin{array}{c}\text { running } 10 \mathrm{~km}+\text { cycling } 40 \mathrm{~km} \\
+5 \mathrm{~km} \text { running (TT } 5 \mathrm{~km} \text { ) at a } \\
\text { self-selected pace, TA: } \\
18.0-22.0^{\circ} \mathrm{C}\end{array}$ & $\begin{array}{l}75 \mathrm{~g} \text { of MD diluted in } 450 \mathrm{~mL} \text { of } \\
\text { cold water, doses of } 150 \mathrm{~mL} \text { at } \\
\text { kilometers } 5,20 \text {, and } 35 \text { of the } \\
\text { cycling section }\end{array}$ & $0.04[-0.73,0.81]$ \\
\hline Fulco et al., 2007 i [75] & PS & $\begin{array}{l}9 / \# \mathrm{M} \\
\text { \#F }\end{array}$ & $30.0-30.7$ & 23.5 & $43.4^{*}$ & $\mathrm{Lab}$ & $\begin{array}{c}\text { amount of cycling work } \\
\text { (720 kJ) as fast as possible } \\
(\sim 60 \text { min of cycling at } 4300 \mathrm{~m} \\
\text { altitude while living at the } \\
\text { same altitude for } 1 \text { days and } \\
\text { acclimatization } \sim 2 \text { years at } \\
2000 \mathrm{~m})\end{array}$ & $\begin{array}{c}10 \% \text { CHO solution } \\
\text { [(mass / volume, } 9 \%) \mathrm{MD}+2 \% \\
\text { GL + 1\% FRU: Ergo Drink. U.S. } \\
\text { Army Soldier Systems } \\
\text { Command, Natick, MA)] at the } \\
\text { start of the exercise every } 15 \text { min } \\
\text { thereafter }\left(0.175 \mathrm{~g}^{-1} \mathrm{~kg}^{-1}\right)\end{array}$ & $0.58[-0.48,1.64]$ \\
\hline Fulco et al., 2007 ii [75] & PS & $\begin{array}{l}9 / \# \mathrm{M} \\
\text { \#F }\end{array}$ & $30.0-30.7$ & 23.5 & $43.4^{*}$ & $\mathrm{Lab}$ & $\begin{array}{l}\text { amount of cycling work } \\
\text { (720 kJ) as fast as possible } \\
(\sim 60 \text { min of cycling at } 4300 \mathrm{~m} \\
\text { altitude while living at the } \\
\text { same altitude for } 3 \text { days } \\
\text { acclimatization } \sim 2 \text { years at } \\
2000 \mathrm{~m})\end{array}$ & $\begin{array}{c}10 \% \text { CHO solution } \\
\text { [(mass/volume, } 9 \%) \text { MD + } 2 \% \\
\text { GL + 1\% FRU: Ergo Drink. U.S. } \\
\text { Army Soldier Systems Comm, } \\
\text { Natick, MA, USA }) \text { at the start of } \\
\text { the exercise every } 15 \mathrm{~min} \\
\text { thereafter }\left(0.175 \mathrm{~g} \cdot \mathrm{kg}^{-1}\right)\end{array}$ & $0.43[-0.62,1.48]$ \\
\hline
\end{tabular}


Table 1. Cont.

\begin{tabular}{|c|c|c|c|c|c|c|c|c|c|}
\hline Study/Trial † & Study Design & $\begin{array}{c}N / \\
\text { Gender }\end{array}$ & $\begin{array}{c}\text { Age } \\
\text { (year) }\end{array}$ & $\begin{array}{c}\text { BMI } \\
\left(\mathbf{k g} \cdot \mathbf{m}^{-2}\right)\end{array}$ & $\begin{array}{c}\dot{\mathrm{V} \mathrm{O}_{2} \max } \\
\left(\mathrm{mL} \cdot \mathrm{kg}^{-1} \cdot \mathrm{min}^{-1}\right)\end{array}$ & $\begin{array}{l}\text { Lab/ } \\
\text { Field }\end{array}$ & $\begin{array}{c}\text { Exercise Task } \\
\text { (Brief Description) }\end{array}$ & $\begin{array}{l}\text { CHO Supplementation } \\
\text { (Brief Description) }\end{array}$ & $\begin{array}{l}\text { SMD IV, Random } \\
{[95 \% \text { CI })}\end{array}$ \\
\hline Funnell et al., 2017 i [76] & CS & $16 / \mathrm{M}$ & 23.0 & 23.5 & 54.2 & Lab & $\begin{array}{l}3 \text { blocks of the LIST (simulated } \\
\text { soccer performance, totaling } \\
45 \mathrm{~min} \text { ), each block ( } 15 \mathrm{~min}) \\
\text { consisted of } \sim 11 \text { repeated } \\
\text { cycles of walking (three } \\
\left.\text { shuttles at } 1.5 \mathrm{~m} \cdot \mathrm{s}^{-1}\right) \text {, sprinting } \\
(15 \mathrm{~m}) \text {, rest }(4 \mathrm{~s}), \text { jogging (three } \\
\text { shuttles at } 55 \% \text { predicted } \\
\dot{\mathrm{VO}}_{2} \text { max) cruising (three } \\
\text { shuttles at } 95 \% \text { predicted } \\
\dot{\mathrm{VO}}_{2} \text { max), } 3 \mathrm{rd} \text { block was } \\
\text { "self-selected" intensity } \\
\text { distance was recorded }\end{array}$ & $\begin{array}{l}\text { 12\% CHO-E solution } \\
\text { (SUC:MD:IsoMAL), } 250 \mathrm{~mL} \\
\text { before the LIST }\end{array}$ & $-0.28[-0.97,0.42]$ \\
\hline Funnell et al., 2017 ii [76] & CS & $16 / \mathrm{M}$ & 23.0 & 23.5 & 54.2 & Lab & $\begin{array}{l}6 \text { blocks of the LIST (simulated } \\
\text { soccer performance, totaling } \\
90 \text { min), each block (15 min) } \\
\text { consisted of } \sim 11 \text { repeated } \\
\text { cycles of walking (three } \\
\left.\text { shuttles at } 1.5 \mathrm{~m} \cdot \mathrm{s}^{-1}\right) \text {, sprinting } \\
(15 \mathrm{~m}) \text {, rest }(4 \mathrm{~s}), \text { jogging (three } \\
\text { shuttles at } 55 \% \text { predicted } \\
\dot{\mathrm{V}} \mathrm{O}_{2} \text { max) cruising (three } \\
\text { shuttles at } 95 \% \text { predicted } \\
\dot{\mathrm{V}} \mathrm{O}_{2} \text { max), sixth block was } \\
\text { "self-selected" intensity } \\
\text { distance was recorded }\end{array}$ & $\begin{array}{c}12 \% \text { CHO-E solution } \\
\text { (SUC:MD:IsoMAL), } 250 \mathrm{~mL} \\
\text { before at half-time of the LIST }\end{array}$ & $-0.23[-0.92,0.47]$ \\
\hline Ganio et al., 2010 [77] & CS & $14 / \mathrm{M}$ & 27.0 & 22.8 & 60.4 & $\mathrm{Lab}$ & $\begin{array}{c}120 \text { min of submaximal cycling } \\
\text { (alternating } 61 \pm 5 \% 75 \pm 5 \% \\
\left.\dot{\mathrm{VO}}_{2} \max \right)+ \text { a } 15 \text {-min maximal } \\
\text { effort cycling (pedal } \\
\text { revolutions increased linearly), } \\
\text { TA: } 28.7^{\circ} \mathrm{C}\end{array}$ & $\begin{array}{c}\text { 6\% CHO-E solution (Gatorade, } \\
\text { Quaker Oats Co., Barrington, IL, } \\
\text { USA) before }\left(6 \mathrm{~mL} \cdot \mathrm{kg}^{-1}\right) \text { every } \\
15 \mathrm{~min} \text { during exercise } \\
\left(3 \mathrm{~mL} \cdot \mathrm{kg}^{-1}\right)\end{array}$ & $0.42[-0.33,1.17]$ \\
\hline Glace et al., 2019 i [78] & CS & $10 / \mathrm{M}$ & 35.0 & $\#$ & $60.3 *$ & Lab & $\begin{array}{l}120 \text { min of cycling at VT with } \\
\text { interspersed higher-intensity } \\
\text { intervals + a } 3 \mathrm{~km} \text { TT }\end{array}$ & $\begin{array}{l}5.9 \% \text { CHO-E (Gatorade, PepsiCo, } \\
\text { Purchase, NY, USA) at a rate of } \\
1 \% \text { of BM each h (male subjects) }\end{array}$ & $0.28[-0.60,1.16]$ \\
\hline Glace et al., 2019 ii [78] & CS & $10 / \mathrm{F}$ & 42.0 & $\#$ & $46.5^{*}$ & $\mathrm{Lab}$ & $\begin{array}{l}120 \text { min of cycling at VT with } \\
\text { interspersed higher-intensity } \\
\text { intervals + a } 3 \mathrm{~km} \mathrm{TT}\end{array}$ & $\begin{array}{c}5.9 \% \text { CHO-E (Gatorade, PepsiCo, } \\
\text { Purchase, NY, USA) at a rate of } \\
1 \% \text { of BM each } h \text { (female } \\
\text { subjects) }\end{array}$ & $0.38[-0.51,1.26]$ \\
\hline
\end{tabular}


Table 1. Cont.

\begin{tabular}{|c|c|c|c|c|c|c|c|c|c|}
\hline Study/Trial † & Study Design & $\begin{array}{c}N / \\
\text { Gender }\end{array}$ & $\begin{array}{l}\text { Age } \\
\text { (year) }\end{array}$ & $\begin{array}{c}\text { BMI } \\
\left(\mathbf{k g} \cdot \mathbf{m}^{-2}\right)\end{array}$ & $\begin{array}{c}\dot{\mathrm{V} \mathrm{O}_{2} \mathrm{max}} \\
\left(\mathrm{mL} \cdot \mathrm{kg}^{-1} \cdot \mathrm{min}^{-1}\right)\end{array}$ & $\begin{array}{l}\text { Lab/ } \\
\text { Field }\end{array}$ & $\begin{array}{c}\text { Exercise Task } \\
\text { (Brief Description) }\end{array}$ & $\begin{array}{l}\text { CHO Supplementation } \\
\text { (Brief Description) }\end{array}$ & $\begin{array}{l}\text { SMD IV, Random } \\
{[95 \% \text { CI })}\end{array}$ \\
\hline Glace et al., 2019 iii [78] & CS & $10 / \mathrm{M}$ & 35.0 & $\#$ & $60.3 *$ & Lab & $\begin{array}{c}120 \text { min of cycling at VT with } \\
\text { interspersed higher-intensity } \\
\text { intervals + a } 3 \mathrm{~km} \mathrm{TT}+\text { a ride } \\
\text { to exhaustion at RCT }\end{array}$ & $\begin{array}{l}5.9 \% \text { CHO-E (Gatorade, PepsiCo, } \\
\text { Purchase, NY, USA) at a rate of } \\
1 \% \text { of BM each h (male subjects) }\end{array}$ & $0.38[-0.51,1.26]$ \\
\hline Glace et al., 2019 iv [78] & CS & $10 / \mathrm{F}$ & 42.0 & $\#$ & $46.5^{*}$ & $\mathrm{Lab}$ & $\begin{array}{l}120 \text { min of cycling at VT with } \\
\text { interspersed higher-intensity } \\
\text { intervals + a } 3 \mathrm{~km} \text { TT + a ride } \\
\text { to exhaustion at RCT }\end{array}$ & $\begin{array}{c}5.9 \% \text { CHO-E (Gatorade, PepsiCo, } \\
\text { Purchase, NY, USA) at a rate of } \\
1 \% \text { of BM each h (female } \\
\text { subjects) }\end{array}$ & $-0.02[-0.89,0.86]$ \\
\hline Goedecke et al., 2013 [79] & CS & $22 / \mathrm{M}$ & 24.0 & 25.0 & 51.8 & $\mathrm{Lab}$ & $\begin{array}{l}\text { ( } 2 \times \text { Illinois agility run) }+ \text { SSM } \\
\text { (LIST } \sim 90 \text { min) + Illinois agility } \\
\text { run + timed run to fatigue } \\
(20 \mathrm{~m} \text { runs at progressively } \\
\text { increasing speeds at the point } \\
\text { where two consecutive shuttles } \\
\text { could not be completed) }\end{array}$ & $\begin{array}{l}\text { 7\% CHO-E drink (SUC: } \\
\text { Energade, Tiger Consumer Br\&s } \\
\text { Ltd., Bryanston, Johannesburg, } \\
\text { South Africa), } 250 \mathrm{~mL} \text { prior to } \\
\text { the warm-up and following the } \\
\text { 3rd } 15 \text {-min exercise bout and } \\
50 \mathrm{~mL} \text { during the } 90 \text { sec-break } \\
\text { separating each } 15-\text { min exercise } \\
\text { bout (total: } 700 \mathrm{~mL}, \mathrm{CHO} 49 \mathrm{~g} \text { ) }\end{array}$ & $0.39[-0.21,0.99]$ \\
\hline Gui et al., 2017 [80] & CS & $11 / \mathrm{F}$ & 32.4 & 21.0 & 49.0 & $\mathrm{Lab}$ & $\begin{array}{l}5 \mathrm{~km} \text { at } 70 \% \dot{\mathrm{VO}}_{2} \max +16 \mathrm{~km} \\
\text { performance run, TA: } 22.0^{\circ} \mathrm{C}\end{array}$ & $\begin{array}{c}\text { 6\% CHO-E (SUC: Coca-Cola, } \\
\text { HK), } 150 \text { mL at } 0 \mathrm{~km} \text { and every } \\
2.5 \mathrm{~km}(\mathrm{CHO} \text { ingestion rate } \\
\left.\sim 36 \mathrm{~g} \cdot \mathrm{h}^{-1}\right)\end{array}$ & $0.47[-0.38,1.32]$ \\
\hline Heesch et al., 2014 i [81] & CS & $8 / \mathrm{M}$ & 34.5 & 24.8 & 56.8 & $\mathrm{Lab}$ & $\begin{array}{l}\text { cycling at } 60 \% \text { of the Wmax for } \\
120 \text { min }+ \text { a } 10 \mathrm{~km} \text { cycling } \\
\text { performance trial, TA: } 21.5{ }^{\circ} \mathrm{C}\end{array}$ & $\begin{array}{l}3 \% \text { CHO solution (MD), } 250 \mathrm{~mL} \\
\text { every } 15 \text { min during the } 2-\mathrm{hr} \\
\text { cycling bout }\end{array}$ & $0.28[-0.71,1.26]$ \\
\hline Heesch et al., 2014 ii [81] & CS & $8 / \mathrm{M}$ & 34.5 & 24.8 & 56.8 & $\mathrm{Lab}$ & $\begin{array}{l}\text { cycling at } 60 \% \text { of the Wmax for } \\
120 \mathrm{~min}+\mathrm{a} 10 \mathrm{~km} \text { cycling } \\
\text { performance trial, TA: } 21.5^{\circ} \mathrm{C}\end{array}$ & $\begin{array}{l}6 \% \text { CHO solution (MD), } 250 \mathrm{~mL} \\
\text { every } 15 \text { min during the } 1 \mathrm{st} \mathrm{h}, \\
\text { followed by } 250 \mathrm{~mL} \text { of an } \\
\text { artificially sweetened placebo } \\
\text { beverage every } 15 \mathrm{~min} \text { during } \\
\text { the } 2 \text { nd h of cycling }\end{array}$ & $0.17[-0.81,1.15]$ \\
\hline Heesch et al., 2014 iii [81] & CS & $8 / \mathrm{M}$ & 34.5 & 24.8 & 56.8 & Lab & $\begin{array}{l}\text { cycling at } 60 \% \text { of the Wmax for } \\
120 \mathrm{~min}+\mathrm{a} 10 \mathrm{~km} \text { cycling } \\
\text { performance trial, TA: } 21.5^{\circ} \mathrm{C}\end{array}$ & $\begin{array}{l}\text { artificially sweetened placebo } \\
\text { beverage of a } 250 \mathrm{~mL} \text { every } \\
15 \mathrm{~min} \text { during the } 1 \mathrm{st} \text {, followed } \\
\text { by } 250 \mathrm{~mL} \text { of an } 6 \% \mathrm{CHO} \\
\text { solution (MD) every } 15 \mathrm{~min} \\
\text { during the } 2 \text { nd h of cycling }\end{array}$ & $0.31[-0.68,1.30]$ \\
\hline
\end{tabular}


Table 1. Cont.

\begin{tabular}{|c|c|c|c|c|c|c|c|c|c|}
\hline Study/Trial † & Study Design & $\begin{array}{l}N / \\
\text { Gender }\end{array}$ & $\begin{array}{l}\text { Age } \\
\text { (year) }\end{array}$ & $\begin{array}{c}\text { BMI } \\
\left(\mathbf{k g} \cdot \mathbf{m}^{-2}\right)\end{array}$ & $\begin{array}{c}\dot{\mathrm{V} \mathrm{O}_{2} \mathrm{max}} \\
\left(\mathrm{mL} \cdot \mathrm{kg}^{-1} \cdot \mathrm{min}^{-1}\right)\end{array}$ & $\begin{array}{l}\text { Lab/ } \\
\text { Field }\end{array}$ & $\begin{array}{c}\text { Exercise Task } \\
\text { (Brief Description) }\end{array}$ & $\begin{array}{l}\text { CHO Supplementation } \\
\text { (Brief Description) }\end{array}$ & $\begin{array}{l}\text { SMD IV, Random } \\
{[95 \% \mathrm{CI})}\end{array}$ \\
\hline $\begin{array}{l}\text { Hulston \& Jeukendrup } \\
2008[82]\end{array}$ & CS & $10 / \mathrm{M}$ & 27.0 & $\#$ & 65.7 & $\mathrm{Lab}$ & $\begin{array}{l}105 \text { min of SS cycling at } 62 \% \\
\mathrm{~V}_{2} \text { max followed by a TT to } \\
\text { complete a set amount of work } \\
(688 \pm 56 \mathrm{~kJ}) \text { as fast as possible }\end{array}$ & $\begin{array}{l}6.4 \% \mathrm{CHO} \text { solution }(\mathrm{GL}) \\
5.5 \mathrm{~mL} \cdot \mathrm{kg}^{-1} \text { at the onset of } \\
\text { exercise and } 2 \mathrm{~mL} \cdot \mathrm{kg}^{-1} \text { at } \\
\text { subsequent } 15-\mathrm{min} \text { intervals until } \\
\text { completion of the SS exercise }\end{array}$ & $0.50[-0.40,1.39]$ \\
\hline $\begin{array}{l}\text { Hulston \& Jeukendrup } \\
2009[83]\end{array}$ & CS & $10 / \mathrm{M}$ & 28.0 & $\#$ & 61.7 & $\mathrm{Lab}$ & $\begin{array}{l}120 \text { min of SS cycling at } 61 \% \\
\text { VO }_{2} \text { max followed by a TT to } \\
\text { complete a set amount of work } \\
(847 \pm 78 \mathrm{~kJ}) \text { as fast as possible }\end{array}$ & $\begin{array}{l}6 \% \text { CHO-E solution (GL:FRU in a } \\
\text { ratio of 2:1), } 600 \mathrm{~mL} \text { at the onset } \\
\text { of exercise and } 150 \mathrm{~mL} \text { every } \\
15 \mathrm{~min} \text { thereafter }\end{array}$ & $1.35[0.36,2.35]$ \\
\hline Ivy et al., 1983 [84] & CS & $10 / \mathrm{M}$ & 23.8 & 23.3 & 60.4 & $\mathrm{Lab}$ & TE walking at $45 \% \dot{\mathrm{VO}}_{2} \max$ & $\begin{array}{c}\text { 20\% GL-polymer solution } \\
\text { (Polycose, Ross Laboratories, } \\
\text { Columbus, OH), }\left(2 \mathrm{kcal} \cdot \mathrm{mL}^{-1}\right), \\
\text { in four equally divided dosages } \\
60,90,120 \text {, and } 150 \mathrm{~min} \\
\text { following the start of exercise, } \\
\text { (total: GL-polymer } 120 \mathrm{~g} \text { ), served } \\
\text { at } 4.0^{\circ} \mathrm{C}\end{array}$ & $0.86[-0.07,1.78]$ \\
\hline Jarvis et al., 1999 [85] & CS & $10 / \mathrm{F}$ & 30.4 & 22.4 & 47.1 & $\mathrm{Lab}$ & $\begin{array}{c}50 \text { min of cycle ergometry at } \\
80 \% \dot{\mathrm{VO}}_{2} \max +\text { WAT }\end{array}$ & $\begin{array}{l}7 \% \text { CHO solution (GL-polymer } \\
\text { containing MD: Exceed), } \\
2 \mathrm{~mL} \cdot \mathrm{kg}^{-1} \mathrm{BM} \text { at } 10,20,30 \text {, and } \\
40-\mathrm{min} \text { intervals throughout the } \\
50 \text { min of exercise }\end{array}$ & $0.08[-0.79,0.96]$ \\
\hline Jeukendrup et al., 1997 [86] & CS & $\begin{array}{l}19 / 17 \mathrm{M} \\
2 \mathrm{~F}\end{array}$ & $\begin{array}{l}23.0 \mathrm{M} \\
\text { and } \\
21.0 \mathrm{~F}\end{array}$ & $\begin{array}{l}31.3 \mathrm{M} \text { and } \\
18.8 \mathrm{~F}\end{array}$ & $\begin{array}{l}72.9 \mathrm{M} \text { and } \\
64.2 \mathrm{~F}\end{array}$ & Lab & $\begin{array}{c}\text { amount of cycling work } \\
(0.75 \times \text { Wmax } \times 3600) \text { as fast } \\
\text { as possible }(\sim 60 \mathrm{~min}), \\
\text { TA: } 20.0^{\circ} \mathrm{C}\end{array}$ & $\begin{array}{c}\text { 7.6\% CHO-E solution (Isostar, } \\
\text { Sandoz Nutrition, Switzerl\&), } \\
8 \mathrm{~mL} \cdot \mathrm{kg}^{-1} \text { at the } 5-\mathrm{min} \text { warm-up } \\
\text { period and } 2 \mathrm{~mL} \cdot \mathrm{kg}^{-1} \text { upon } \\
\text { achievement of } 25 \%, 50 \% \text { and } \\
75 \% \text { of the work set }\end{array}$ & $0.54[-0.11,1.19]$ \\
\hline Kang et al., 1995 [87] & CS & $7 / \mathrm{M}$ & 23.0 & 23.3 & $61.6 *$ & $\mathrm{Lab}$ & $\mathrm{TE}$ cycling at $71 \pm 1 \% \dot{\mathrm{VO}}_{2} \max$ & $\begin{array}{l}\text { 6\% GL-SUC solution (Gatorade, } \\
\text { Quaker Oats Co.) every } 20 \mathrm{~min} \\
\text { at a rate of } 0.6 \mathrm{~g} \cdot \mathrm{kg}^{-1} \cdot \mathrm{h}^{-1}\end{array}$ & $0.61[-0.47,1.69]$ \\
\hline Kang et al., 1996 [88] & CS & $7 / \mathrm{M}$ & 23.0 & 23.3 & $61.2 *$ & Lab & TE cycling at $70 \% \dot{\mathrm{VO}}_{2} \max$ & $\begin{array}{l}6 \% \text { GL-SUC solution (Gatorade, } \\
\text { Quaker Oats Co.) every } 20 \mathrm{~min} \\
\text { at a rate of } 0.6 \mathrm{~g} \cdot \mathrm{kg}^{-1} \cdot \mathrm{h}^{-1}\end{array}$ & $0.48[-0.59,1.55]$ \\
\hline Khanna \& Manna 2005 [89] & CS & $10 / \mathrm{M}$ & 25.8 & 21.0 & 54.4 & Lab & $\begin{array}{c}\text { TE running at } 70 \% \dot{\mathrm{VO}}_{2} \max , \\
\text { TA: } 25.0^{\circ} \mathrm{C}\end{array}$ & $\begin{array}{l}5 \% \text { CHO-E solution, } 100 \mathrm{~mL} \text { at } \\
\text { 15-min intervals until completion } \\
\text { of the exercise }\end{array}$ & $2.10[0.96,3.24]$ \\
\hline
\end{tabular}


Table 1. Cont.

\begin{tabular}{|c|c|c|c|c|c|c|c|c|c|}
\hline Study/Trial $†$ & Study Design & $\begin{array}{c}N / \\
\text { Gender }\end{array}$ & $\begin{array}{c}\text { Age } \\
\text { (year) }\end{array}$ & $\begin{array}{c}\text { BMI } \\
\left(\mathbf{k g} \cdot \mathrm{m}^{-2}\right)\end{array}$ & $\begin{array}{c}\dot{\mathrm{VO}_{2} \max } \\
\left(\mathrm{mL} \cdot \mathrm{kg}^{-1} \cdot \mathrm{min}^{-1}\right)\end{array}$ & $\begin{array}{l}\text { Lab/ } \\
\text { Field }\end{array}$ & $\begin{array}{c}\text { Exercise Task } \\
\text { (Brief Description) }\end{array}$ & $\begin{array}{c}\text { CHO Supplementation } \\
\text { (Brief Description) }\end{array}$ & $\begin{array}{l}\text { SMD IV, Random } \\
{[95 \% \text { CI }]}\end{array}$ \\
\hline King et al., 2018 i [90] & CS & $10 / \mathrm{M}$ & 30.7 & $\#$ & 61.6 & $\mathrm{Lab}$ & $\begin{array}{c}120 \text { min of cycling at } 77 \% \\
\dot{\mathrm{VO}}_{2} \mathrm{max}+\mathrm{a} 30 \text {-min self-paced } \\
\text { TT }\end{array}$ & $\begin{array}{l}\text { CHO-E, CHO ingestion rate } 60 \mathrm{~g} \cdot \mathrm{h}^{-1} \\
\text { (D-GL; Thornton \& Ross Ltd., } \\
\text { Huddersfield, UK), } 250 \mathrm{~mL} \text { solution } \\
\text { every } 15 \mathrm{~min} \text {, starting at } 15 \text {-min into } \\
\text { the exercise protocol }\end{array}$ & $0.43[-0.46,1.32]$ \\
\hline King et al., 2018 ii [90] & CS & $10 / \mathrm{M}$ & 30.7 & $\#$ & 61.6 & $\mathrm{Lab}$ & $\begin{array}{c}120 \text { min of cycling at } 77 \% \\
\dot{\mathrm{VO}}_{2} \mathrm{max}+\text { a } 30 \text {-min self-paced } \\
\text { TT }\end{array}$ & $\begin{array}{l}\text { CHO-E, CHO ingestion rate } 75 \mathrm{~g} \cdot \mathrm{h}^{-1}, \\
\text { (D-GL; Thornton \& Ross Ltd., } \\
\text { Huddersfield, UK), } 250 \mathrm{~mL} \text { solution } \\
\text { every } 15 \mathrm{~min} \text {, starting at } 15 \mathrm{~min} \text { into } \\
\text { the exercise protocol }\end{array}$ & $0.19[-0.69,1.07]$ \\
\hline King et al., 2018 iii [90] & CS & $10 / \mathrm{M}$ & 30.7 & $\#$ & 61.6 & $\mathrm{Lab}$ & $\begin{array}{c}120 \text { min of cycling at } 77 \% \\
\dot{\mathrm{VO}}_{2} \mathrm{max}+\mathrm{a} 30 \text {-min self-paced } \\
\text { TT }\end{array}$ & $\begin{array}{c}\text { CHO-E, CHO ingestion rate } 90 \mathrm{~g} \cdot \mathrm{h}^{-1}, \\
\text { GL:FRU in a ratio of 2:1 (GL:D-GL; } \\
\text { Thornton \& Ross Ltd., Huddersfield, } \\
\text { UK \& FRU: Danisco, Kettering, UK), } \\
250 \mathrm{~mL} \text { solution every } 15 \mathrm{~min}, \\
\text { starting at } 15 \text { min into the exercise } \\
\text { protocol }\end{array}$ & $0.83[-0.10,1.75]$ \\
\hline King et al., 2018 iv [90] & CS & $10 / \mathrm{M}$ & 30.7 & $\#$ & 61.6 & $\mathrm{Lab}$ & $\begin{array}{c}120 \text { min of cycling at } 77 \% \\
\dot{\mathrm{VO}}_{2} \mathrm{max}+\text { a } 30 \text {-min self-paced } \\
\text { TT }\end{array}$ & $\begin{array}{l}\text { CHO-E, GL:FRU in a ratio of 2:1, } \\
\text { CHO ingestion rate } 112.5 \mathrm{~g} \cdot \mathrm{h}^{-1} \\
\text { (GL:D-GL; Thornton \& Ross Ltd., } \\
\text { Huddersfield, UK \& FRU: Danisco, } \\
\text { Kettering, UK), } 250 \mathrm{~mL} \text { solution } \\
\text { every } 15 \text { min, starting at } 15 \text { min into } \\
\text { the exercise protocol }\end{array}$ & $0.58[-0.32,1.48]$ \\
\hline King et al., 2019 i [91] & CS & $11 / \mathrm{M}$ & 30.3 & 24.2 & 60.0 & $\mathrm{Lab}$ & $\begin{array}{c}180 \text { min of cycling at } 60 \% \\
\dot{\mathrm{VO}}_{2} \mathrm{max}+\mathrm{a} 30 \text {-min self-paced } \\
\text { TT }\end{array}$ & $\begin{array}{l}\text { CHO-E, GL:FRU in a ratio of 2:1, } \\
\text { CHO ingestion rate } 80 \mathrm{~g} \cdot \mathrm{h}^{-1} \\
\text { (GL:D-GL; Thornton \& Ross Ltd., } \\
\text { Huddersfield, UK \& FRU: Danisco, } \\
\text { Kettering, UK), } 250 \mathrm{~mL} \text { solution } \\
\text { every } 15 \mathrm{~min} \text { starting at } 15 \text { min into } \\
\text { the exercise protocol }\end{array}$ & $0.92[0.03,1.81]$ \\
\hline King et al., 2019 ii [91] & CS & $11 / \mathrm{M}$ & 30.3 & 24.2 & 60.0 & $\mathrm{Lab}$ & $\begin{array}{c}180 \text { min of cycling at } 60 \% \\
\dot{\mathrm{VO}}_{2} \text { max }+ \text { a } 30-\text {-min self-paced } \\
\text { TT }\end{array}$ & $\begin{array}{c}\text { CHO-E, GL:FRU in a ratio of 2:1, } \\
\text { CHO ingestion rate } 90 \mathrm{~g} \cdot \mathrm{h}^{-1} \\
\text { (GL:D-GL; Thornton \& Ross Ltd., } \\
\text { Huddersfield, UK \& FRU: Danisco, } \\
\text { Kettering, UK), } 250 \mathrm{~mL} \text { solution } \\
\text { every } 15 \text { min, starting at } 15 \text { min into } \\
\text { the exercise protocol }\end{array}$ & $1.09[0.18,2.00]$ \\
\hline
\end{tabular}


Table 1. Cont.

\begin{tabular}{|c|c|c|c|c|c|c|c|c|c|}
\hline Study/Trial † & Study Design & $\begin{array}{c}N / \\
\text { Gender }\end{array}$ & $\begin{array}{c}\text { Age } \\
\text { (year) }\end{array}$ & $\begin{array}{c}\text { BMI } \\
\left(\mathbf{k g} \cdot \mathbf{m}^{-2}\right)\end{array}$ & $\begin{array}{c}\dot{\mathrm{V} \mathrm{O}_{2} \max } \\
\left(\mathrm{mL} \cdot \mathrm{kg}^{-1} \cdot \mathrm{min}^{-1}\right)\end{array}$ & $\begin{array}{l}\text { Lab/ } \\
\text { Field }\end{array}$ & $\begin{array}{c}\text { Exercise Task } \\
\text { (Brief Description) }\end{array}$ & $\begin{array}{l}\text { CHO Supplementation } \\
\text { (Brief Description) }\end{array}$ & $\begin{array}{l}\text { SMD IV, Random } \\
{[95 \% \mathrm{CI}]}\end{array}$ \\
\hline King et al., 2019 iii [91] & CS & $11 / \mathrm{M}$ & 30.3 & 24.2 & 60.0 & $\mathrm{Lab}$ & $\begin{array}{c}180 \text { min of cycling at } 60 \% \\
\mathrm{VO}_{2} \max +\text { a } 30-\text { min self-paced } \\
\text { TT }\end{array}$ & $\begin{array}{l}\text { CHO-E, GL:FRU in a ratio of 2:1, } \\
\text { CHO ingestion rate } 100 \mathrm{~g} \cdot \mathrm{h}^{-1} \\
\text { (GL:D-GL; Thornton \& Ross Ltd., } \\
\text { Huddersfield, UK \& FRU: Danisco, } \\
\text { Kettering, UK), } 250 \mathrm{~mL} \text { solution } \\
\text { every } 15 \mathrm{~min} \text {, starting at } 15 \text { min into } \\
\text { the exercise protocol }\end{array}$ & $0.58[-0.27,1.44]$ \\
\hline Kingwell et al., 1989 [92] & CS & $9 / \mathrm{M}$ & 23.0 & $\#$ & 74.1 & $\mathrm{Lab}$ & $\begin{array}{c}160 \text { min of cycling at } 65 \% \\
\dot{\mathrm{VO}}_{2} \mathrm{max}+5 \text { min rest }+\mathrm{TE} \text { at } \\
110 \% \dot{\mathrm{VO}}_{2} \max \\
\text { TA: } 20.0-22.0^{\circ} \mathrm{C}\end{array}$ & $\begin{array}{c}10 \% \text { GL-polymer solution (Polycose), } \\
200 \mathrm{~mL} \text { at the start of exercise and at } \\
20 \text {-min intervals thereafter }\end{array}$ & $0.53[-0.42,1.47]$ \\
\hline Learsi et al., 2019 i [93] & CS & $9 / \mathrm{M}$ & 28.0 & 24.9 & 41.2 & $\mathrm{Lab}$ & $\begin{array}{l}105 \mathrm{~min} \text { of constant-load } \\
\text { cycling }(50 \% \text { of the difference } \\
\text { between the } 1 \text { st and } 2 \text { nd } \\
\text { LT) + a } 10 \mathrm{~km} \text { TT, TA: } 21.0^{\circ} \mathrm{C}\end{array}$ & $\begin{array}{l}8 \% \text { CHO beverage (MD, } 2 \mathrm{~mL} \cdot \mathrm{kg}^{-1} \\
\text { of BM: Neonutri, Poços de Caldas, } \\
\text { Brazil) immediately prior to exercise, } \\
\text { every } 15 \text { min throughout the } \\
\text { constant-load exercise and at the } 5 \text { th } \\
\mathrm{km} \text { point during the } 10-\mathrm{km} \mathrm{TT}\end{array}$ & $0.89[-0.09,1.87]$ \\
\hline Learsi et al., 2019 ii [93] & CS & $9 / \mathrm{M}$ & 28.0 & 24.9 & 41.2 & $\mathrm{Lab}$ & $\begin{array}{l}105 \mathrm{~min} \text { of constant-load } \\
\text { cycling }(50 \% \text { of the difference } \\
\text { between the } 1 \text { st and } 2 \text { nd } \\
\text { LT) + a } 10 \mathrm{~km} \mathrm{TT}, \mathrm{TA}: 21.0^{\circ} \mathrm{C}\end{array}$ & $\begin{array}{l}\text { 8\% CHO beverage (MD, } 2 \mathrm{~mL} \cdot \mathrm{kg}^{-1} \\
\text { of BM: Neonutri, Poços de Caldas, } \\
\text { Brazil) immediately prior to exercise, } \\
\text { every } 15 \text { min throughout the } \\
\text { constant-load exercise and at the } 5 \text { th } \\
\text { km point during the } 10-\mathrm{km} \text { TT } \\
\text { (overnight fast trial) }\end{array}$ & $2.31[1.05,3.57]$ \\
\hline Lugo et al., 1993 [94] & CS & $9 / \mathrm{M}$ & 23.0 & $\#$ & $63.7^{*}$ & $\mathrm{Lab}$ & $\begin{array}{c}120 \text { min of cycling at } 70 \% \\
\dot{\mathrm{VO}}_{2} \text { peak }+5 \text { min rest }+\mathrm{TT} \\
\text { distance (calculated as the } \\
\text { distance traveled if } 80 \% \\
\mathrm{VO}_{2} \text { peak was maintained for } \\
30 \text { min), TA: } 22.0^{\circ} \mathrm{C}\end{array}$ & $\begin{array}{c}\text { 7\% CHO-E solution (Fluid } \\
\text { Replacement Energy Drinks, Ross } \\
\text { Laboratories, Columbus, OH), } \\
0.4 \mathrm{~g} \cdot \mathrm{kg}^{-1} \text { at } 0 \text { min and every } 30 \mathrm{~min} \\
\text { until the } 120 \text { th min }\end{array}$ & $0.88[-0.10,1.86]$ \\
\hline $\begin{array}{c}\text { Martínez-Lagunas et al., } \\
2010 \text { [95] }\end{array}$ & CS & $\begin{array}{l}12 / 7 \mathrm{M} \\
5 \mathrm{~F}\end{array}$ & 28.3 & 22.1 & $\begin{array}{l}63.2 \mathrm{M} \text { and } \\
49.2 \mathrm{~F}\end{array}$ & $\mathrm{Lab}$ & $\begin{array}{c}\text { cycling at varied intensities, } \\
55-75 \% \dot{\mathrm{V}}_{2} \text { max for } 150 \mathrm{~min}+ \\
\text { at } 80 \% \dot{\mathrm{VO}}_{2} \text { max until fatigued, } \\
\text { TA: } 21.0-23.0^{\circ} \mathrm{C}\end{array}$ & $\begin{array}{l}\text { 6\% CHO-E solution, } 0.7 \mathrm{~g} \cdot \mathrm{kg}^{-1} \cdot \mathrm{h}^{-1} \\
\quad \text { every } 20 \mathrm{~min}(255.4 \pm 9.1 \mathrm{~mL})\end{array}$ & $0.69[-0.14,1.52]$ \\
\hline Maughan et al., 1989 [96] & CS & $6 / \mathrm{M}$ & 29.0 & 22.3 & 53.0 & $\mathrm{Lab}$ & TE cycling at $70 \% \dot{\mathrm{V}}_{2} \max$ & $\begin{array}{l}4 \% \text { CHO solution (GL-E: Dioralyte, } \\
\text { Rorer Health Care Ltd., Eastbourne, } \\
\text { UK), } 100 \mathrm{~mL} \text { at } 0 \text {-min and at } 10 \text {-min } \\
\text { intervals }\end{array}$ & $0.51[-0.65,1.66]$ \\
\hline
\end{tabular}


Table 1. Cont.

\begin{tabular}{|c|c|c|c|c|c|c|c|c|c|}
\hline Study/Trial † & Study Design & $\begin{array}{c}N / \\
\text { Gender }\end{array}$ & $\begin{array}{l}\text { Age } \\
\text { (year) }\end{array}$ & $\begin{array}{c}\text { BMI } \\
\left(\mathbf{k g} \cdot \mathbf{m}^{-2}\right)\end{array}$ & $\begin{array}{c}\dot{\mathrm{VO}} \mathrm{O}_{2} \max \\
\left(\mathrm{mL} \cdot \mathrm{kg}^{-1} \cdot \mathrm{min}^{-1}\right)\end{array}$ & $\begin{array}{l}\text { Lab/ } \\
\text { Field }\end{array}$ & $\begin{array}{c}\text { Exercise Task } \\
\text { (Brief Description) }\end{array}$ & $\begin{array}{l}\text { CHO Supplementation } \\
\text { (Brief Description) }\end{array}$ & $\begin{array}{l}\text { SMD IV, Random } \\
{[95 \% \text { CI })}\end{array}$ \\
\hline McConell et al., 1996 [97] & CS & $8 / \mathrm{M}$ & 23.0 & $\#$ & $69.2 *$ & $\mathrm{Lab}$ & $\begin{array}{c}120 \text { min of cycling at } 70 \pm 1 \% \\
\mathrm{VO}_{2} \text { peak, }+15 \text { min all out } \\
\text { performance, TA: } 20.0-22.0^{\circ} \mathrm{C}\end{array}$ & $\begin{array}{l}7 \% \text { CHO-E drink (Sport Plus, } \\
\text { Cadbury Schweppes Pty. Ltd., } \\
\text { Melbourne, Australia), } 250 \mathrm{~mL} \text { at } \\
0 \text { min and at } 15-\text { min intervals } \\
\text { until the } 120 \text { th min }\end{array}$ & $1.02[-0.04,2.08]$ \\
\hline McConell et al., 1999 [98] & CS & $8 / \mathrm{M}$ & 22.0 & $\#$ & $66.9^{*}$ & $\mathrm{Lab}$ & $\begin{array}{c}\mathrm{TE} \text { cycling at } 69 \pm 1 \% \\
\dot{\mathrm{VO}}_{2} \text { peak }\end{array}$ & $\begin{array}{l}8 \% \mathrm{CHO} \text { solution, } 250 \mathrm{~mL} \\
\text { immediately prior to exercise } \\
\text { and at } 15-\mathrm{min} \text { intervals thereafter }\end{array}$ & $0.97[-0.08,2.03]$ \\
\hline McConell et al., 2000 [99] & CS & $13 / \mathrm{M}$ & 24.0 & 23.0 & 65.7 & $\mathrm{Lab}$ & $\begin{array}{c}\text { TE cycling at } 83 \pm 1 \% \\
\dot{\mathrm{VO}}_{2} \text { peak, TA: } 19.0-22.0^{\circ} \mathrm{C}\end{array}$ & $\begin{array}{c}6 \% \mathrm{CHO} \text { (D-GL) solution, } \\
7 \mathrm{~mL} \cdot \mathrm{kg}^{-1} \text { immediately prior to } \\
\text { exercise and } 3.5 \mathrm{~mL} \cdot \mathrm{kg}^{-1} \text { at } \\
15 \text {-min intervals thereafter }\end{array}$ & $-0.08[-0.85,0.69]$ \\
\hline $\begin{array}{l}\text { McGawley et al., } \\
2012 \text { [100] }\end{array}$ & CS & $\begin{array}{l}10 / 6 \mathrm{M} \\
\quad 4 \mathrm{~F}\end{array}$ & $\begin{array}{l}25.0 \mathrm{M} \\
\text { and } \\
26.0 \mathrm{~F}\end{array}$ & $\#$ & $\begin{array}{l}62.9 * \mathrm{M} \text { and } \\
61.9^{*} \mathrm{~F}\end{array}$ & $\mathrm{Lab}$ & $\begin{array}{l}\text { simulated Olympic-distance } \\
\text { triathlon with 3-min transition } \\
\text { period between sections } \\
(1500 \mathrm{~m} \text { swimming }+40 \mathrm{~km} \\
\text { cycling at } 75 \% \text { of MAP were of } \\
\text { fixed intensity while the } 10 \mathrm{~km} \\
\text { run section was completed as a } \\
\text { TT), TA: } 15.9^{\circ} \mathrm{C}\end{array}$ & $\begin{array}{l}\text { 14.4\% CHO-E solution (MD:FRU } \\
\text { in a ratio of 2:1: EnergySource } \\
\text { Fresh Citrus flavor, H5 Ltd., } \\
\text { Leicestershire, UK), } 202 \pm 20 \mathrm{~mL} \\
2 \text { min prior to completing every } \\
\text { quarter of the cycle section }\end{array}$ & $0.40[-0.48,1.29]$ \\
\hline $\begin{array}{l}\text { Millard-Stafford et al., } \\
1990 \text { [101] }\end{array}$ & CS & $10 / \mathrm{M}$ & 29.6 & 24.3 & 67.0 & $\mathrm{Lab}$ & $\begin{array}{c}\text { simulated triathlon: } 1.5 \mathrm{~km} \\
\text { swimming }+40 \mathrm{~km} \\
\text { cycling }+10 \mathrm{~km} \text { running, } \\
\text { TA: } 30.0^{\circ} \mathrm{C}\end{array}$ & $\begin{array}{l}\text { 7\% CHO-E solution ( } 5 \% \\
\text { GL-polymer }+2 \% \text { FRU: Exceed, } \\
\text { Ross Laboratories, Columbus, } \\
\text { OH), } 2 \mathrm{~mL} \cdot \mathrm{kg}^{-1} \text { following the } \\
\text { swim, at } 8-\mathrm{km}_{\text {intervals during }} \\
\text { cycling, and at } 3.2-\mathrm{km} \text { intervals } \\
\text { during running }\end{array}$ & $0.14[-0.74,1.02]$ \\
\hline $\begin{array}{l}\text { Millard-Stafford et al., } \\
2005 \text { i [102] }\end{array}$ & CS & $10 / \mathrm{M}$ & 23.7 & 21.2 & 76.9 & Field & $\begin{array}{l}\text { the final } 5 \mathrm{~km} \text { of a } 32-\mathrm{km} \text { run at } \\
\qquad 25.6^{\circ} \mathrm{C}\end{array}$ & $\begin{array}{l}\text { 6\% CHO-E beverage (SUC:GL: } \\
\text { Gatorade, The Quaker Oats Co., } \\
\text { Chicago, IL, USA), } 400 \mathrm{~mL} \\
15 \text { min prior to exercise and } \\
250 \mathrm{~mL} \text { every } 5 \mathrm{~km} \text { thereafter }\end{array}$ & $0.50[-0.39,1.40]$ \\
\hline $\begin{array}{l}\text { Millard-Stafford et al., } \\
2005 \text { ii [102] }\end{array}$ & CS & $10 / \mathrm{M}$ & 23.7 & 21.2 & 76.9 & Field & $\begin{array}{l}\text { the final } 5 \mathrm{~km} \text { of a } 32-\mathrm{km} \text { run at } \\
\qquad 25.6^{\circ} \mathrm{C}\end{array}$ & $\begin{array}{c}8 \% \text { CHO-E beverage } \\
\text { (3.5\% FRU + 2.5\%GL + 2\% MD: } \\
\text { PowerAde, The Coca-Cola Co., } \\
\text { Atlanta, GA), } 400 \mathrm{~mL} 15 \mathrm{~min} \\
\text { prior to exercise and } 250 \mathrm{~mL} \\
\text { every } 5 \mathrm{~km} \text { thereafter }\end{array}$ & $0.62[-0.29,1.52]$ \\
\hline
\end{tabular}


Table 1. Cont.

\begin{tabular}{|c|c|c|c|c|c|c|c|c|c|}
\hline Study/Trial † & Study Design & $\begin{array}{c}N / \\
\text { Gender }\end{array}$ & $\begin{array}{c}\text { Age } \\
\text { (year) }\end{array}$ & $\begin{array}{c}\text { BMI } \\
\left(\mathbf{k g} \cdot \mathrm{m}^{-2}\right)\end{array}$ & $\begin{array}{c}\dot{\mathrm{VO}_{2}} \max \\
\left(\mathrm{mL} \cdot \mathrm{kg}^{-1} \cdot \mathrm{min}^{-1}\right)\end{array}$ & $\begin{array}{l}\text { Lab/ } \\
\text { Field }\end{array}$ & $\begin{array}{c}\text { Exercise Task } \\
\text { (Brief Description) }\end{array}$ & $\begin{array}{c}\text { CHO Supplementation } \\
\text { (Brief Description) }\end{array}$ & $\begin{array}{l}\text { SMD IV, Random } \\
{[95 \% \text { CI) }}\end{array}$ \\
\hline $\begin{array}{l}\text { Millard-Stafford et al., } \\
2007 \text { [103] }\end{array}$ & CS & $16 / \mathrm{M}$ & 27.5 & 23.2 & 60.5 & $\mathrm{Lab}$ & $\begin{array}{c}120 \text { min of cycling at fixed } \\
\text { intensities alternating between } \\
60 \% \text { and } 75 \% \dot{\mathrm{VO}}_{2} \text { max every } \\
15 \mathrm{~min}+15 \text { min ride as hard as } \\
\text { possible, TA: } 28.5^{\circ} \mathrm{C}\end{array}$ & $\begin{array}{c}\text { 6\% CHO-E beverage (Gatorade, } \\
\text { Quaker Oats Co, Barrington, IL, } \\
\text { USA), } 6 \mathrm{~mL} \cdot \mathrm{kg}^{-1} 10 \mathrm{~min} \text { before, } \\
6 \mathrm{~mL} \cdot \mathrm{kg}^{-1} \text { at onset of exercise } \\
\text { and } 3 \mathrm{~mL} \cdot \mathrm{kg}^{-1} \text { at } 15 \text {-min } \\
\text { intervals }\end{array}$ & $0.34[-0.36,1.04]$ \\
\hline Morris et al., 2003 [104] & CS & $9 / \mathrm{M}$ & 23.3 & 24.7 & 57.3 & Field & $\begin{array}{l}5 \times 15 \text { min set (walking and } \\
\text { variable speed running) each } \\
\text { separated by } 4 \text { min rest }+60 \mathrm{~s} \\
\text { run and } 60 \mathrm{~s} \text { rest until } \\
\text { exhaustion, TA: } 30.0^{\circ} \mathrm{C}\end{array}$ & $\begin{array}{c}\text { 6.5\% CHO-E solution } \\
\text { (DEX:MD:GL: Lucozade Sport, } \\
\text { SmithKline Beecham), } \\
6.5 \mathrm{~mL} \cdot \mathrm{kg}^{-1} \text { prior to exercise and } \\
4.5 \mathrm{~mL} \cdot \mathrm{kg}^{-1} \text { during every } \\
\text { exercise set and rest period } \\
\text { (19 min) }\end{array}$ & $-0.31[-1.24,0.62]$ \\
\hline Murray et al., 1987 i [105] & CS & $13 / \mathrm{M}$ & 30.6 & 24.6 & 45.1 & $\mathrm{Lab}$ & $\begin{array}{c}\text { intermittent } \mathrm{SS} \text { cycling (at } \\
55 \text { and } 65 \% \mathrm{VO}_{2} \text { max) } \\
\text { interspersed with } 5 \text { rest } \\
\text { periods ( } 3-15 \text { min) and } 2 \text { brief, } \\
\text { high-intensity performance } \\
\text { rides (timed } 240 \text { and } \\
480 \text { revolution cycling task), } \\
\text { TA: } 33.0^{\circ} \mathrm{C}\end{array}$ & $\begin{array}{l}5 \% \mathrm{CHO} \text { drink (GL-polymer), }_{2 \mathrm{~mL} \cdot \mathrm{kg}^{-1} \text { during each of the }} \text { five rest periods }\end{array}$ & $0.63[-0.16,1.42]$ \\
\hline Murray et al., 1987 ii [105] & CS & $13 / \mathrm{M}$ & 30.6 & 24.6 & 45.1 & $\mathrm{Lab}$ & $\begin{array}{c}\text { intermittent } \mathrm{SS} \text { cycling (at } \\
55 \text { and } 65 \% \mathrm{VO}_{2} \text { max) } \\
\text { interspersed with } 5 \text { rest } \\
\text { periods ( } 3-15 \text { min) and } 2 \text { brief, } \\
\text { high-intensity performance } \\
\text { rides (timed } 240 \text { and } \\
480 \text { revolution cycling task), } \\
\text { TA: } 33.0^{\circ} \mathrm{C}\end{array}$ & $\begin{array}{c}6 \% \mathrm{CHO}-\mathrm{E} \text { drink } \\
(4.0 \% \mathrm{SUC}+2.0 \% \mathrm{GL}) \\
2 \mathrm{~mL} \cdot \mathrm{kg}^{-1} \text { during each of the } \\
\text { five rest periods }\end{array}$ & $1.13[0.29,1.97]$ \\
\hline Murray et al., 1987 iii [105] & CS & $13 / \mathrm{M}$ & 30.6 & 24.6 & 45.1 & $\mathrm{Lab}$ & $\begin{array}{c}\text { intermittent } \mathrm{SS} \text { cycling (at } \\
55 \text { and } 65 \% \mathrm{~V}_{2} \text { max) } \\
\text { interspersed with } 5 \text { rest } \\
\text { periods ( } 3-15 \text { min) and } 2 \text { brief, } \\
\text { high-intensity performance } \\
\text { rides (timed } 240 \text { and } \\
480 \text { revolution cycling task), } \\
\text { TA: } 33.0^{\circ} \mathrm{C}\end{array}$ & $\begin{array}{c}7 \% \text { CHO-E drink ( } 5.0 \% \\
\text { GL-polymer }+2.0 \% \text { FRU) } \\
2 \mathrm{~mL} \cdot \mathrm{kg}^{-1} \text { during each of the } \\
\text { five rest periods }\end{array}$ & $1.49[0.60,2.37]$ \\
\hline
\end{tabular}


Table 1. Cont.

\begin{tabular}{|c|c|c|c|c|c|c|c|c|c|}
\hline Study/Trial † & $\begin{array}{l}\text { Study } \\
\text { Design }\end{array}$ & $\begin{array}{c}N / \\
\text { Gender }\end{array}$ & $\begin{array}{l}\text { Age } \\
\text { (year) }\end{array}$ & $\begin{array}{c}\text { BMI } \\
\left(\mathbf{k g} \cdot \mathbf{m}^{-2}\right)\end{array}$ & $\begin{array}{c}\dot{\mathrm{VO}_{2} \max } \\
\left(\mathrm{mL} \cdot \mathrm{kg}^{-1} \cdot \mathrm{min}^{-1}\right)\end{array}$ & $\begin{array}{l}\text { Lab/ } \\
\text { Field }\end{array}$ & $\begin{array}{c}\text { Exercise Task } \\
\text { (Brief Description) }\end{array}$ & $\begin{array}{l}\text { CHO Supplementation } \\
\text { (Brief Description) }\end{array}$ & $\begin{array}{l}\text { SMD IV, Random } \\
{[95 \% \text { CI] }}\end{array}$ \\
\hline Murray et al., 1989 i [106] & CS & $\begin{array}{l}12 / 7 \mathrm{M} \\
5 \mathrm{~F}\end{array}$ & 30.7 & 23.1 & 42.8 & $\mathrm{Lab}$ & $\begin{array}{l}3 \times 20 \text { min of cycling at } 65 \% \\
\dot{\mathrm{V}} \mathrm{O}_{2} \text { max each separated by } \\
5 \text { min rest between each bout }+ \\
\text { time to complete } 1200 \text { pedal } \\
\text { revolutions as fast as possible } \\
\text { (workload: } 65 \% \dot{\mathrm{VO}}_{2} \text { max at } \\
\quad 60 \mathrm{rpm} \text { ), TA: } 33.4^{\circ} \mathrm{C}\end{array}$ & $\begin{array}{l}6 \% \mathrm{CHO}-\mathrm{E} \text { drink (SUC), } 2.5 \mathrm{~mL} \cdot \mathrm{kg}^{-1} \\
\text { prior to exercise and during each of the } \\
\text { three rest periods, served at } 8.0^{\circ} \mathrm{C}\end{array}$ & $0.57[-0.25,1.39]$ \\
\hline Murray et al., 1989 iii [106] & CS & $\begin{array}{l}12 / 7 \mathrm{M} \\
5 \mathrm{~F}\end{array}$ & 30.7 & 23.1 & 42.8 & $\mathrm{Lab}$ & $\begin{array}{c}3 \times 20 \text { min of cycling at } 65 \% \\
\dot{\mathrm{VO}}{ }_{2} \text { max each separated by } \\
5 \text { min rest between each bout }+ \\
\text { time to complete } 1200 \text { pedal } \\
\text { revolutions as fast as possible } \\
\text { (workload: } 65 \% \dot{\mathrm{VO}}_{2} \text { max at } \\
60 \mathrm{rpm} \text { ), } \\
\text { TA: } 33.4{ }^{\circ} \mathrm{C}\end{array}$ & $\begin{array}{l}10 \% \text { CHO-E drink (SUC), } 2.5 \mathrm{~mL} \cdot \mathrm{kg}^{-1} \\
\text { prior to exercise and during each of the } \\
\text { three rest periods, served at } 8.0^{\circ} \mathrm{C}\end{array}$ & $0.05[-0.75,0.85]$ \\
\hline Murray et al., 1991 i [107] & CS & $\begin{array}{l}10 / 8 \mathrm{M} \\
2 \mathrm{~F}\end{array}$ & 32.5 & $\#$ & 48.3 & $\mathrm{Lab}$ & $\begin{array}{c}120 \text { min of cycling at various } \\
\text { intensities }\left(65-75 \% \mathrm{VO}_{2} \text { max }\right)+ \\
4.8 \mathrm{~km} \text { performance, } \\
\text { TA: } 10.0^{\circ} \mathrm{C}\end{array}$ & $\begin{array}{l}6 \% \text { CHO solution (GL, ingestion rate } \\
26 \mathrm{~g} \cdot \mathrm{h}^{-1} \text { : Grain Processing Corp., } \\
\text { Muscatine, IA) at the } 12 \text { th min and every } \\
15 \text { min thereafter, served at } 10.0^{\circ} \mathrm{C}\end{array}$ & $0.60[-0.30,1.50]$ \\
\hline Murray et al., 1991 ii [107] & CS & $\begin{array}{l}10 / 8 \mathrm{M} \\
2 \mathrm{~F}\end{array}$ & 32.5 & $\#$ & 48.3 & $\mathrm{Lab}$ & $\begin{array}{c}120 \text { min of cycling at various } \\
\text { intensities }\left(65-75 \% \dot{\mathrm{VO}}_{2} \max \right)+ \\
4.8 \mathrm{~km} \text { performance, } \\
\text { TA: } 10.0^{\circ} \mathrm{C}\end{array}$ & $\begin{array}{c}12 \% \mathrm{CHO} \text { solution } \\
(8 \% \mathrm{MD}+4 \% \text { DEX, CHO ingestion rate } \\
52 \mathrm{~g} \cdot \mathrm{h}^{-1} \text { : Grain Processing Corp., } \\
\text { Muscatine, IA) at the } 12 \text { th min and every } \\
15 \mathrm{~min} \text { thereafter, served at } 10.0^{\circ} \mathrm{C}\end{array}$ & $0.40[-0.49,1.29]$ \\
\hline Murray et al., 1991 iii [107] & CS & $\begin{array}{l}10 / 8 \mathrm{M} \\
2 \mathrm{~F}\end{array}$ & 32.5 & $\#$ & 48.3 & $\mathrm{Lab}$ & $\begin{array}{c}120 \mathrm{~min} \text { of cycling at various } \\
\text { intensities }\left(65-75 \% \mathrm{VO}_{2} \mathrm{max}\right)+ \\
4.8 \mathrm{~km} \text { performance, } \\
\text { TA: } 10.0^{\circ} \mathrm{C}\end{array}$ & $\begin{array}{c}18 \% \text { CHO solution } \\
(15 \% \mathrm{MD}+3 \% \mathrm{DEX}, \mathrm{CHO} \text { ingestion rate } \\
78 \mathrm{~g} \cdot \mathrm{h}^{-1} \text { : Grain Processing Corp., } \\
\text { Muscatine, IA) at the } 12 \text { th min and every } \\
15 \mathrm{~min} \text { thereafter, served at } 10.0^{\circ} \mathrm{C}\end{array}$ & $0.49[-0.40,1.38]$ \\
\hline
\end{tabular}


Table 1. Cont

\begin{tabular}{|c|c|c|c|c|c|c|c|c|c|}
\hline Study/Trial † & Study Design & $\begin{array}{c}N / \\
\text { Gender }\end{array}$ & $\begin{array}{l}\text { Age } \\
\text { (year) }\end{array}$ & $\begin{array}{c}\text { BMI } \\
\left(\mathrm{kg} \cdot \mathrm{m}^{-2}\right)\end{array}$ & $\begin{array}{c}\dot{\mathrm{VO}_{2} \max } \\
\left(\mathrm{mL} \cdot \mathrm{kg}^{-1} \cdot \mathrm{min}^{-1}\right)\end{array}$ & $\begin{array}{l}\text { Lab/ } \\
\text { Field }\end{array}$ & $\begin{array}{c}\text { Exercise Task } \\
\text { (Brief Description) }\end{array}$ & $\begin{array}{l}\text { CHO Supplementation } \\
\text { (Brief Description) }\end{array}$ & $\begin{array}{l}\text { SMD IV, Random } \\
{[95 \% \text { CI) }}\end{array}$ \\
\hline Naclerio et al., 2014 [108] & CS & $10 / \mathrm{M}$ & 25.0 & 24.0 & $\#$ & $\mathrm{Lab}$ & $\begin{array}{l}90 \text { min of intermittent repeated } \\
\text { running sprint test involving } \\
4 \text { blocks of } 11 \text { sets of } \\
3 \text { repetitions of } 60 \mathrm{~m} \text { at } 60 \% \text {, } \\
80 \% \text {, and } 60 \% \text { maximal aerobic } \\
\text { speed plus } 15 \mathrm{~m} \text { sprint (IRST) }\end{array}$ & $\begin{array}{l}13.9 \% \text { CHO solution, } 500 \mathrm{~mL} \\
\text { containing } 69.5 \mathrm{~g} \text { of } \mathrm{CHO}(\mathrm{MD}) \\
\text { immediately prior to the } 1 \mathrm{st}, 2 \mathrm{nd}, \\
\text { 3rd, and 4th blocks of the IRST }\end{array}$ & $-0.01[-0.89,0.87]$ \\
\hline Nassif et al., 2014 [109] & CS & $10 / \mathrm{M}$ & 26.0 & 22.3 & 70.7 & $\mathrm{Lab}$ & $\begin{array}{c}60 \mathrm{~km} \text { cycling TT punctuated } \\
\text { by } 1-\mathrm{km} \text { sprints }(14,29,44, \\
59 \mathrm{~km}), \mathrm{TA}: 32.0^{\circ} \mathrm{C}\end{array}$ & $\begin{array}{l}6 \% \text { CHO solution }\left(4 \mathrm{~mL} \cdot \mathrm{kg}^{-1} \text { of }\right. \\
\text { BM) at } 5 \mathrm{~km} \text {, after each sprint }(15, \\
30,45 \mathrm{~km}) \text { and at } 55 \mathrm{~km} \text {, served } \\
\text { at } 4.0^{\circ} \mathrm{C}\end{array}$ & $-0.66[-1.56,0.25]$ \\
\hline Nassis et al., 1998 [110] & CS & $\begin{array}{l}9 / 8 \mathrm{M} \\
1 \mathrm{~F}\end{array}$ & 25.0 & 23.5 & 65.1 & $\mathrm{Lab}$ & $\begin{array}{l}15 \mathrm{~s} \text { bouts of fast running (at } \\
80 \% \dot{\mathrm{V}} \mathrm{O}_{2} \text { max for the } 1 \mathrm{st} \\
60 \mathrm{~min} \text {, at } 85 \% \dot{\mathrm{V}} \mathrm{O}_{2} \text { max from } \\
60 \text { to } 100 \text { min of exercise and } \\
\text { finally at } 90 \% \dot{\mathrm{VO}}_{2} \text { max from } \\
100 \text { min of exercise until } \\
\text { exhaustion) separated by } 10 \mathrm{~s} \\
\text { of slow running (at } 45 \% \\
\dot{\mathrm{VO}}_{2} \text { max), } \mathrm{TA}: 21.6^{\circ} \mathrm{C}\end{array}$ & $\begin{array}{l}\text { 6.9\% CHO-E solution served at } \\
8.0-9.0^{\circ} \mathrm{C} \text { (Lucozade-Sport, } \\
\text { SmithKline Beecham Coleford, } \\
\text { Glos, UK) immediately before } \\
\text { the run }\left(3 \mathrm{~mL} \cdot \mathrm{kg}^{-1}\right) \text { and every } \\
20 \mathrm{~min} \text { thereafter }\left(2 \mathrm{~mL} \cdot \mathrm{kg}^{-1}\right)\end{array}$ & $-0.10[-1.02,0.83]$ \\
\hline Newell et al., 2015 i [111] & CS & $20 / \mathrm{M}$ & 34.0 & 23.5 & 62.0 & $\mathrm{Lab}$ & $\begin{array}{c}120 \mathrm{~min} \text { of constant-load ride } \\
\text { at } 95 \% \text { of LT }(185 \pm 25 \mathrm{~W})+\text { a } \\
\text { work-matched TT task } \\
(\sim 30 \text { min at } 70 \% \text { of PPO }) \text {, TA: } \\
19.0^{\circ} \mathrm{C}\end{array}$ & $\begin{array}{l}2.0 \% \text { CHO-E drink } \\
\text { (GL-monomers-polymers) at a } \\
\text { rate of } 1 \mathrm{~L} \cdot \mathrm{h}^{-1}(240 \mathrm{~mL} 2 \mathrm{~min} \\
\text { prior to exercise, subsequently } \\
220 \mathrm{~mL} \text { every } 15 \mathrm{~min} \text { with the } \\
\text { final drink provided at the } 120 \text { th } \\
\text { min of exercise), served at } \\
10.0^{\circ} \mathrm{C}\end{array}$ & $0.34[-0.28,0.97]$ \\
\hline Newell et al., 2015 ii [111] & CS & $20 / \mathrm{M}$ & 34.0 & 23.5 & 62.0 & $\mathrm{Lab}$ & $\begin{array}{c}120 \mathrm{~min} \text { of constant-load ride } \\
\text { at } 95 \% \text { of LT }(185 \pm 25 \mathrm{~W})+\mathrm{a} \\
\text { work-matched TT task } \\
(\sim 30 \text { min at } 70 \% \text { of PPO), TA: } \\
19.0^{\circ} \mathrm{C}\end{array}$ & $\begin{array}{c}3.9 \% \text { CHO-E drink } \\
\text { (GL-monomers-polymers) at a } \\
\text { rate of } 11 \cdot \mathrm{h}^{-1} \text { ( } 240 \mathrm{~mL} 2 \mathrm{~min} \\
\text { prior to exercise, subsequently } \\
220 \mathrm{~mL} \text { every } 15 \mathrm{~min} \text { with the } \\
\text { final drink provided at the } 120 \text { th } \\
\text { min of exercise), served at } \\
10.0^{\circ} \mathrm{C}\end{array}$ & $0.59[-0.05,1.22]$ \\
\hline
\end{tabular}


Table 1. Cont.

\begin{tabular}{|c|c|c|c|c|c|c|c|c|c|}
\hline Study/Trial † & Study Design & $\begin{array}{c}N / \\
\text { Gender }\end{array}$ & $\begin{array}{l}\text { Age } \\
\text { (year) }\end{array}$ & $\begin{array}{c}\text { BMI } \\
\left(\mathbf{k g} \cdot \mathbf{m}^{-2}\right)\end{array}$ & $\begin{array}{l}\mathrm{V}_{2} \mathrm{max}_{2} \\
\left(\mathrm{~mL} \cdot \mathrm{kg}^{-1} \cdot \mathrm{min}^{-1}\right)\end{array}$ & $\begin{array}{l}\text { Lab/ } \\
\text { Field }\end{array}$ & $\begin{array}{c}\text { Exercise Task } \\
\text { (Brief Description) }\end{array}$ & $\begin{array}{l}\text { CHO Supplementation } \\
\text { (Brief Description) }\end{array}$ & $\begin{array}{l}\text { SMD IV, Random } \\
{[95 \% \mathrm{CI})}\end{array}$ \\
\hline Newell et al., 2015 iii [111] & CS & $20 / \mathrm{M}$ & 34.0 & 23.5 & 62.0 & Lab & $\begin{array}{c}120 \mathrm{~min} \text { of constant-load ride } \\
\text { at } 95 \% \text { of } \mathrm{LT}(185 \pm 25 \mathrm{~W})+\mathrm{a} \\
\text { work-matched TT task } \\
(\sim 30 \mathrm{~min} \text { at } 70 \% \text { of PPO), TA: } \\
19.0^{\circ} \mathrm{C}\end{array}$ & $\begin{array}{l}6.4 \% \text { CHO-E drink } \\
\text { (GL-monomers-polymers) at a rate } \\
\text { of } 1 \mathrm{~L} \cdot \mathrm{h}^{-1}(240 \mathrm{~mL} 2 \mathrm{~min} \text { prior to } \\
\text { exercise, subsequently } 220 \mathrm{~mL} \\
\text { every } 15 \mathrm{~min} \text { with the final drink } \\
\text { provided at the } 120 \text { th min of } \\
\text { exercise), served at } 10.0^{\circ} \mathrm{C}\end{array}$ & $0.62[-0.02,1.25]$ \\
\hline Nicholas et al., 1995 [112] & CS & $9 / \mathrm{M}$ & 24.8 & 24.9 & 56.3 & Field & $\begin{array}{c}5 \times 15 \text { min periods of } \\
\text { intermittent running, } \\
\text { consisting of sprinting, } \\
\text { interspersed with periods of } \\
\text { jogging and walking }+ \\
\text { intermittent running to fatigue } \\
\text { (corresponding to } 55 \text { and } 95 \% \\
\mathrm{VO}_{2} \text { max, alternating every } \\
20 \text { m), TA: } 20.0^{\circ} \mathrm{C} \\
\end{array}$ & $\begin{array}{c}\text { 6.9\% CHO-E solution } \\
\text { (Lucozade-Sport, SmithKline } \\
\text { Beecham, Brentford, UK) } \\
\text { immediately prior to exercise } \\
\left(5 \mathrm{~mL} \cdot \mathrm{kg}^{-1}\right) \text { and every } 15 \mathrm{~min} \\
\text { thereafter }\left(2 \mathrm{~mL} \cdot \mathrm{kg}^{-1}\right)\end{array}$ & $0.55[-0.40,1.49]$ \\
\hline $\begin{array}{l}\text { Nikolopoulos et al., } \\
2004 \text { [113] }\end{array}$ & CS & $8 / \mathrm{M}$ & 25.0 & $\#$ & 66.0 & $\mathrm{Lab}$ & TE cycling at $84 \pm 1 \% \dot{\mathrm{VO}}_{2} \max$ & $\begin{array}{l}6.4 \% \text { CHO-E solution, } 10 \mathrm{~min} \text { prior } \\
\text { to exercise }\left(8 \mathrm{~mL} \cdot \mathrm{kg}^{-1}\right) \text { and every } \\
15 \mathrm{~min} \text { thereafter }\left(2 \mathrm{~mL} \cdot \mathrm{kg}^{-1}\right)\end{array}$ & $0.34[-0.65,1.33]$ \\
\hline Nishibata et al., 1993 [114] & CS & $7 / \mathrm{M}$ & 27.0 & 22.5 & 50.3 & $\mathrm{Lab}$ & $\begin{array}{c}\text { TE cycling at } 73.4 \pm 7.7 \% \\
\dot{\mathrm{VO}}_{2} \max \end{array}$ & $\begin{array}{l}10 \% \text { GL-drink } 15 \text { min prior to } \\
\text { exercise and at the } 15 \text { th min and } \\
\text { 45th min of exercise equal amounts, } \\
\text { chilled with ice (total: } \\
\text { GL } 43.1 \pm 4.2 \mathrm{~g} \text { ) }\end{array}$ & $-0.20[-1.25,0.85]$ \\
\hline $\begin{array}{l}\text { Oosthuyse et al., } \\
\text { 2015a i [115] }\end{array}$ & CS & $9 / \mathrm{M}$ & 38.0 & 24.2 & 60.8 & $\mathrm{Lab}$ & $\begin{array}{c}120 \text { min of SS cycling }(60 \% \\
\text { Wmax) + a } 16 \mathrm{~km} \mathrm{TT}, \mathrm{TA}: \\
17.0-19.0^{\circ} \mathrm{C}\end{array}$ & $\begin{array}{c}7 \% \text { CHO-E solution equal to } \\
900 \mathrm{~mL} \cdot \mathrm{h}^{-1} \text { and CHO ingestion } \\
\text { rate } 63 \mathrm{~g} \cdot \mathrm{h}^{-1} \text { of } 0.8: 1 \text { FRU:MD } \\
\text { (Krystar } 300 \text { crystalline FRU, Tate \& } \\
\text { Lyle, Decatur, IL, USA \& Glucidex } \\
\text { 12, Roquette Frères, Lestrem Cedex, } \\
\text { France) }\end{array}$ & $0.07[-0.86,0.99]$ \\
\hline $\begin{array}{l}\text { Oosthuyse et al., } \\
\text { 2015a ii [115] }\end{array}$ & CS & $9 / \mathrm{M}$ & 38.0 & 24.2 & 60.8 & Lab & $\begin{array}{c}120 \text { min of SS cycling }(60 \% \\
\text { Wmax }+ \text { a } 16 \text { km TT, TA: } \\
17.0-19.0^{\circ} \mathrm{C}\end{array}$ & $\begin{array}{c}7 \% \text { CHO-E solution equal to } \\
900 \mathrm{~mL} \cdot \mathrm{h}^{-1} \text { and CHO ingestion } \\
\text { rate } 63 \mathrm{~g} \cdot \mathrm{h}^{-1} \text { of IsoMAL } \\
\text { (Palatinose, Beneo-Palatinit GmbH, } \\
\text { Mannheim, Germany) }\end{array}$ & $-0.31[-1.24,0.62]$ \\
\hline
\end{tabular}


Table 1. Cont.

\begin{tabular}{|c|c|c|c|c|c|c|c|c|c|}
\hline Study/Trial † & Study Design & $\begin{array}{l}N / \\
\text { Gender }\end{array}$ & $\begin{array}{l}\text { Age } \\
\text { (year) }\end{array}$ & $\begin{array}{c}\text { BMI } \\
\left(\mathbf{k g} \cdot \mathrm{m}^{-2}\right)\end{array}$ & 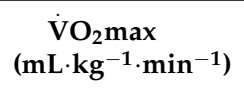 & $\begin{array}{l}\text { Lab/ } \\
\text { Field }\end{array}$ & $\begin{array}{c}\text { Exercise Task } \\
\text { (Brief Description) }\end{array}$ & $\begin{array}{l}\text { CHO Supplementation } \\
\text { (Brief Description) }\end{array}$ & $\begin{array}{l}\text { SMD IV, Random } \\
{[95 \% \text { CI] }}\end{array}$ \\
\hline $\begin{array}{c}\text { Oosthuyse et al., 2015b } \\
\text { [116] }\end{array}$ & CS & $8 / \mathrm{M}$ & 38.9 & 24.3 & 60.9 & $\mathrm{Lab}$ & $\begin{array}{c}120 \text { min of SS cycling }(60 \% \\
\text { Wmax })+ \text { a } 16 \mathrm{~km} \mathrm{TT} \\
\text { TA: } 19.2^{\circ} \mathrm{C}\end{array}$ & $\begin{array}{l}7 \% \text { CHO-E solution equal to } \\
900 \mathrm{~mL} \cdot \mathrm{h}^{-1} \text { and CHO ingestion rate } \\
63 \mathrm{~g} \cdot \mathrm{h}^{-1} \text { at a ratio of } 0.8: 1 \mathrm{FRU}: \mathrm{MD} \\
\text { (Krystar } 300 \text { crystalline FRU, Tate \& } \\
\text { Lyle, Decatur, IL, USA \& Glucidex } 12 \text {, } \\
\text { Roquette Frères, Lestrem Cedex, } \\
\text { France), } 400 \mathrm{~mL} \text { before commencing } \\
\text { exercise, } 200 \mathrm{~mL} \text { at every } 15 \mathrm{~min}\end{array}$ & $0.15[-0.83,1.14]$ \\
\hline Osterberg et al., 2008 [117] & CS & $13 / \mathrm{M}$ & 31.2 & 22.6 & $56.0 *$ & $\mathrm{Lab}$ & $\begin{array}{c}120 \mathrm{~min} \text { of constant-load } \\
\text { cycling at a workload } 5 \% \\
\text { below OBLA followed by a TT } \\
\text { to complete a set amount of } \\
\text { work }\left(7 \mathrm{~kJ} \cdot \mathrm{kg}^{-1}\right) \text { as quickly as } \\
\text { possible, TA: } 23.0^{\circ} \mathrm{C}\end{array}$ & $\begin{array}{c}\text { 6\% CHO-E beverage } \\
\text { (2\% SUC + } 2 \% \text { GL + } 2 \% \text { FRU: } \\
\text { Gatorade Thirst Quencher, The } \\
\text { Gatorade Company, Chicago, IL, } \\
\text { USA), } 250 \text { mL every } 15 \text { min during } \\
\text { the constant-load ride }\end{array}$ & $0.60[-0.19,1.39]$ \\
\hline O'Hara et al., 2014 i [118] & CS & $10 / \mathrm{M}$ & 31.0 & $\#$ & 58.1 & Lab & $\begin{array}{c}4 \times 5 \text { min of continuous } \\
\text { progressive workload } \\
\text { increments corresponding to } \\
70,75,80 \text {, and } 85 \% \text { Wmax }+10 \\
\times 90 \text { s sprints at } 90 \% \text { Wmax } \\
\text { separated by } 180 \text { s recovery at } \\
55 \% \text { Wmax }+ \text { cycling to } \\
\text { volitional exhaustion at } 90 \% \\
\text { Wmax, TA: } 18.0^{\circ} \mathrm{C}\end{array}$ & $\begin{array}{l}\text { 4\% CHO solution, } 40 \mathrm{~g} \text { of GAL } \\
\text { (D-GAL: Inalco, Milan, Italy) as } 11 \\
\text { formulation }\end{array}$ & $0.34[-0.54,1.22]$ \\
\hline O'Hara et al., 2014 ii [118] & CS & $10 / \mathrm{M}$ & 31.0 & $\#$ & 58.1 & $\mathrm{Lab}$ & $\begin{array}{c}4 \times 5 \text { min of continuous } \\
\text { progressive workload } \\
\text { increments corresponding to } \\
70,75,80 \text {, and } 85 \% \text { Wmax }+10 \\
\times 90 \mathrm{~s} \text { sprints at } 90 \% \text { Wmax } \\
\text { separated by } 180 \text { s recovery at } \\
55 \% \text { Wmax }+ \text { cycling to } \\
\text { volitional exhaustion at } 90 \% \\
\text { Wmax, TA: } 18.0^{\circ} \mathrm{C}\end{array}$ & $\begin{array}{l}\text { 4\% CHO solution, } 40 \mathrm{~g} \text { of GL (D-GL: } \\
\text { Cargill, Manchester, UK) as } 11 \\
\text { formulation }\end{array}$ & $\begin{array}{c}-0.25[-1.13 \\
0.63]\end{array}$ \\
\hline O'Neal et al., 2013 [119] & CS & $\begin{array}{l}36 / 23 \mathrm{M} \\
13 \mathrm{~F}\end{array}$ & 23.0 & 23.8 & $\#$ & Lab & $\begin{array}{c}50 \mathrm{~min} \text { of stationary cycling at } \\
\sim 60-65 \% \text { of heart rate reserve } \\
(146 \pm 4 \mathrm{bpm}) \text { interspersed } \\
\text { with } 5 \text { rest periods of } 2 \mathrm{~min} \\
\text { each }+3 \times 30 \mathrm{~s} \text { WAT with } \\
2.5 \text { min rest between tests, TA: } \\
25.0^{\circ} \mathrm{C}\end{array}$ & $\begin{array}{l}6 \% \text { CHO-E beverage in } 3 \text { equal } \\
\text { aliquots, at minutes } 0,20 \text {, and } \\
40 \text { during the } 60 \text {-min submaximal } \\
\text { cycling bout (mean total beverage } 847 \\
\pm 368 \text { mL: equivalent to participant's } \\
\text { sweat losses), served chilled }\end{array}$ & $0.10[-0.36,0.56]$ \\
\hline
\end{tabular}


Table 1. Cont.

\begin{tabular}{|c|c|c|c|c|c|c|c|c|c|}
\hline Study/Trial + & Study Design & $\begin{array}{c}N / \\
\text { Gender }\end{array}$ & $\begin{array}{c}\text { Age } \\
\text { (year) }\end{array}$ & $\begin{array}{c}\text { BMI } \\
\left(\mathrm{kg} \cdot \mathrm{m}^{-2}\right)\end{array}$ & $\begin{array}{c}\dot{\mathrm{VO}}_{2} \max \\
\left(\mathrm{mL} \cdot \mathrm{kg}^{-1} \cdot \mathrm{min}^{-1}\right)\end{array}$ & $\begin{array}{l}\text { Lab/ } \\
\text { Field }\end{array}$ & $\begin{array}{c}\text { Exercise Task } \\
\text { (Brief Description) }\end{array}$ & $\begin{array}{l}\text { CHO Supplementation } \\
\text { (Brief Description) }\end{array}$ & $\begin{array}{l}\text { SMD IV, Random } \\
{[95 \% \mathrm{CI})}\end{array}$ \\
\hline Pettersson et al., 2019 [120] & CS & $\begin{array}{l}12 / 6 \mathrm{M} \\
6 \mathrm{~F}\end{array}$ & $\begin{array}{l}25.6 \mathrm{M} \\
\text { and } \\
24.8 \mathrm{~F}\end{array}$ & $\#$ & $\begin{array}{l}69.1 * \mathrm{M} \text { and } \\
59.9 * \mathrm{~F}\end{array}$ & Lab & $\begin{array}{c}120 \text { min of submaximal } \\
\text { diagonal-style roller-skiing } \\
\left(69.3 \pm 2.9 \% \text { of } \dot{\mathrm{VO}}_{2} \text { peak) at a }\right. \\
\text { constant incline of } 5^{\circ} \\
\text { (treadmill speed, males: } \\
9.7 \pm 0.2 \mathrm{~km} \cdot \mathrm{h}^{-1} \text {, females: } \\
\left.8.5 \pm 0.3 \mathrm{~km} \cdot \mathrm{h}^{-1}\right)+ \text { final } \\
2000 \mathrm{~m} \text { for females or } 2400 \mathrm{~m} \\
\text { for males TT, TA: }-5.0^{\circ} \mathrm{C}\end{array}$ & $\begin{array}{l}18 \% \text { CHO-E solution (MD:FRU, in a } \\
\text { ratio of 1:0.8) with additional gelling } \\
\text { polysaccharides (Maurten AB, } \\
\text { Gothenburg, Sweden), } 222 \mathrm{~mL} \\
\text { before the onset of exercise, } \\
\text { subsequently, } 220 \mathrm{~mL} \text { every } 20 \mathrm{~min}, \\
\text { CHO ingestion rate } 132 \mathrm{~g} \cdot \mathrm{h}^{-1}\end{array}$ & $\begin{array}{c}-0.06[-0.86 \\
0.74]\end{array}$ \\
\hline Pottier et al., 2010 [121] & CS & $12 / \#$ & 30.2 & $\#$ & 61.7 & Lab & $\begin{array}{c}\text { amount of cycling work }(0.75 \\
\times \text { Wmax } \times 3600) \text { as fast as } \\
\text { possible }(\sim 60 \text { min of cycling }) \\
\text { TA: } 19.0-21.0^{\circ} \mathrm{C}\end{array}$ & $\begin{array}{c}5.86 \% \text { CHO-E solution ( } 5.4 \% \text { SUC + } \\
0.46 \% \text { GL: Gatorade), before and after } \\
\text { warm-up, } 2 \text { and } 1.5 \mathrm{~mL} \cdot \mathrm{kg}^{-1} \mathrm{BM} \\
\text { respectively and after reaching each } \\
12.5 \% \text { of the total amount of work; } \\
\text { subjects also received } 1.5 \mathrm{~mL} \cdot \mathrm{kg}^{-1} \\
\text { BM }\end{array}$ & $\begin{array}{c}-0.16[-0.96 \\
0.64]\end{array}$ \\
\hline Rilley et al., 1988 [122] & CS & $9 / \mathrm{M}$ & 30.0 & 22.9 & 65.0 & Lab & TE cycling at $70-75 \% \dot{\mathrm{VO}}_{2} \max$ & $\begin{array}{l}\text { 7\% CHO drink (5\% GL-polymer + } 2 \% \\
\text { FRU: Exceed, Ross Laboratories, } \\
\text { Columbus, OH) at } 20 \text {-min intervals } \\
\text { beginning } 20 \text { min prior to exercise } \\
\text { (14 g CHO each treatment) }\end{array}$ & $0.16[-0.77,1.08]$ \\
\hline Roberts et al., 2014 i [123] & CS & $14 / \mathrm{M}$ & 31.8 & 23.0 & 60.4 & Lab & $\begin{array}{c}150 \text { min of continuous cycling } \\
\text { at } 50 \% \text { Wmax }(176.71 \pm \\
25.92 \mathrm{~W})+ \text { a } 60 \mathrm{~km} \text { cycling TT, } \\
\text { TA: } 22.4^{\circ} \mathrm{C}\end{array}$ & $\begin{array}{l}10 \% \text { CHO-E beverage (High } 5 \mathrm{Ltd} .) \\
\text { MD } 1.1 \mathrm{~g} \cdot \mathrm{min}^{-1}+\text { FRU } 0.6 \mathrm{~g} \cdot \mathrm{min}^{-1} \\
270 \mathrm{~mL} \text { doses at the start and every } \\
15 \mathrm{~min} \text { (until completion of the } \\
\text { performance trial) }\end{array}$ & $0.50[-0.47,1.48]$ \\
\hline Roberts et al., 2014 ii [123] & CS & $14 / \mathrm{M}$ & 31.8 & 23.0 & 60.4 & Lab & $\begin{array}{c}150 \text { min of continuous cycling } \\
\text { at } 50 \% \text { Wmax }(176.71 \pm \\
25.92 \mathrm{~W})+ \text { a } 60 \mathrm{~km} \text { cycling TT, } \\
\text { TA: } 22.4^{\circ} \mathrm{C}\end{array}$ & $\begin{array}{l}10 \% \text { CHO-E beverage (High } 5 \text { Ltd.), } \\
\text { MD } 1.7 \mathrm{~g} \cdot \mathrm{min}^{-1}, 270 \mathrm{~mL} \text { doses at the } \\
\text { start and every } 15 \mathrm{~min} \text { (until } \\
\text { completion of the performance trial) }\end{array}$ & $\begin{array}{c}-0.06[-1.02 \\
0.90]\end{array}$ \\
\hline $\begin{array}{l}\text { Robson-Ansley et al., } \\
2009[124]\end{array}$ & CS & $7 / \mathrm{M}$ & 24.0 & 23.1 & $\#$ & Lab & $\begin{array}{l}90 \text { min run of self-paced TT on } \\
\text { a motorized treadmill }\end{array}$ & $\begin{array}{l}8 \% \text { CHO fluid, } 8 \mathrm{~mL} \cdot \mathrm{kg}^{-1} 5 \mathrm{~min} \\
\text { prior to exercise and } 2 \mathrm{~mL} \cdot \mathrm{kg}^{-1} \\
\quad \text { every } 20 \mathrm{~min} \text { thereafter }\end{array}$ & $0.44[-0.63,1.50]$ \\
\hline $\begin{array}{l}\text { Robson-Ansley et al., } \\
2011 \text { [125] }\end{array}$ & CS & $9 / \mathrm{M}$ & 26.0 & 22.4 & 58.0 & Lab & $\begin{array}{c}120 \text { min of continuous running } \\
\text { at velocity } \sim 60 \% \dot{\mathrm{VO}}_{2} \max \\
\text { followed by a } 5 \mathrm{~km} \mathrm{TT} \text {, TA: } \\
20.0^{\circ} \mathrm{C}\end{array}$ & $\begin{array}{l}8 \% \text { CHO solution immediately before } \\
\text { and at } 20 \text {-min intervals during the } \\
\text { preload bout }\left(2 \mathrm{~mL} \cdot \mathrm{kg}^{-1} \mathrm{BM}\right)\end{array}$ & $0.16[-0.76,1.09]$ \\
\hline
\end{tabular}


Table 1. Cont.

\begin{tabular}{|c|c|c|c|c|c|c|c|c|c|}
\hline Study/Trial + & Study Design & $\begin{array}{c}N / \\
\text { Gender }\end{array}$ & $\begin{array}{c}\text { Age } \\
\text { (year) }\end{array}$ & $\begin{array}{c}\text { BMI } \\
\left(\mathbf{k g} \cdot \mathrm{m}^{-2}\right)\end{array}$ & $\begin{array}{c}\mathrm{VO}_{2} \max \\
\left(\mathrm{mL} \cdot \mathrm{kg}^{-1} \cdot \mathrm{min}^{-1}\right)\end{array}$ & $\begin{array}{l}\text { Lab/ } \\
\text { Field }\end{array}$ & $\begin{array}{c}\text { Exercise Task } \\
\text { (Brief Description) }\end{array}$ & $\begin{array}{l}\text { CHO Supplementation } \\
\text { (Brief Description) }\end{array}$ & $\begin{array}{l}\text { SMD IV, Random } \\
{[95 \% \mathrm{CI})}\end{array}$ \\
\hline $\begin{array}{c}\text { Rollo and Williams } \\
2009 \text { [126] }\end{array}$ & CS & $8 / \mathrm{M}$ & 31.0 & 23.1 & $62.0 *$ & $\mathrm{Lab}$ & $\begin{array}{c}60 \text { min performance run, TA: } \\
16^{\circ} \mathrm{C}\end{array}$ & $\begin{array}{l}\text { 6.4\% CHO-E solution (Lucozade } \\
\text { Sport, Brentford, UK), } 30 \mathrm{~min} \\
\text { before the 1-hr run }\left(8 \mathrm{~mL} \cdot \mathrm{kg}^{-1}\right. \\
\mathrm{BM} \text { ) and at } 15-\mathrm{min} \text { intervals } \\
\text { during the run } 2 \mathrm{~mL} \cdot \mathrm{kg}^{-1} \mathrm{BM} \\
\text { (total: } \mathrm{CHO} \text { ingestion rate } \\
\left.\sim 60 \mathrm{~g} \cdot \mathrm{h}^{-1}\right)\end{array}$ & $0.28[-0.71,1.26]$ \\
\hline $\begin{array}{l}\text { Rollo and Williams } \\
2010 \text { [127] }\end{array}$ & CS & $10 / \mathrm{M}$ & 34.0 & 23.0 & $62.0 *$ & $\mathrm{Lab}$ & $\begin{array}{l}60 \text { min run as far as possible on } \\
\text { an automated treadmill that } \\
\text { allowed changes in running } \\
\text { speed without manual input, } \\
\text { TA: } 16.0^{\circ} \mathrm{C}\end{array}$ & $\begin{array}{c}\text { 6.4\% CHO-E solution }\left(8 \mathrm{~mL} \cdot \mathrm{kg}^{-1}\right. \\
\mathrm{BM}, \text { Lucozade Sport, Brentford, } \\
\text { UK), } 30 \text { min before and } \\
2 \mathrm{~mL} \cdot \mathrm{kg}^{-1} \mathrm{BM} \text { at } 15 \text {-min } \\
\text { intervals }\end{array}$ & $\begin{array}{c}-0.06[-0.93 \\
0.82]\end{array}$ \\
\hline Rollo et al., 2011 [128] & CS & $10 / \mathrm{M}$ & 26.0 & 22.6 & $65.0 *$ & $\mathrm{Lab}$ & $\begin{array}{l}60 \text { min performance run, TA: } \\
20^{\circ} \mathrm{C}\end{array}$ & $\begin{array}{l}\text { 6.4\% CHO-E solution (Lucozade } \\
\text { Sport, Brentford, UK), } 30 \mathrm{~min} \\
\text { before the } 1 \text {-hr run }\left(8 \mathrm{~mL} \cdot \mathrm{kg}^{-1}\right. \\
\mathrm{BM} \text { ) and at } 15-\mathrm{min} \text { intervals } \\
\text { during the run } 2 \mathrm{~mL} \cdot \mathrm{kg}^{-1} \mathrm{BM} \\
\text { (total: } \mathrm{CHO} \text { ingestion rate } \\
\left.\sim 60 \mathrm{~g} \cdot \mathrm{h}^{-1}\right)\end{array}$ & $0.40[-0.49,1.29]$ \\
\hline Smith et al., 2010 ii [129] & CS & $12 / \mathrm{M}$ & 31.7 & 23.4 & $55.3 *$ & $\mathrm{Lab}$ & $\begin{array}{c}120 \text { min of constant-load ride } \\
\left(77 \% \mathrm{VO}_{2} \text { peak }\right)+\text { a } 20 \mathrm{~km} \mathrm{TT}, \\
\text { TA: } 23.0^{\circ} \mathrm{C}\end{array}$ & $\begin{array}{l}3.0 \% \text { CHO-E solution (GL, } \\
\left.\text { ingestion rate } 30 \mathrm{~g} \cdot \mathrm{h}^{-1}\right), 2000 \mathrm{~mL} \\
(250 \mathrm{~mL} \text { every } 15 \mathrm{~min}, \text { starting at } \\
\text { min } 15 \text { and ending at min } 120)\end{array}$ & $0.49[-0.32,1.31]$ \\
\hline Smith et al., 2010 iii [129] & CS & $12 / \mathrm{M}$ & 31.7 & 23.4 & $55.3 *$ & $\mathrm{Lab}$ & $\begin{array}{c}120 \text { min of constant-load ride } \\
\left(77 \% \mathrm{VO}_{2} \text { peak }\right)+\text { a } 20 \mathrm{~km} \mathrm{TT} \\
\text { TA: } 23.0^{\circ} \mathrm{C}\end{array}$ & $\begin{array}{l}\text { 6.0\% CHO-E solution (GL, } \\
\left.\text { ingestion rate } 60 \mathrm{~g} \cdot \mathrm{h}^{-1}\right), 2000 \mathrm{~mL} \\
(250 \mathrm{~mL} \text { every } 15 \mathrm{~min} \text {, starting at } \\
\text { min } 15 \text { and ending at min } 120)\end{array}$ & $0.65[-0.18,1.47]$ \\
\hline Steiner et al., 2009 [130] & CS & $\begin{array}{l}9 / 6 \mathrm{M} \\
3 \mathrm{~F}\end{array}$ & $\begin{array}{l}28.2 \mathrm{M} \\
21.5 \mathrm{~F}\end{array}$ & 22.6 & $45.7^{*}$ & Lab & $\begin{array}{c}\text { cycling } 45 \text { min production trial } \\
\text { consisting of a } 5 \text {-min warm-up } \\
\text { at } 50 \mathrm{~W}+\text { by } 45 \mathrm{~min} \text { of } \\
\text { self-selected PO at an RPE of } \\
16 \text { (at } 70-80 \mathrm{rpm})\end{array}$ & $\begin{array}{l}\text { 6\% CHO-E solution (Gatorade } \\
\text { Sports Science Institute, } \\
\text { Barrington, IL, USA), } 240 \mathrm{~mL} \text { just } \\
\text { prior to exercise and every } \\
15 \text { min during the production } \\
\text { trial }\end{array}$ & $0.07[-0.85,1.00]$ \\
\hline
\end{tabular}


Table 1. Cont.

\begin{tabular}{|c|c|c|c|c|c|c|c|c|c|}
\hline Study/Trial + & Study Design & $\begin{array}{l}N / \\
\text { Gender }\end{array}$ & $\begin{array}{l}\text { Age } \\
\text { (year) }\end{array}$ & $\begin{array}{c}\text { BMI } \\
\left(\mathbf{k g} \cdot \mathrm{m}^{-2}\right)\end{array}$ & $\begin{array}{c}\mathrm{VO}_{2} \max \\
\left(\mathrm{mL} \cdot \mathrm{kg}^{-1} \cdot \mathrm{min}^{-1}\right)\end{array}$ & $\begin{array}{l}\text { Lab/ } \\
\text { Field }\end{array}$ & $\begin{array}{c}\text { Exercise Task } \\
\text { (Brief Description) }\end{array}$ & $\begin{array}{l}\text { CHO Supplementation } \\
\text { (Brief Description) }\end{array}$ & $\begin{array}{l}\text { SMD IV, Random } \\
{[95 \% \text { CI }]}\end{array}$ \\
\hline Sun et al., 2015 [131] & CS & $8 / \mathrm{F}$ & 28.3 & 19.8 & 48.3 & $\mathrm{Lab}$ & $\begin{array}{c}\text { running to exhaustion at } 70 \% \\
\mathrm{~V}_{2} \text { max on a treadmill, } \mathrm{TA} \text { : } \\
20.3-21.3^{\circ} \mathrm{C}\end{array}$ & $\begin{array}{l}\text { 6\% CHO-E solution }\left(3 \mathrm{~mL} \cdot \mathrm{kg}^{-1}\right. \\
\mathrm{BM}) \text { every } 20 \mathrm{~min} \text { during } \\
\text { exercise }\end{array}$ & $0.62[-0.39,1.63]$ \\
\hline $\begin{array}{c}\text { Tokmakidis \& } \\
\text { Karamanolis } 2008 \text { [132] }\end{array}$ & CS & $\begin{array}{c}11 / 10 \mathrm{M} \\
1 \mathrm{~F}\end{array}$ & 25.3 & 24.5 & 49.0 & $\mathrm{Lab}$ & $\begin{array}{c}\text { running at } 60 \% \dot{\mathrm{VO}}_{2} \text { max for } \\
5 \mathrm{~min}, \text { at } 70 \% \text { for } 45 \mathrm{~min}+\text { at } \\
80 \% \dot{\mathrm{VO}}_{2} \text { max until exhaustion }\end{array}$ & $\begin{array}{c}\sim 18.5 \% \text { CHO solution (GL, } \\
\left.1 \mathrm{~g} \cdot \mathrm{kg}^{-1}, 400 \mathrm{~g}\right), 15 \mathrm{~min} \text { prior to } \\
\text { exercise }\end{array}$ & $0.27[-0.57,1.11]$ \\
\hline Tsintzas et al., 1993 [133] & CS & $\begin{array}{l}7 / 4 \mathrm{M} \\
3 \mathrm{~F}\end{array}$ & 32.6 & 22.4 & 61.9 & Field & $30 \mathrm{~km}$ race running & $\begin{array}{c}5 \% \text { CHO solution } \\
\text { (1.8\% GL-polymer + } 2 \% \text { FRU }+1.2 \% \\
\text { other CHO: Replay, Bass Ltd.), } \\
250 \mathrm{~mL} \text { at onset of exercise and } \\
150 \mathrm{~mL} \text { every } 5 \mathrm{~km} \text { thereafter }\end{array}$ & $0.14[-0.91,1.19]$ \\
\hline Tsintzas et al., 1995 i [134] & CS & $7 / \mathrm{M}$ & 44.0 & 21.3 & 58.4 & $\mathrm{Lab}$ & $\begin{array}{l}\text { marathon-race running, TA: } \\
\qquad 20.0^{\circ} \mathrm{C}\end{array}$ & $\begin{array}{c}6.9 \% \text { CHO-E drink } \\
(3.1 \% \mathrm{MD}+3.8 \% \mathrm{SUC}) \\
3 \mathrm{~mL} \cdot \mathrm{kg}^{-1} \text { prior to exercise and } \\
2 \mathrm{~mL} \cdot \mathrm{kg}^{-1} \text { every } 5 \mathrm{~km} \text { thereafter }\end{array}$ & $0.13[-0.92,1.17]$ \\
\hline Tsintzas et al., 1995 ii [134] & CS & $7 / \mathrm{M}$ & 44.0 & 21.3 & 58.4 & $\mathrm{Lab}$ & $\begin{array}{l}\text { marathon-race running, TA: } \\
\qquad 20.0^{\circ} \mathrm{C}\end{array}$ & $\begin{array}{c}5.5 \% \text { CHO-E drink } \\
(2.7 \% \mathrm{MD}+1.7 \% \mathrm{GL}-\mathrm{syrup}+ \\
1.1 \% \mathrm{FRU}-\mathrm{syrup}), 3 \mathrm{~mL} \cdot \mathrm{kg}^{-1} \\
\text { prior to exercise and } 2 \mathrm{~mL} \cdot \mathrm{kg}^{-1} \\
\text { every } 5 \mathrm{~km} \text { thereafter }\end{array}$ & $0.31[-0.75,1.36]$ \\
\hline Tsintzas et al., 1996a [135] & CS & $8 / \mathrm{M}$ & 29.7 & 23.4 & 61.8 & $\mathrm{Lab}$ & $\begin{array}{c}\text { TE running at } 70 \% \dot{\mathrm{VO}}_{2} \max , \\
\text { TA: } 19.8{ }^{\circ} \mathrm{C}\end{array}$ & $\begin{array}{c}5.5 \% \text { CHO-E solution } \\
(1.7 \% \mathrm{GL}+1.1 \% \text { FRU }+0.6 \% \\
\mathrm{MAL}+2.1 \% \text { saccharides }) \\
8 \mathrm{~mL} \cdot \mathrm{kg}^{-1} \text { prior to exercise and } \\
2 \mathrm{~mL} \cdot \mathrm{kg}^{-1} \text { every } 20 \mathrm{~min} \\
\text { thereafter }\end{array}$ & $0.89[-0.16,1.93]$ \\
\hline Tsintzas et al., 1996b i [136] & CS & $11 / \mathrm{M}$ & 27.0 & 23.3 & 61.7 & $\mathrm{Lab}$ & $\begin{array}{c}\text { TE running at } 70 \% \dot{\mathrm{VO}}_{2} \max , \\
\text { TA: } 19.5^{\circ} \mathrm{C}\end{array}$ & $\begin{array}{c}5.5 \% \text { CHO-E solution } \\
(1.7 \% \mathrm{GL}+1.1 \% \text { FRU }+0.6 \% \\
\mathrm{MAL}+2.1 \% \text { saccharides }) \\
8 \mathrm{~mL} \cdot \mathrm{kg}^{-1} \text { prior to exercise and } \\
2 \mathrm{~mL} \cdot \mathrm{kg}^{-1} \text { every } 20 \mathrm{~min} \\
\text { thereafter }\end{array}$ & $0.49[-0.36,1.34]$ \\
\hline Tsintzas et al., 1996b ii [136] & CS & $11 / \mathrm{M}$ & 27.0 & 23.3 & 61.7 & $\mathrm{Lab}$ & $\begin{array}{c}\text { TE running at } 70 \% \dot{\mathrm{VO}}_{2} \max \\
\text { TA: } 19.5^{\circ} \mathrm{C}\end{array}$ & $\begin{array}{c}\text { 6.9\% CHO-E drink (Lucozade } \\
\text { Sport, SmithKline Beecham, UK), } \\
8 \mathrm{~mL} \cdot \mathrm{kg}^{-1} \text { prior to exercise and } \\
2 \mathrm{~mL} \cdot \mathrm{kg}^{-1} \text { every } 20 \mathrm{~min} \\
\text { thereafter }\end{array}$ & $0.37[-0.48,1.21]$ \\
\hline
\end{tabular}


Table 1. Cont.

\begin{tabular}{|c|c|c|c|c|c|c|c|c|c|}
\hline Study/Trial + & Study Design & $\begin{array}{l}N / \\
\text { Gender }\end{array}$ & $\begin{array}{c}\text { Age } \\
\text { (year) }\end{array}$ & $\begin{array}{c}\text { BMI } \\
\left(\mathbf{k g} \cdot \mathrm{m}^{-2}\right)\end{array}$ & $\begin{array}{l}\dot{\mathrm{VO}}_{2} \mathrm{max} \\
\left(\mathrm{mL} \cdot \mathrm{kg}^{-1} \cdot \mathrm{min}^{-1}\right)\end{array}$ & $\begin{array}{l}\text { Lab/ } \\
\text { Field }\end{array}$ & $\begin{array}{c}\text { Exercise Task } \\
\text { (Brief Description) }\end{array}$ & $\begin{array}{l}\text { CHO Supplementation } \\
\text { (Brief Description) }\end{array}$ & $\begin{array}{l}\text { SMD IV, Random } \\
{[95 \% \mathrm{CI})}\end{array}$ \\
\hline Utter et al., 2002 [137] & PS & $\begin{array}{l}48 / \# \mathrm{M} \\
\quad \# \mathrm{~F}\end{array}$ & $41.2-42.7$ & 23.5 & 49.7 & Field & $\begin{array}{c}\text { marathon-race running, } \\
\text { TA: } 19.1^{\circ} \mathrm{C}\end{array}$ & $\begin{array}{c}6 \% \text { CHO-E beverage (Gatorade } \\
\text { Sports Science Institute, Barrington, } \\
\text { IL, USA), } 650 \mathrm{~mL} \sim 30 \mathrm{~min} \text { before the } \\
\text { start of the race and } 1000 \mathrm{~mL} \text { every } \\
60 \mathrm{~min} \text { thereafter }\end{array}$ & $0.04[-0.36,0.43]$ \\
\hline Van Essen et al., 2006 [138] & CS & $10 / \mathrm{M}$ & 24.0 & 22.9 & $63.0 *$ & $\mathrm{Lab}$ & $\begin{array}{l}80 \mathrm{~km} \text { cycling TT, } \\
\text { TA: } 20.0-23.0^{\circ} \mathrm{C}\end{array}$ & $\begin{array}{c}\text { 6\% CHO-E beverage (SUC), } 250 \mathrm{~mL} \\
\text { every } 15 \mathrm{~min}\end{array}$ & $0.19[-0.69,1.07]$ \\
\hline Watson et al., 2012 i [140] & CS & $12 / \mathrm{M}$ & 22.0 & 22.4 & 54.4 & $\mathrm{Lab}$ & $\begin{array}{c}\text { cycling to volitional exhaustion } \\
\text { at } 70 \% \dot{\mathrm{VO}}_{2} \mathrm{max}, \mathrm{TA}: 10{ }^{\circ} \mathrm{C}\end{array}$ & $\begin{array}{l}\text { 2\% CHO-E solutions (SUC:GL:FRU } \\
\text { in a ratio of 50:25:25: Tesco, Ltd., } \\
\text { Cheshunt, UK) immediately prior to } \\
\text { exercise and every } 10 \text { min during } \\
\text { exercise, served at } 21.0^{\circ} \mathrm{C}\end{array}$ & $0.19[-0.61,0.99]$ \\
\hline Watson et al., 2012 ii [140] & CS & $12 / \mathrm{M}$ & 22.0 & 22.4 & 54.4 & $\mathrm{Lab}$ & $\begin{array}{c}\text { cycling to volitional exhaustion } \\
\text { at } 70 \% \dot{\mathrm{VO}}_{2} \mathrm{max}, \mathrm{TA}: 10{ }^{\circ} \mathrm{C}\end{array}$ & $\begin{array}{l}4 \% \text { CHO-E solutions (SUC:GL:FRU } \\
\text { in a ratio of 50:25:25: Tesco, Ltd., } \\
\text { Cheshunt, UK) immediately prior to } \\
\text { exercise and every } 10 \text { min during } \\
\text { exercise, served at } 21.0^{\circ} \mathrm{C}\end{array}$ & $0.59[-0.23,1.41]$ \\
\hline Watson et al., 2012 iii [140] & CS & $12 / \mathrm{M}$ & 22.0 & 22.4 & 54.4 & $\mathrm{Lab}$ & $\begin{array}{l}\text { cycling to volitional exhaustion } \\
\text { at } 70 \% \dot{\mathrm{VO}}_{2} \max , \mathrm{TA}: 10{ }^{\circ} \mathrm{C}\end{array}$ & $\begin{array}{l}\text { 6\% CHO-E solutions (SUC:GL:FRU } \\
\text { in a ratio of 50:25:25: Tesco, Ltd., } \\
\text { Cheshunt, UK) immediately prior to } \\
\text { exercise and every } 10 \text { min during } \\
\text { exercise, served at } 21.0^{\circ} \mathrm{C}\end{array}$ & $0.60[-0.22,1.42]$ \\
\hline Watson et al., 2012 iv [140] & CS & $12 / \mathrm{M}$ & 21.0 & 25.0 & 52.4 & Lab & $\begin{array}{l}\text { cycling to volitional exhaustion } \\
\text { at } 60 \% \dot{\mathrm{VO}}_{2} \max , \mathrm{TA}: 30{ }^{\circ} \mathrm{C}\end{array}$ & $\begin{array}{l}2 \% \text { CHO-E solutions (SUC:GL:FRU } \\
\text { in a ratio of 50:25:25: Tesco, Ltd., } \\
\text { Cheshunt, UK) immediately prior to } \\
\text { exercise and every } 10 \text { min during } \\
\text { exercise, served at } 21.0^{\circ} \mathrm{C}\end{array}$ & $0.41[-0.40,1.22]$ \\
\hline
\end{tabular}


Table 1. Cont.

\begin{tabular}{|c|c|c|c|c|c|c|c|c|c|}
\hline Study/Trial † & Study Design & $\begin{array}{c}N / \\
\text { Gender }\end{array}$ & $\begin{array}{l}\text { Age } \\
\text { (year) }\end{array}$ & $\begin{array}{c}\text { BMI } \\
\left(\mathbf{k g} \cdot \mathrm{m}^{-2}\right)\end{array}$ & $\begin{array}{c}\dot{\mathrm{VO}} \mathrm{O}_{2} \max \\
\left(\mathrm{mL} \cdot \mathrm{kg}^{-1} \cdot \mathrm{min}^{-1}\right)\end{array}$ & $\begin{array}{l}\text { Lab/ } \\
\text { Field }\end{array}$ & $\begin{array}{c}\text { Exercise Task } \\
\text { (Brief Description) }\end{array}$ & $\begin{array}{l}\text { CHO Supplementation } \\
\text { (Brief Description) }\end{array}$ & $\begin{array}{l}\text { SMD IV, Random } \\
{[95 \% \text { CI) }}\end{array}$ \\
\hline Watson et al., 2012 v [140] & CS & $12 / \mathrm{M}$ & 21.0 & 25.0 & 52.4 & $\mathrm{Lab}$ & $\begin{array}{l}\text { cycling to volitional exhaustion } \\
\text { at } 60 \% \mathrm{VO}_{2} \max , \mathrm{TA}: 30{ }^{\circ} \mathrm{C}\end{array}$ & $\begin{array}{l}\text { 4\% CHO-E solutions } \\
\text { (SUC:GL:FRU in a ratio of } \\
\text { 50:25:25: Tesco, Ltd., Cheshunt, } \\
\text { UK) immediately prior to } \\
\text { exercise and every } 10 \text { min during } \\
\text { exercise, served at } 21.0^{\circ} \mathrm{C}\end{array}$ & $0.41[-0.40,1.22]$ \\
\hline Watson et al., 2012 vi [140] & CS & $12 / \mathrm{M}$ & 21.0 & 25.0 & 52.4 & $\mathrm{Lab}$ & $\begin{array}{l}\text { cycling to volitional exhaustion } \\
\text { at } 60 \% \dot{\mathrm{VO}}_{2} \max , \mathrm{TA}: 30{ }^{\circ} \mathrm{C}\end{array}$ & $\begin{array}{l}\text { 6\% CHO-E solutions } \\
\text { (SUC:GL:FRU in a ratio of } \\
\text { 50:25:25: Tesco, Ltd., Cheshunt, } \\
\text { UK) immediately prior to } \\
\text { exercise and every } 10 \text { min during } \\
\text { exercise, served at } 21.0^{\circ} \mathrm{C}\end{array}$ & $0.63[-0.19,1.46]$ \\
\hline Wilber et al., 1992 [141] & CS & $10 / \mathrm{M}$ & 30.0 & 20.9 & 64.9 & $\mathrm{Lab}$ & $\begin{array}{c}\text { TE running at } 80 \% \dot{\mathrm{VO}}_{2} \max \\
\text { TA: } 22.0{ }^{\circ} \mathrm{C}\end{array}$ & $\begin{array}{c}7 \% \text { CHO solution }(85 \% \\
\text { GL-polymer }+15 \% \text { SUC: Exceed, } \\
\text { Ross Laboratories, Columbus, } \\
\text { OH), } 5 \text { min prior to exercise } \\
(250 \mathrm{~mL}) \text { and at } 15 \text {-min intervals } \\
\text { during exercise }(150 \mathrm{~mL}) \text {, served } \\
\text { at } 5.0^{\circ} \mathrm{C}\end{array}$ & $0.85[-0.08,1.77]$ \\
\hline Williams et al., 1990 ii [142] & CS & $12 / \mathrm{M}$ & 30.8 & 21.6 & 63.4 & $\mathrm{Lab}$ & $\begin{array}{l}30 \mathrm{~km} \text {-race running, } \\
\text { TA: } 20.2{ }^{\circ} \mathrm{C}\end{array}$ & $\begin{array}{c}5 \% \text { CHO-E solution ( } 2 \% \\
\text { GL-polymer + 2\% FRU + 1\% } \\
\text { other CHO), } 5 \text { min prior to } \\
\text { exercise } 250 \mathrm{~mL} \text { and every } 5 \mathrm{~km} \\
150 \mathrm{~mL}\end{array}$ & $0.18[-0.68,1.05]$ \\
\hline
\end{tabular}

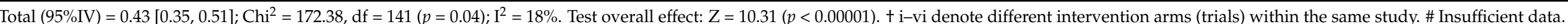

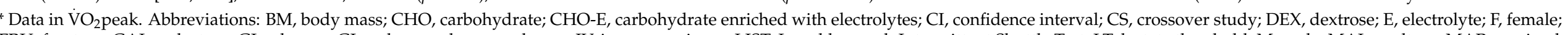

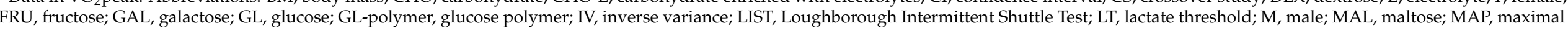

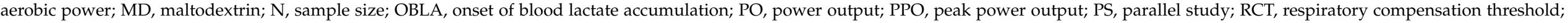

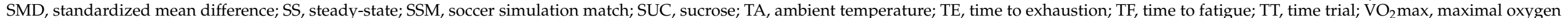
uptake; $\dot{\mathrm{VO}}_{2}$ peak, peak oxygen uptake; Vpeak, peak running velocity; VT, ventilatory threshold; WAT, Wingate anaerobic power test; Wmax, maximal power output. 


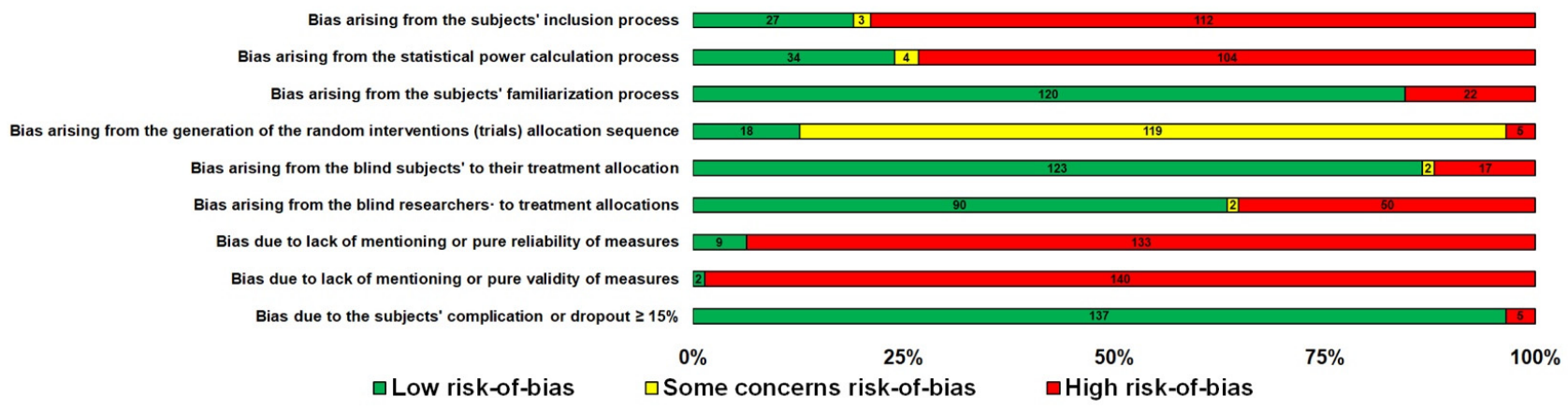

Figure 2. Overview of the authors' judgments for each risk-of-bias item as a percentage in 142 trials.

\subsection{Effect of Carbohydrate Supplementation}

Subgroup analysis showed that there were no significant differences between the age classes and subjects' gender in the effect measurement (Figures S2 and S3). However, it is worth mentioning that although there is a tendency of the effect size to be reduced as the subjects' CRF level increases according to the random-effects analysis (Figure 3), the fixed-effects analysis shows a significant effect size reduction as the subjects' CRF level increases ( $p=0.03$, Figure 54 ).

Regarding the exercise task, subgroup analysis showed that there were no significant differences between exercise modes, protocol tests, or type classes in the effect measurement (Figures S5-S7). What is remarkable, however, is the greater SMD in the cycling $(0.47[0.38,0.57])$ compared to running mode $(0.35[0.17,0.52])$ subgroup classes, though the effect is not significantly different. The effect of $\mathrm{CHO}$ interventions compared to control trials in the four exercise task duration time classes was significantly different between classes, $(p \leq 0.05$, Figure 4$)$. This revealed the advantageous role of $\mathrm{CHO}$ supplementation when $\mathrm{CHO}$ supplements are ingested during endurance exercise lasting $1-2 \mathrm{~h}(0.41[0.27,0.55])$ or $2-4 \mathrm{~h}(0.51[0.40,0.62])$ in comparison to exercise sessions lasting less than $1 \mathrm{~h}(0.15[-0.13,0.43])$ or more than $4 \mathrm{~h}(0.19[-0.16,0.55])$. 
Std. Mean Difference Weight IV, Random, $95 \% \mathrm{Cl}$

Std. Mean Difference

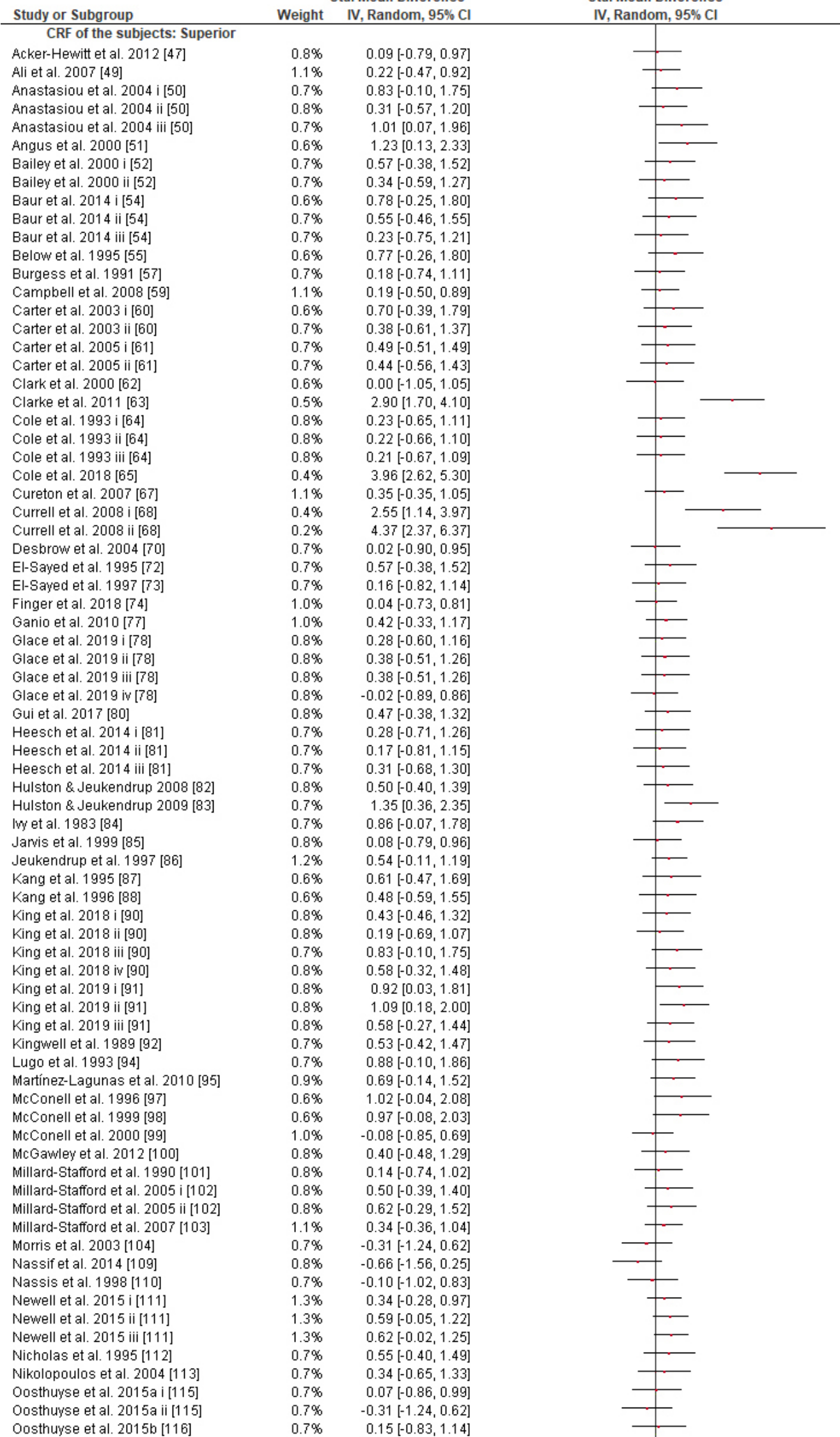

Figure 3. Cont. 


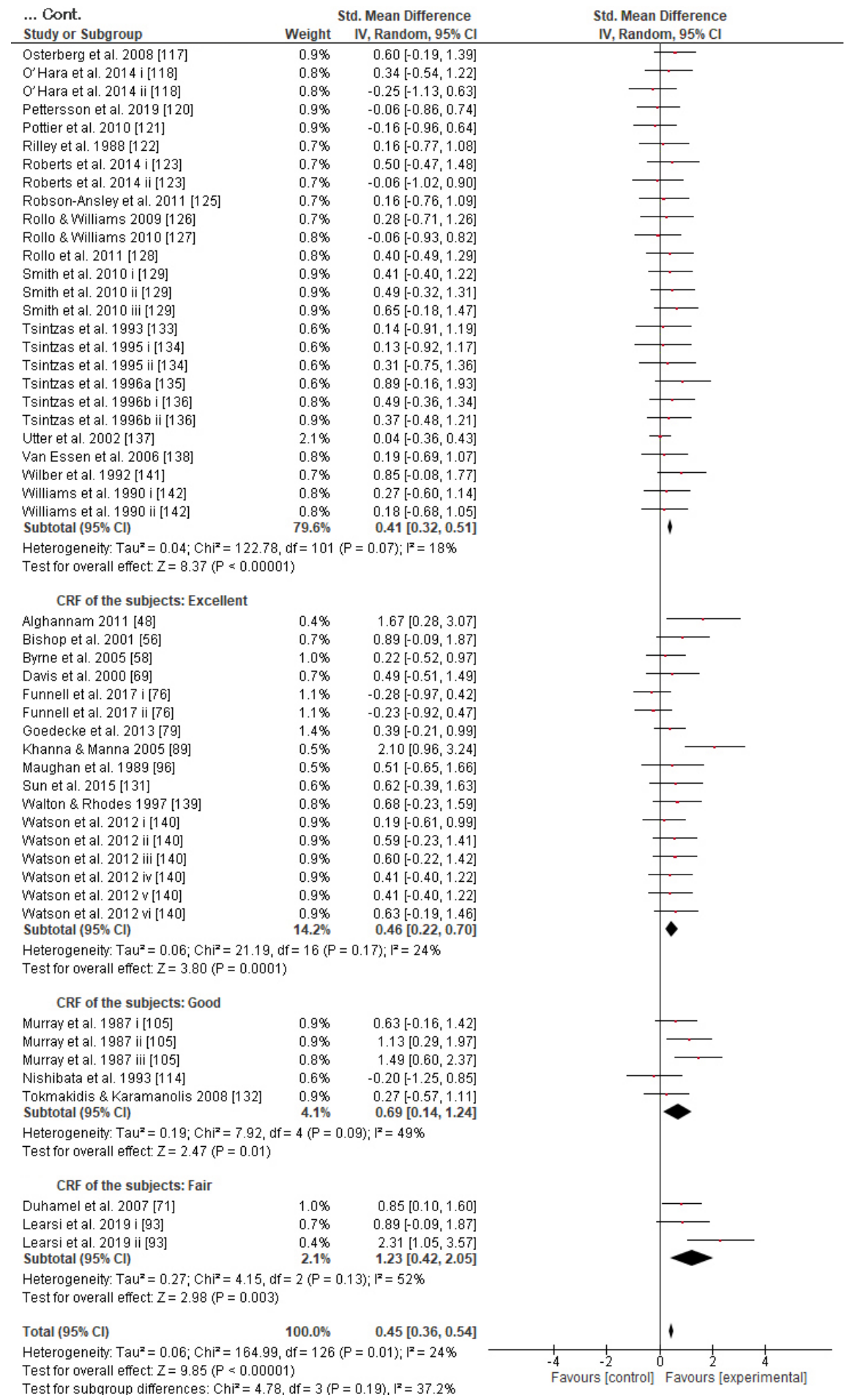

Figure 3. Forest plot shows the effects (red square symbol) of experimental carbohydrate supplementation as compared to a control on exercise outcome for 127 interventions [47-52,54-65,67-74,76-105,109-118,120-123,125-129,131,133-142]. Subgroup analyses show the results with regards to subjects' characteristics in four CRF groups (superior, excellent, good, and fair). The black diamond symbol at the subgroups and at the bottom of the figure represents the standardized mean difference with the $95 \%$ confidence intervals for all interventions following random effects meta-analyses. Studies or trials that provided insufficient data for subgroup classification were not included. Abbreviations: CI, confidence interval; CRF, cardio-respiratory fitness; IV, inverse variance; SD, standard deviation; Std, standardized; i-vi denote different intervention arms (trials) within the same study. 
Std. Mean Difference

\begin{tabular}{|c|c|c|}
\hline Study or Subgroup & Weight & IV, Random, $95 \% \mathrm{C}$ \\
\hline \multicolumn{3}{|c|}{$45 \mathrm{~min} \leq$ Exercise duration time $\leq 60 \mathrm{~min}$} \\
\hline Below et al. 1995 [55] & $0.5 \%$ & $0.77[-0.26,1.80]$ \\
\hline Carter et al. 2003 ii [60] & $0.6 \%$ & $0.38[-0.61,1.37]$ \\
\hline Clark et al. 2000 [62] & $0.5 \%$ & $0.00[-1.05,1.05]$ \\
\hline El-Sayed et al. 1997 [73] & $0.6 \%$ & $0.16[-0.82,1.1$ \\
\hline Funnell et al. $2017 \mathrm{i}[76]$ & $1.0 \%$ & $-0.28[-0.97,0.42]-2]$ \\
\hline Jarvis et al. 1999 [85] & $0.7 \%$ & $0.08[-0.79,0.9$ \\
\hline Nikolopoulos et al. 2004 [113] & $0.6 \%$ & $0.34[-0.65,1.3$ \\
\hline Rollo \& Williams 2009 [126] & $0.6 \%$ & $0.28[-0.71,1.2$ \\
\hline Rollo \& Williams 2010 [127] & $0.7 \%$ & $-0.06[-0.93,0.8$ \\
\hline Rollo et al. 2011 [128] & $0.7 \%$ & $0.40[-0.49,1.2$ \\
\hline Steiner et al. 2009 [130] & $0.7 \%$ & $0.07[-0.85,1$ \\
\hline Subtotal $(95 \% \mathrm{Cl})$ & $7.2 \%$ & $0.15[-0.13,0$. \\
\hline
\end{tabular}

Heterogeneity: $\operatorname{Tau}^{2}=0.00 ; \mathrm{Chi}^{2}=3.89, \mathrm{df}=10(\mathrm{P}=0.95) ; \mathrm{I}^{2}=0 \%$

Test for overall effect: $Z=1.07(P=0.28)$

$60 \mathrm{~min}<$ Exercise duration time $\leq 120 \mathrm{~min}$ Acker-Hewitt et al. $2012[47] \quad 0.7 \%$ Acker-Hewitt et al. 2012 [47] $\quad 0.7 \%$

$2011[4$

Ali et al. 2007 [49]

Anastasiou et al. $2004 \mathrm{i}[50]$

Anastasiou et al. 2004 ii [50]

Anastasiou et al. 2004 iii [50]

Baldassarre et al. 2021 [53]

Bishop et al. 2001 [56]

Campbell et al. 2008 [59]

Clarke et al. 2011 [63]

Cole et al. 1993 i [64]

Cole et al. 1993 ii [64]

Cole et al. 1993 iii [64]

Cole et al. 2018 [65]

Coletta et al. $2013 \mathrm{i}[66]$

Coletta et al. 2013 ii [66]

Davis et al. 2000 [69]

Desbrow et al. $2004[70]$

Duhamel et al. 2007 [71]

El-Sayed et al. 1995 [72]

Finger et al. 2018 [74]

Fulco et al. 2007 i [75]

Fulco et al. 2007 ii [75]

Funnell et al. $2017 \mathrm{ii}$ [76]

Goedecke et al. 2013 [79]

Jeukendrup et al. 1997 [86]

Khanna \& Manna 2005 [89]

Maughan et al. 1989 [96]

Mcconell et al. 2000 [99]

Morris et al. 2003 [104]

Murray et al. $1989 \mathrm{i}[106]$

Murray et al. $1989 \mathrm{ii}[106]$

Murray et al. 1989 iii [106]

Naclerio et al. 2014 [108]

Nassis et al. 1998 [110]

Nicholas et al. 1995 [112]

Nishibata et al 1993 [114]

O'Hara et al. 2014 i [118]

$\mathrm{O}^{\prime}$ Hara et al. $2014 \mathrm{ii}$ [118]

O'Neal et al. 2013 [119]

Pettersson et al. 2019 [120]

Pottier et al. 2010 [121]

Rilley et al. 1988 [122]

Robson-Ansley et al. 2009 [124]

Sun et al. 2015 [131]

Tokmakidis \& Karamanolis 2008 [132]

Tsintzas et al. 1996a [135]

Tsintzas et al. 1996b i [136]

Tsintzas et al. 1996b ii [136]

Walton \& Rhodes 199? [139]

Watson et al. 2012 i [1 40]

Watson et al. 2012 ii [140]

Watson et al. 2012 iii [140]

Watson et al. $2012 \mathrm{iv}[140]$

Watson et al. $2012 v[140]$

Watson et al. 2012 vi [140]

Wilber et al. 1992 [141]

Subtotal $(95 \% \mathrm{Cl})$

$0.3 \%$
$1.0 \%$

$1.0 \%$
$0.7 \%$

$0.7 \%$

$0.5 \%$

$0.6 \%$
$1.0 \%$
$0.4 \%$

$0.4 \%$
$0.7 \%$
$0.7 \%$

$0.7 \%$

$0.3 \%$

$0.8 \%$

$0.8 \%$

$0.6 \%$

$0.7 \%$

$0.9 \%$

$0.6 \%$

$0.6 \%$

$0.9 \%$

$0.5 \%$

$1.0 \%$

$1.3 \%$

$1.1 \%$

$0.5 \%$

$0.4 \%$

$0.4 \%$

$0.9 \%$

$0.6 \%$

$0.8 \%$

$0.8 \%$

$0.8 \%$

$0.7 \%$

$0.7 \%$

$0.7 \%$

$0.5 \%$

$0.7 \%$

$0.7 \%$

$1.7 \%$

$0.8 \%$

$8 \%$

$0.8 \%$

$0.5 \%$

$0.8 \%$

$0.5 \%$

$0.7 \%$

$0.8 \%$

$0.8 \%$

$.7 \%$

$0.8 \%$
$0.8 \%$
$0.8 \%$

$0.8 \%$

$0.8 \%$

$0.8 \%$

$0.7 \%$

$0.09[-0.79,0.97]$

$1.67[0.28,3.07]$

$.22[-0.47,0.92]$

$0.83[-0.10,1.75]$

$0.31[-0.57,1.20]$

$1.01[0.07,1.96]$

$.83[-0.21,1.86]$

$0.89[-0.09,1.87]$

$0.19[-0.50,0.89]$

$2.90[1.70,4.10]$

$0.23[-0.65,1.11]$

$0.22[-0.66,1.10]$

$0.21[-0.67,1.09]$

$3.96[2.62,5.30]$

$0.08[-0.72,0.88]$

$-0.07[-0.87,0.73]$

$0.49[-0.51,1.49]$

$0.02[-0.90,0.95]$

$0.85[0.10,1.60]$

$0.57[-0.38,1.52]$

$0.04[-0.73,0.81]$

$0.58[-0.48,1.64]$

$0.43[-0.62,1.48]$

$-0.23[-0.92,0.47]$

$0.39[-0.21,0.99]$

$0.54[-0.11,1.19]$

$2.10[0.96,3.24]$

$0.51[-0.65,1.66]$

$0.51[-0.65,1.66]$

$-0.31[-1.24,0.62]$

$0.57[-0.25,1.39]$

$0.30[-0.50,1.11]$

$0.05[-0.75,0.85]$

$-0.01[-0.89,0.87$

$-0.10[-1.02,0.83]$

$0.55[-0.40,1.49]$

$-0.20[-1.25,0.85]$

$0.34[-0.54,1.22]$

$-0.25[-1.13,0.63]$

$0.10[-0.36,0.56]$

$-0.06[-0.86,0.74]$

$-0.16[-0.96,0.64]$

$0.16[-0.77,1.08]$

$0.44[-0.63,1.50]$

$0.62[-0.39,1.63]$

$0.27[-0.57,1.11]$

$0.89[-0.16,1.93]$

$0.49[-0.36,1.34]$

$0.37[-0.48,1.21]$

$0.68[-0.23,1.59]$

$0.19[-0.61,0.99]$

$0.59[-0.23,1.41]$

$0.60[-0.22,1.42]$

$0.41[-0.40,1.22]$

$0.41[-0.40,1.22]$

$0.63[-0.19 .1 .46]$

$0.85[-0.08,1.77]$

Test for overall effect: $Z=5.60$ ( $P<0.00001)$

$120 \mathrm{~min}<$ Exercise duration time $\leq 240 \mathrm{~min}$

Angus et al. 2000 [51]

Bailey et al. 2000 i [52]

Bailey et al. 2000 ii [52]

Baur et al. 2014 i [54]

Baur et al. 2014 ii [54]

$0.5 \%$
$0.6 \%$

$0.6 \%$

$0.6 \%$
$0.5 \%$
$0.6 \%$

$0.6 \%$

$23[0.13,2.33]$

$0.57[-0.38,1.52]$

$0.34[-0.59,1.27]$

$0.78[-0.25,1.80]$

$0.55[-0.46,1.55]$

$0.23[-0.75,1.21]$
Std. Mean Difference

IV. Random $95 \% \mathrm{Cl}$

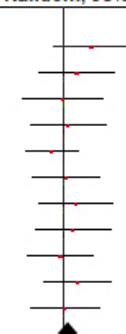

Figure 4. Cont. 


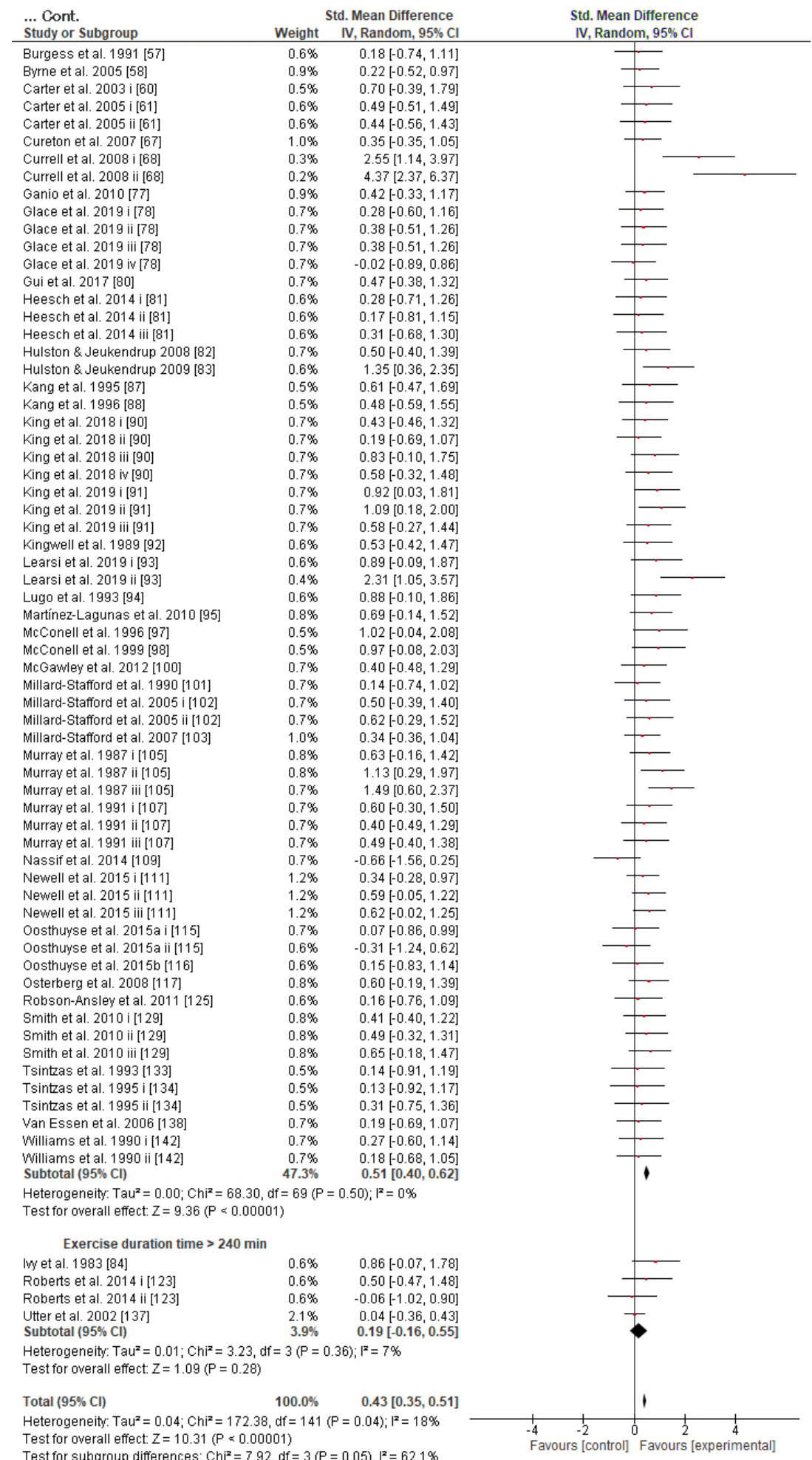

Figure 4. Forest plot shows the effects (red square symbol) of experimental carbohydrate supplementation as compared to a control on exercise outcome for 142 interventions [47-142]. Subgroup analyses show the results with regards to the exercise task in four exercise duration time groups (45 $\min \leq \mathrm{T} \leq 60 \mathrm{~min}, 60 \mathrm{~min}<\mathrm{T} \leq 120 \mathrm{~min}, 120 \mathrm{~min}<\mathrm{T} \leq 240 \mathrm{~min}, \mathrm{~T}>240 \mathrm{~min}$ ). The black diamond symbol at the subgroups and at the bottom of the figure represents the standardized mean difference with the $95 \%$ confidence intervals for all interventions following random effects meta-analyses. Studies or trials that provided insufficient data for subgroup classification were not included. Abbreviations: CI, confidence interval; IV, inverse variance; SD, standard deviation; Std, standardized; T, time; i-vi denote different intervention arms (trials) within the same study. 
Concerning the $\mathrm{CHO}$ supplementation, subgroup analysis showed that there were no significant differences in the SMD between different $\mathrm{CHO}$ supplementation concentrations, doses, types, composites, formulations, administration times, and temperatures of supplement administration classes (Figures S8-S13). It was, however, found by the random-effects analysis that $\mathrm{CHO}$ supplementation composed of GL:FRU has a slight tendency for a greater effect on performance in comparison to other MTC compositions (marginally insignificant, Figure 5). This tendency becomes a significantly greater effect of GL:FRU formation on performance in comparison to other MTC compositions using the fixed-effects analysis model, ( $p=0.04$, Figure S14). Further subgroup analysis showed that the effect measurement of the $\mathrm{CHO}$ supplement ingested was significantly higher when a double-source $\mathrm{CHO}$ solution formulation $(0.57[0.37,0.76])$ was used, in comparison to a triple-or-more-source $\mathrm{CHO}$ solution formulation $(0.30$ [0.11, 0.49]), $(p \leq 0.05$; Figure 6). Regarding the administration of high $\mathrm{CHO}$ doses, the effect of $\mathrm{CHO}$ dose at rates $>100 \mathrm{~g} \cdot \mathrm{h}^{-1}$ had a significantly lower effect measurement $(0.17[-0.23,0.57])$ in comparison to dose rates of $81-100 \mathrm{~g} \cdot \mathrm{h}^{-1}(0.82[0.31,1.34]),(p \leq 0.05$, Figure 7$)$. Moreover, CHO supplementation during exercise had a significantly higher effect $(0.47[0.37,0.58])$ in comparison to $\mathrm{CHO}$ supplementation when administered prior to or at the beginning of the exercise $(0.12[-0.21,0.44])(p \leq 0.05$, Figure 8$)$. Lastly, not enough evidence was found to confirm or reject the hypothesis that the absence of differences in the effect measurement between different ambient thermal conditions after $\mathrm{CHO}$ supplementation was due to the limited studies available (Figure S15).

\begin{tabular}{|c|c|c|c|}
\hline \multirow{2}{*}{$\frac{\text { Study or Subgroup }}{\text { GL:FRU solution }}$} & \multicolumn{2}{|c|}{ Std. Mean Difference } & Std. Mean Difference \\
\hline & & & \\
\hline Baldassarre et al. 2021 [53] & $1.5 \%$ & $0.83[-0.21,1.86]$ & \\
\hline Baur et al. $2014 \mathrm{i}$ [54] & $1.6 \%$ & $0.78[-0.25,1.80]$ & \\
\hline Cole et al. 1993 iii [64] & $2.1 \%$ & $0.21[-0.67,1.09]$ & — \\
\hline Currell et al. $2008 \mathrm{ii} \mathrm{[68]}$ & $0.4 \%$ & $4.37[2.37,6.37]$ & \\
\hline Hulston \& Jeukendrup 2009 [83] & $1.7 \%$ & $1.35[0.36,2.35]$ & $\longrightarrow$ \\
\hline King et al. 2018 iii [90] & $1.9 \%$ & $0.83[-0.10,1.75]$ & \\
\hline King et al. $2018 \mathrm{iv}[90]$ & $2.0 \%$ & $0.58[-0.32,1.48]$ & - \\
\hline King et al. $2019 \mathrm{i}$ [91] & $2.0 \%$ & $0.92[0.03,1.81]$ & \\
\hline King et al. $2019 \mathrm{ii}[91]$ & $2.0 \%$ & $1.09[0.18,2.00]$ & 一 \\
\hline King et al. 2019 iii [91] & $2.2 \%$ & $0.58[-0.27,1.44]$ & - \\
\hline Millard-Stafford et al. 1990 [101] & $2.1 \%$ & $0.14[-0.74,1.02]$ & - \\
\hline Murray et al. $1987 \mathrm{iii}[105]$ & $2.0 \%$ & $1.49[0.60,2.37]$ & - \\
\hline Rilley et al. 1988 [122] & $1.9 \%$ & $0.16[-0.77,1.08]$ & - \\
\hline Subtotal $(95 \% \mathrm{Cl})$ & $23.3 \%$ & $0.84[0.48,1.20]$ & $\diamond$ \\
\hline \multicolumn{3}{|c|}{ Heterogeneity: $\operatorname{Tau}^{2}=0.21 ; \mathrm{Chi}^{2}=22.45, \mathrm{df}=12(\mathrm{P}=0.03) ; \mathrm{I}^{2}=47 \%$} & $\begin{array}{l}\text { Heterogeneity: } \operatorname{Tau}^{2}=0.21 ; \mathrm{Chi}^{2}=22.45, \mathrm{df}=12(\mathrm{P}=0.03) ; \mathrm{I}^{2}=47 \% \\
\text { Test for overall effect: } Z=4.51(P<0.00001)\end{array}$ \\
\hline \multicolumn{4}{|l|}{ GL:SUC solution } \\
\hline Cole et al. $1993 \mathrm{i}[64]$ & $2.1 \%$ & $0.23[-0.65,1.11]$ & - \\
\hline Kang et al. $1995[87]$ & $1.4 \%$ & $0.61[-0.47,1.69]$ & \\
\hline Kang et al. $1996[88]$ & $1.4 \%$ & $0.48[-0.59,1.55]$ & 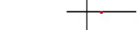 \\
\hline Millard-Stafford et al. 2005 i [102] & $2.0 \%$ & $0.50[-0.39,1.40]$ & 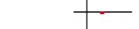 \\
\hline Murray et al. 1987 ii [105] & $2.3 \%$ & $1.13[0.29,1.97]$ & . \\
\hline Pottier et al. 2010 [121] & $2.4 \%$ & $-0.16[-0.96,0.64]$ & - \\
\hline Wilber et al. 1992 [141] & $1.9 \%$ & $0.85[-0.08,1.77]$ & \\
\hline Subtotal $(95 \% \mathrm{Cl})$ & $13.5 \%$ & $0.50[0.16,0.85]$ & $\bullet$ \\
\hline \multirow{2}{*}{\multicolumn{4}{|c|}{$\begin{array}{l}\text { Heterogeneity: } \text { Tau }^{2}=0.00 ; \mathrm{Ch}^{2}=5.72, \mathrm{df}=6(\mathrm{P}=0.46) ; \mathrm{I}^{2}=0 \% \\
\text { Test for overall effect: } Z=2.86(\mathrm{P}=0.004)\end{array}$}} \\
\hline & & & \\
\hline \multicolumn{4}{|l|}{ GL:MD solution } \\
\hline Javis et al. 1999 [85] & $2.1 \%$ & $0.08[-0.79,0.96]$ & \\
\hline Subtotal $(95 \% \mathrm{Cl})$ & $2.1 \%$ & $0.08[-0.79,0.96]$ & \\
\hline \multicolumn{4}{|l|}{ Heterogeneity: Not applicable } \\
\hline \multicolumn{4}{|c|}{ Test for overall effect: $Z=0.19(P=0.85)$} \\
\hline \multicolumn{4}{|l|}{ MD:FRU solution } \\
\hline McGawley et al. 2012 [100] & $2.0 \%$ & $0.40[-0.48,1.29]$ & \\
\hline Oosthuyse et al. 2015a i [115] & $1.9 \%$ & $0.07[-0.86,0.99]$ & \\
\hline Oosthuyse et al. 2015b [116] & $1.7 \%$ & $0.15[-0.83,1.14]$ & \\
\hline Pettersson et al. 2019 [120] & $2.5 \%$ & $-0.06[-0.86,0.74]$ & \\
\hline Roberts et al. 2014 i [123] & $1.7 \%$ & $0.50[-0.47,1.48]$ & \\
\hline Subtotal $(95 \% \mathrm{Cl})$ & $9.8 \%$ & $0.20[-0.21,0.60]$ & \\
\hline \multicolumn{4}{|c|}{$\begin{array}{l}\text { Heterogeneity: Tau }{ }^{2}=0.00 ; \mathrm{Chi}^{2}=1.07, \mathrm{df}=4(\mathrm{P}=0.90) ; \mathrm{I}^{2}=0 \% \\
\text { Test for overall effect: } Z=0.95(P=0.34)\end{array}$} \\
\hline \multicolumn{4}{|l|}{ MD:DEX solution } \\
\hline Murray et al. 1991 ii [107] & $2.0 \%$ & $0.40[-0.49,1.29]$ & \\
\hline Murray et al. $1991 \mathrm{iii} \mathrm{[107]}$ & $2.0 \%$ & $0.49[-0.40,1.38]$ & \\
\hline Subtotal $(95 \% \mathrm{Cl})$ & $4.1 \%$ & $0.45[-0.18,1.08]$ & \\
\hline \multirow{2}{*}{\multicolumn{4}{|c|}{$\begin{array}{l}\text { Heterogeneity: } \operatorname{Tau}^{2}=0.00 ; C h \mathrm{i}^{2}=0.02, \mathrm{df}=1(\mathrm{P}=0.89) ; \mathrm{I}^{2}=0 \% \\
\text { Test for overall effect: } Z=1.39(P=0.16)\end{array}$}} \\
\hline & & & \\
\hline \multicolumn{4}{|l|}{ MD:SUC solution } \\
\hline $\begin{array}{l}\text { Tsintzas et al. } 1995 \text { i [134] } \\
\text { Subtotal (95\% CI) }\end{array}$ & $\begin{array}{l}1.5 \% \\
1.5 \%\end{array}$ & $\begin{array}{l}0.13[-0.92,1.17] \\
0.13[-0.92,1.17]\end{array}$ & \\
\hline Subtotal $(95 \% \mathrm{Cl})$ & $1.5 \%$ & $0.13[-0.92,1.17]$ & \\
\hline $\begin{array}{l}\text { Heterogeneity. Not applicable } \\
\text { Test for overall effect } z=0.23\end{array}$ & & & \\
\hline
\end{tabular}

Figure 5. Cont. 


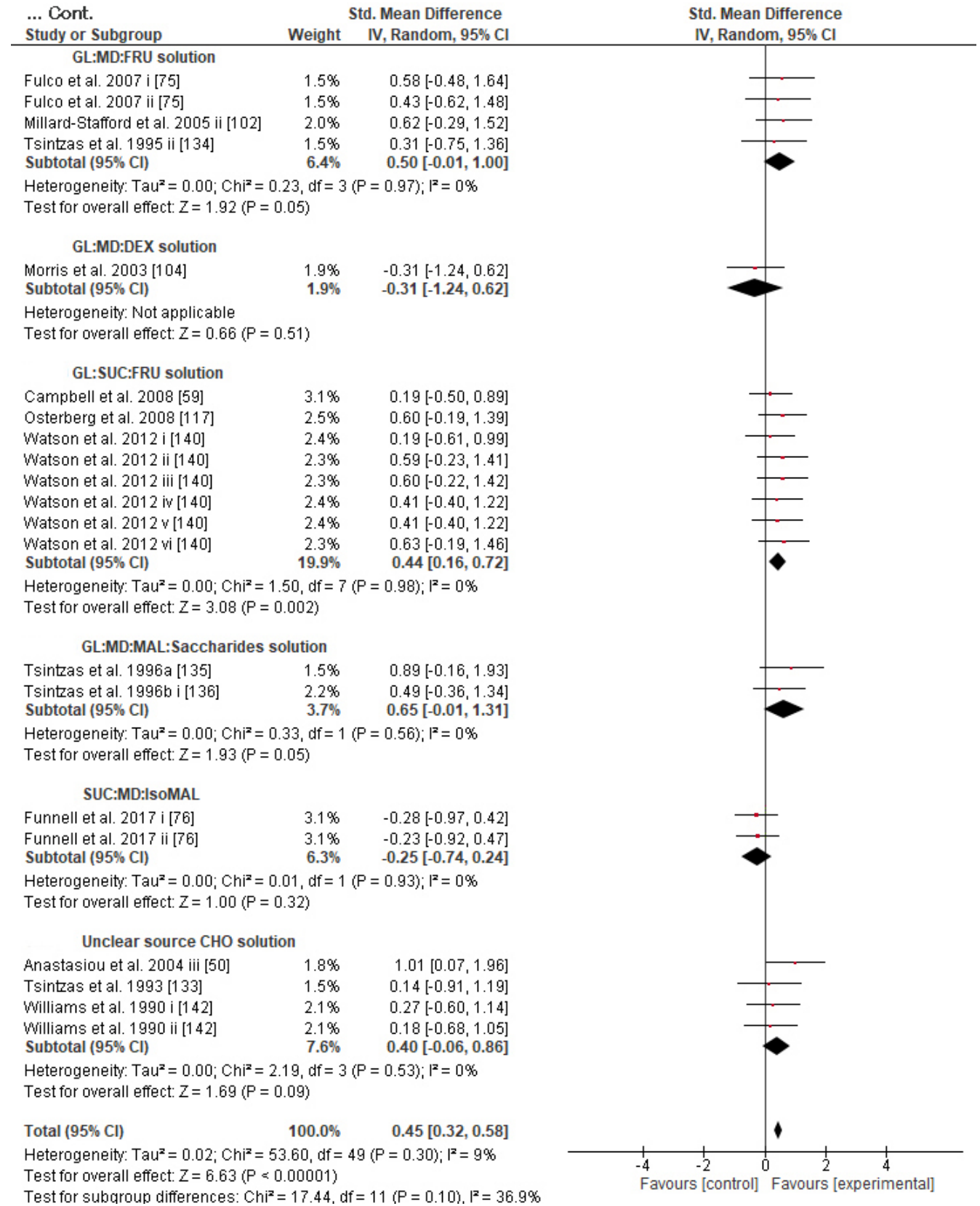

Figure 5. Forest plot shows the effects (red square symbol) of experimental carbohydrate supplementation as compared to a control on exercise outcome for 50 interventions $[50,53,54,59,64,68,75,76,83,85$, $87,88,90,91,100-102,104,105,107,117,121,122,133-136,140-142]$. Subgroup analyses show the results with regards to supplementation in 12 MTC groups (GL:FRU, GL:SUC, GL:MD, MD:FRU, MD:DEX, MD:SUC, GL:MD:FRU, GL:MD:DEX, GL:SUC:FRU, GL:MD:MAL:Saccharides, SUC:MD:IsoMAL, unclear $\mathrm{CHO}$ substances mixture). The black diamond symbol at the subgroups and at the bottom of the figure represents the standardized mean difference with the $95 \%$ confidence intervals for all interventions following random effects meta-analyses. Studies or trials that provided insufficient data for subgroup classification were not included. Abbreviations: $\mathrm{CHO}$, carbohydrate, $\mathrm{CI}$, confidence interval; DEX, dextrose; FRU, fructose; GAL, galactose; GL, glucose; IV, inverse variance; MD, maltodextrin; MAL, maltose; MTC, multiple transportable carbohydrate; SD, standard deviation; Std, standardized; SUC, sucrose; i-vi denote different intervention arms (trials) within the same study. 


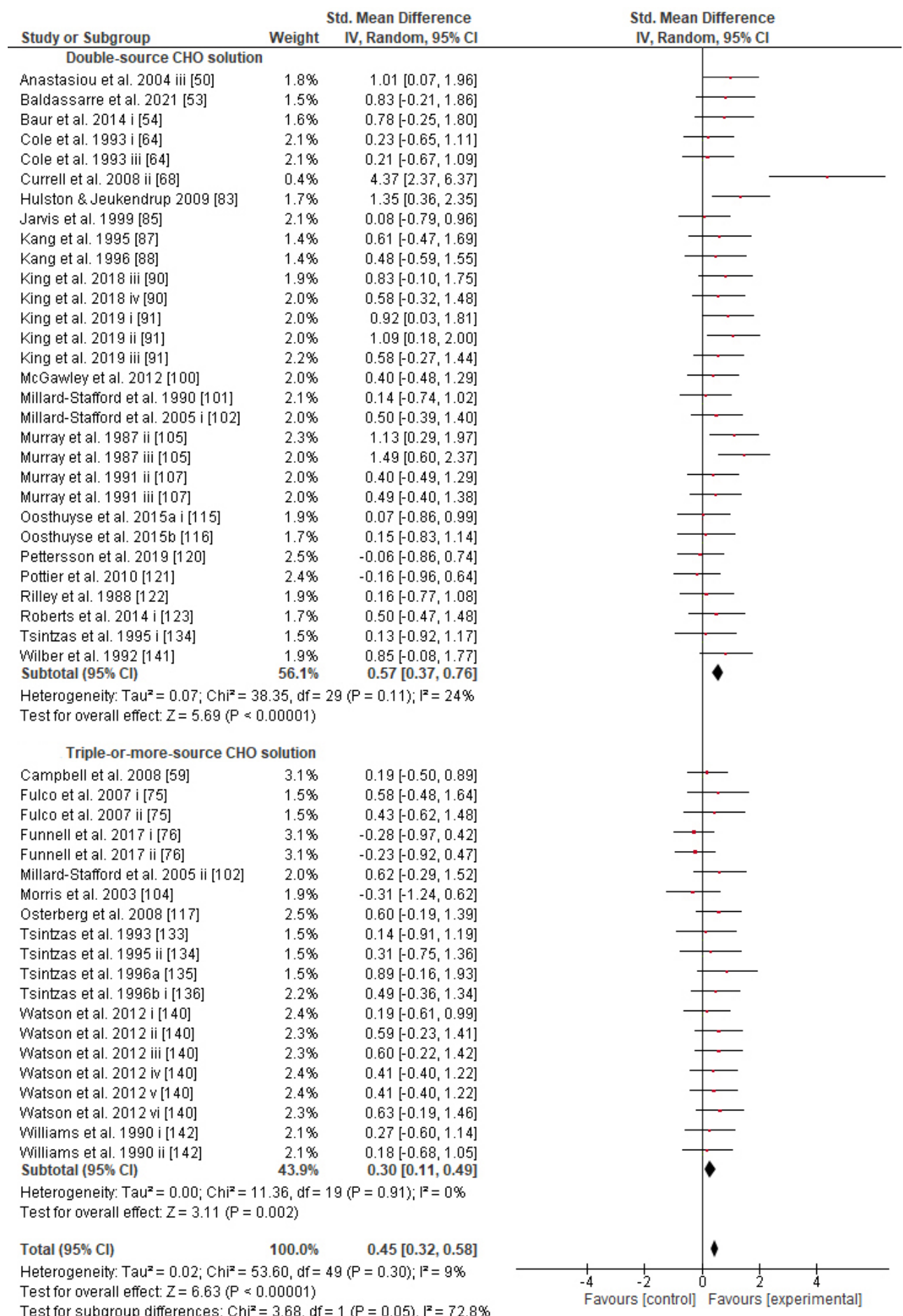

Figure 6. Forest plot shows the effects (red square symbol) of experimental carbohydrate supplementation as compared to a control on exercise outcome for 50 interventions $[50,53,54,59,64,68,75,76,83,85,87,88,90,91,100-105,107,115-117,120-123,133-136,140-142]$. Subgroup analyses show the results with regards to supplementation in two carbohydrate formulation groups (double-source $\mathrm{CHO}$ solution, triple-or-more-source $\mathrm{CHO}$ solution). The black diamond symbol at the subgroups and at the bottom of the figure represents the standardized mean difference with the $95 \%$ confidence intervals for all interventions following random effects meta-analyses. Studies or trials that provided insufficient data for subgroup classification were not included. Abbreviations: $\mathrm{CHO}$, carbohydrate; $\mathrm{CI}$, confidence interval; IV, inverse variance; SD, standard deviation; Std, standardized; i-vi denote different intervention arms (trials) within the same study. 


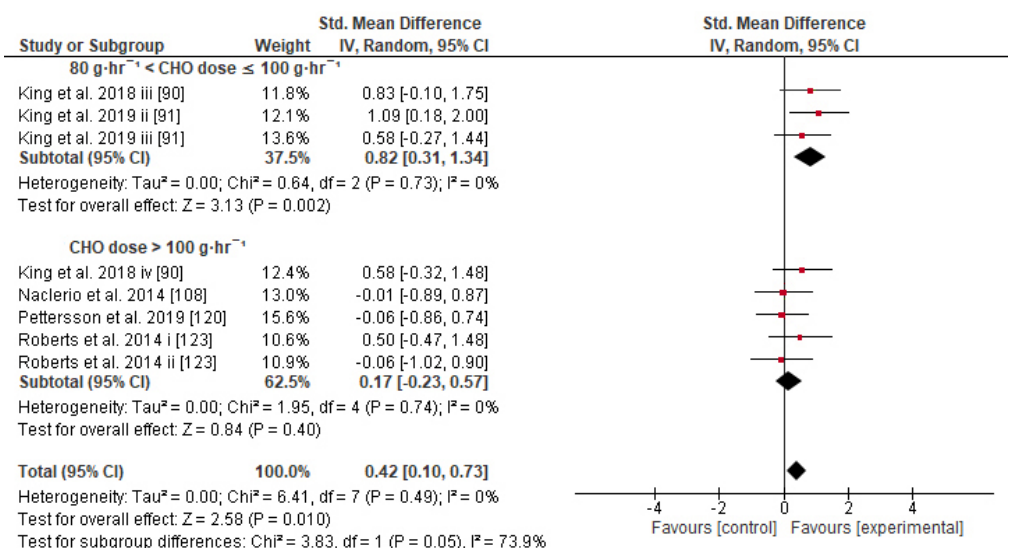

Figure 7. Forest plot shows the effects (red square symbol) of experimental carbohydrate supplementation as compared to a control on exercise outcome for 8 interventions [90,91,108,120,123]. Subgroup analyses show the results with regards to supplementation in two carbohydrate dose groups $\left(80 \mathrm{~g} \cdot \mathrm{h}^{-1}\right.$ $<\mathrm{CHO}$ dose $\leq 100 \mathrm{~g} \cdot \mathrm{h}^{-1}, \mathrm{CHO}$ dose $\left.>100 \mathrm{~g} \cdot \mathrm{h}^{-1}\right)$. The black diamond symbol at the subgroups and at the bottom of the figure represents the standardized mean difference with the $95 \%$ confidence intervals for all interventions following random effects meta-analyses. Studies or trials that provided insufficient data for subgroup classification were not included. Abbreviations: $\mathrm{CHO}$, carbohydrate; $\mathrm{CI}$, confidence interval; IV, inverse variance; SD, standard deviation; Std, standardized; i-vi denote different intervention arms (trials) within the same study.

\begin{tabular}{|c|c|c|c|}
\hline \multirow{2}{*}{\multicolumn{3}{|c|}{$\begin{array}{cc}\text { Std. Mean Difference } \\
\text { Study or Subgroup } & \text { Weight IV, Random, 95\% Cl } \\
\text { Administration time of CHO supplementation: Prior to or at the beginning of exercise }\end{array}$}} & $\begin{array}{l}\text { Std. Mean Differenc } \\
\text { IV, Random, 95\% Cl}\end{array}$ \\
\hline & & & \\
\hline & & & 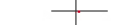 \\
\hline Funnell et al. 2017 [ [76] & $1.3 \%$ & $-0.28[-0.97,0.42]$ & \\
\hline Heesch et al. 2014 ii [81] & $0.8 \%$ & $0.17[-0.81,1.15]$ & — \\
\hline $\mathrm{O}^{\prime}$ Hara et al. $2014 \mathrm{i}[118]$ & $0.9 \%$ & $0.34-0.54,1.22]$ & \\
\hline $\mathrm{O}^{\prime}$ Hara et al. $2014 \mathrm{ii}[118]$ & $0.9 \%$ & $-0.25[-1.13,0.63]$ & - \\
\hline Tokmakidis \& Karamanolis 2008 [132] & $1.0 \%$ & $0.27[-0.57,1.11]$ & 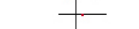 \\
\hline Walton \& Rhodes 1997 [139] & & $0.68[-0.23,1.59]$ & \\
\hline Subtotal $(95 \% \mathrm{Cl})$ & $6.5 \%$ & $0.12[-0.21,0.44]$ & $\rightarrow$ \\
\hline \multicolumn{4}{|c|}{$\begin{array}{l}\text { Heterogeneity: Tau }=0.00 ; \mathrm{Chi}^{2}=3.74 \text {, df }=6(P=0.71) ; \mathrm{l}^{2}=0 \% \\
\text { Test for overall effect: } Z=0.70(P=0.48)\end{array}$} \\
\hline \multicolumn{4}{|c|}{ Administration time of $\mathrm{CHO}$ supplementation: During exercise } \\
\hline Ali et al. $2007[49]$ & $1.3 \%$ & $0.22[-0.47,0.92]$ & — \\
\hline Anastasiou et al. 2004 i [50] & $0.9 \%$ & $0.83[-0.10,1.75]$ & - \\
\hline Anastasiou et al. 2004 ii [50] & $0.9 \%$ & $0.31[-0.57,1.20]$ & - \\
\hline Anastasiou et al. 2004 iii [50] & $0.8 \%$ & $1.01[0.07,1.96]$ & \\
\hline Angus et al. 2000 [51] & $0.6 \%$ & $1.23[0.13,2.33]$ & \\
\hline Bailey et al. 2000 i [52] & $0.8 \%$ & $0.57[-0.38,1.52]$ & - - \\
\hline Bailey et al. 2000 ii $[52]$ & $0.8 \%$ & $0.34[-0.59,1.27]$ & - \\
\hline Baldassarre et al. 2021 [53] & $0.7 \%$ & $\begin{array}{l}0.83[-0.59,1.27] \\
0.021,1.86]\end{array}$ & \\
\hline $\begin{array}{l}\text { Bad awsare et al } 1995[55] \\
\text { Below }\end{array}$ & $0.7 \%$ & $\begin{array}{l}0.87[-0.27,1,86] \\
0.77-0.26,1.80]\end{array}$ & —. \\
\hline Bishop et al. $2001[56]$ & $0.8 \%$ & $0.89[-0.09,1.87]$ & 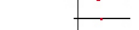 \\
\hline $\begin{array}{l}\text { Eishoppe ta. 2.2017) } \\
\text { Burges et al. } 1991 \text { [57] }\end{array}$ & $0.8 \%$ & $\begin{array}{l}0.89[-0.09,1.87] \\
0.18[-0.74,1.11]\end{array}$ & E \\
\hline Byme et al. $2005[58]$ & $1.2 \%$ & $0.22[-0.52,0.97]$ & — \\
\hline Campbell et al. 2008 [59] & $1.3 \%$ & $0.19[-0.50,0.89]$ & - \\
\hline Carter et al. $2003 \mathrm{i}[60]$ & $0.7 \%$ & $0.70[-0.39,1.79]$ & . \\
\hline Carter et al. 2003 ii $[60]$ & $0.8 \%$ & $0.38[-0.61,1.37]$ & - \\
\hline Carter et al. $2005 i[61]$ & $0.8 \%$ & $0.49[-0.51,1.49]$ & - \\
\hline Carter et al. $2005 \mathrm{ii}[61]$ & $0.8 \%$ & $0.44[-0.56,1.43]$ & - \\
\hline Clark et al. 2000 [62] & $0.7 \%$ & $0.00[-1.05,1.05]$ & - \\
\hline Clarke et al. 2011 [63] & $0.6 \%$ & $2.90[1.70,4.10]$ & \\
\hline Cole et al. 1993 i [64] & $0.9 \%$ & $0.23[-0.65,1.11]$ & — \\
\hline Cole et al. 1993 ii [64] & $0.9 \%$ & $0.22[-0.66,1.10]$ & - \\
\hline Cole et al. 1993 iii [64] & $0.9 \%$ & $0.21[-0.67,1.09]$ & - \\
\hline Cole et al. $2018[65]$ & $0.5 \%$ & $3.96[2.62,5.30]$ & \\
\hline Coletta et al. $2013 \mathrm{i}[66]$ & $1.1 \%$ & $0.08[-0.72,0.88]$ & - \\
\hline Coletta et al. 2013 ii [66] & $1.1 \%$ & $-0.07[-0.87,0.73]$ & - \\
\hline Cureton et al. $2007[67]$ & $1.3 \%$ & $0.35[-0.35,1.05]$ & - \\
\hline Currell et al. 2008 i [68] & $0.4 \%$ & $2.55[1.14,3.97]$ & \\
\hline Currell et al. 2008 ii [68] & $0.2 \%$ & $4.37[2.37,6.37]$ & \\
\hline Davis et al. $2000[69]$ & $0.8 \%$ & $0.49[-0.51,1.49]$ & 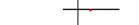 \\
\hline Desbrow et al. 2004 [70] & $0.9 \%$ & $0.02[-0.90,0.95]$ & - \\
\hline Duhamel et al. 2007 [71] & $1.2 \%$ & $0.85[0.10,1.60]$ & \\
\hline El-Sayed et al. $1995[72]$ & $0.8 \%$ & $0.57[-0.38,1.52]$ & \\
\hline Finger et al. $2018[74]$ & $1.1 \%$ & $0.04[-0.73,0.81]$ & - \\
\hline Fulco et al. 2007 i [75] & $0.7 \%$ & $0.58[-0.48,1.64]$ & \\
\hline Fulco et al. 2007 ii [75] & $0.7 \%$ & $0.43[-0.62,1.48]$ & \\
\hline Glace et al. $2019 i[78]$ & $0.9 \%$ & $0.28[-0.60,1.16]$ & \\
\hline Glace et al. 2019 ii [78] & $0.9 \%$ & $0.38[-0.51,1.26]$ & \\
\hline Glace et al. 2019 iii [78] & $0.9 \%$ & $0.38[-0.51,1.26]$ & \\
\hline Glace et al. 2019 iv [78] & $0.9 \%$ & $-0.02[-0.89,0.86]$ & - \\
\hline Gui et al. 2017 [80] & $1.0 \%$ & $0.47-0.38,1.32]$ & \\
\hline Heesch et al. $2014 \mathrm{i}[81]$ & $0.8 \%$ & $0.28[-0.71,1.26]$ & \\
\hline Hulston \& Jeukendrup 2008 [82] & $0.9 \%$ & $0.50[-0.40,1.39]$ & - \\
\hline Hulston \& Jeukendrup 2009 [83] & $0.8 \%$ & $1.35[0.36,2.35]$ & $\ldots$ \\
\hline Iny et al. $1983[84]$ & $0.8 \%$ & $0.86[-0.07,1.78]$ & \\
\hline Jarvis et al. 1999 [85] & $0.9 \%$ & $0.08[-0.79,0.96]$ & - \\
\hline Jeukendrup et al. 1997 [86] & $1.4 \%$ & $0.54[-0.11,1.19]$ & - \\
\hline Kang et al. $1995[87]$ & $\begin{array}{l}0.7 \% \\
0.7 \%\end{array}$ & $0.61[-0.47,1.69]$ & - \\
\hline Kang et al. $1996[88]$ & $0.7 \%$ & $0.48[-0.59,1.55]$ & - \\
\hline Khanna \& Manna $2005[89]$ & $0.6 \%$ & $2.10[0.96,3.24]$ & - \\
\hline King et al. $2018 \mathrm{i}[90]$ & $\begin{array}{l}0.9 \% \\
0.9 \%\end{array}$ & $\begin{array}{l}2.10[0.96,3.24] \\
0.036,1,32]\end{array}$ & - \\
\hline King et al. 2018 ii [90] & $0.9 \%$ & $0.19[-0.69,1.07]$ & — \\
\hline King et al. 2018 ii [90] & $0.9 \%$ & $0.83[-0.10,1.75]$ & . \\
\hline
\end{tabular}

Figure 8. Cont. 


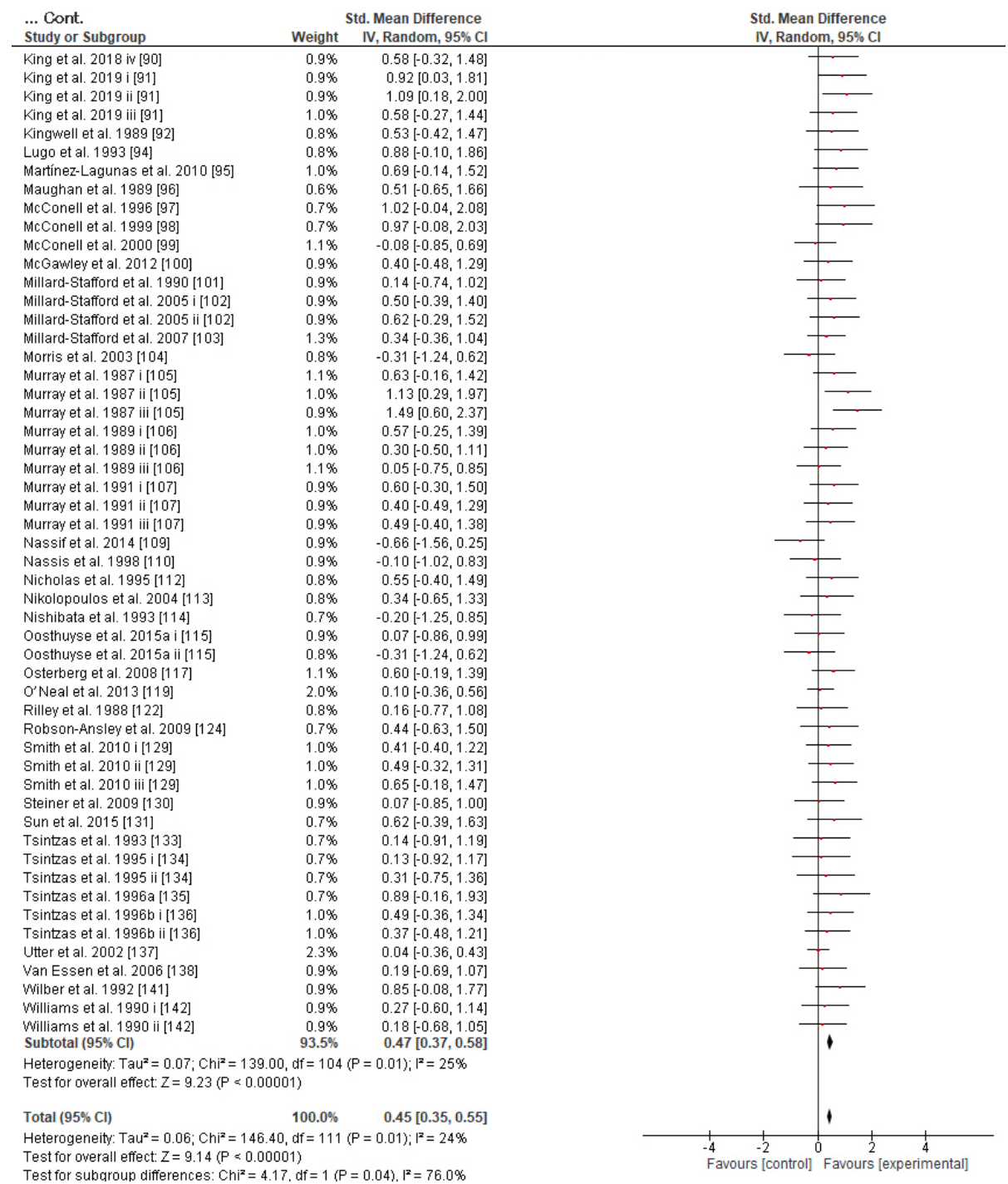

Figure 8. Forest plot shows the effects (red square symbol) of experimental carbohydrate supplementation as compared to a control on exercise outcome for 112 interventions [49-53,55-76,78,80-92,94-107,109,110,112-115,117-119,122,124,129-139,141,142]. Subgroup analyses show the results with regards to supplementation in two carbohydrate administration time groups (prior to or at the beginning of exercise, during exercise). The black diamond symbol at the subgroups and at the bottom of the figure represents the standardized mean difference with the $95 \%$ confidence intervals for all interventions following random effects meta-analyses. Studies or trials that provided insufficient data for subgroup classification were not included. Abbreviations: $\mathrm{CHO}$, carbohydrate; $\mathrm{CI}$, confidence interval; IV, inverse variance; SD, standard deviation; Std, standardized; i-vi denote different intervention arms (trials) within the same study.

\subsection{Risk of Bias}

Evidence of some risk of bias was found in all included studies (Figure 2). The predominant risk of bias derived from the lack of reliability and validity of measurements in the studies (Figure 2). Risk of bias also derived from the subjects' inclusion and statistical power process in the vast majority of the included studies (Figure 2). Furthermore, the Egger's test of the intercept and inspection of the funnel plot suggested no potential publication bias (Figure S16). 


\section{Discussion}

More than 45 years of research into the effects of $\mathrm{CHO}$ supplementation on performance during endurance exercise has provided a wealth of evidence to suggest that $\mathrm{CHO}$ contributes to fatigue postponement; yet, there have been no clear conclusions about the optimum administration timing, dosage, and type of $\mathrm{CHO}$. Meta-analyzing the efficacy of $\mathrm{CHO}(\leq 20 \%)$ solution compared with control (placebo) on prolonged exercise performance in subjects over 18 years old found that regardless of the $\mathrm{CHO}$ supplementation time administration, form, and concentration, and exercise type, mode, or protocol, consumption of $\mathrm{CHO}$ solution has positive effects on performance. However, a crossover interaction between SMD and the subjects' CRF level could exist. Carbohydrate solution seems to have a tendency to favor cyclists' performance more than that of runners. As the exercise duration increases from 1 up to $4 \mathrm{~h}$, the effect size of $\mathrm{CHO}$ intake on performance is also increased. A 6-8\% CHO solution composed of two CHOs (GL:FRU) than more components appeared sufficient to increase the chances of a better performance. Interval frequency administration during the event proved superior to administration before the exercise. Moreover, though the effect size on performance seems to be unaffected by the use of different $\mathrm{CHO}$ doses schedules, it was found that a CHO dose of $80-100 \mathrm{~g} \cdot \mathrm{h}^{-1}$ has a significantly greater effect size on endurance performance in comparison to a $\mathrm{CHO}$ dose of more than $100 \mathrm{~g} \cdot \mathrm{h}^{-1}$.

\subsection{Effects of CHO Supplementation on Endurance Exercise and Gastrointestinal Symptoms}

In 2011, two meta-analyses reported a significant acute effect of CHO supplementation on endurance exercise performance (time trials or time to exhaustion tests) $[143,144]$. In the first one, the mean effect size of $\mathrm{CHO}$ solution intake on performance, derived from 50 studies, ranged from 0.30 to 0.53 [144]; in the second one, it derived from 73 studies (122 trials) and ranged from $\sim 2$ to $\sim 6 \%$ [143]. Three years later, another meta-analysis demonstrated a positive influence on endurance performance in $82 \%$ of the 61 studies included ( $n=679$ subjects) and no effect in the remaining 18\% [145]. Another systematic review, also comparing $\mathrm{CHO}$ solution(s) with placebo or water conditions, showed an improvement of exercise performance in 13 of the 17 studies included [146]. Similarly, we found strong evidence supporting that $\mathrm{CHO}$ solutions favor performance in prolonged exercise in $88.9 \%$ of the trials included, with an overall SMD $=0.43,(n=1560)$.

On the other hand, high concentrated $\mathrm{CHO}$ sport solutions $(>8 \% \mathrm{CHO}$ ) may in some cases impede the process of fluid absorption, hinder performance, [147-150], and additionally cause unpleasant GI distress [151,152]. It has been observed that GI symptoms are associated with endurance exercise after $\mathrm{CHO}$ feeding mainly in long-distance running and triathlon [153,154]. Mesenteric blood flow is reduced when exercise intensity is high, and notably when participants are hypohydrated. This is thought to be one of the main causes of the development of GI symptoms among other physiological, mechanical, psychological, or nutritional factors $[155,156]$. These GI symptoms might impede performance, making it difficult for athletes to win or even to follow the race $[155,156]$. In this review, we found three trials in which GI problems were reported during running only; one of them did not use FRU [134] and the other two used FRU in combination [134,135]. Fructose seems to be absorbed slowly from the intestinal tract and may be responsible for a significant osmotic effect in the intestines, which may cause GI problems [157]. This may have been the case in the above studies; however, there is insignificant evidence to further investigate this hypothesis in this study, as the causal mechanisms for most GI problems are still unclear. Until this is resolved, it may be wise for the athlete to avoid single FRU as a CHO supplement.

\subsection{Effects of Mode, Protocol, and Type of Exercise}

$\mathrm{CHO}$ supplementation supposedly has different effects on performance in different modes of exercise. This may be due to different muscle groups at work (for instance, during cycling, the upper body muscles do not contribute as much as in a triathlon or cross-country 
skiing) or to possible differences in the $\mathrm{CHO}$ absorption rate, which may be aided by small stomach movements during different exercise modes (for instance, running vs. cycling). Undoubtedly, the athletes' weight is one of the determinant factors of energy expenditure, running economy, and, consequently, endurance exercise performance [158]. As running is less mechanically efficient than cycling, runners during given mechanical work exercise would be expected to have greater energy requirements than cyclists; therefore, a runner's performance would be more $\mathrm{CHO}$ dependent [159]. This simply means that when both athletes ingest the same amount of $\mathrm{CHO}$ supplements, the cyclist may have a greater advantage in exercise performance compared to the runner, under similar work exercise conditions. It has been reported, however, that the ergogenic action of exogenous $\mathrm{CHO}$ during exercise, cycling or running, although depending on different physiological and metabolic mechanisms, is similar and of a comparably relative intensity; exogenous $\mathrm{CHO}$ oxidation rates between long running and cycling are identical as well [160,161]. Our findings show that $\mathrm{CHO}$ supplementation in cyclists tends to have a larger effect size on performance in comparison to runners (Figure S5). These results may be explained by what was mentioned above; however, this topic requires further investigation. The lack of an adequate number of comparative studies on other exercise modes prevents us from drawing conclusions.

Previous studies have demonstrated that fatigue during prolonged exercise in subjects fed CHO supplement occurs at approximately the same time as muscle glycogen depletion [162-166]. On the contrary, there seems to be no dose-response association between $\mathrm{CHO}$ uptake and improved exercise capacity [160]. However, our results indicate that the effect size of $\mathrm{CHO}$ supplementation on performance has no significant difference between capacity and performance tests (Figure S6). It must be noted, by the way, that performance tests better simulate real-world competitive endurance sports and are more reliable [167]. Therefore, the effects of $\mathrm{CHO}$ supplementation on performance between time trial and time to exhaustion tests seem identical and are in agreement with other relevant systematic reviews $[144,145]$.

Originally, most of the studies investigated the role of $\mathrm{CHO}$ supplements during continuous (rather than intermittent) endurance exercise and showed an increase in endurance efficiency. During the prolonged intermittent and intermittent shuttle exercise (periods of intermittent bursts of high or lower intensity exercise (punctuated by rest or lower intensity activities)), energy is derived conjointly from anaerobic and aerobic metabolism $[8,168]$. So, in conditions of prolonged multi-sprint activities, the intra-muscular phosphocreatine and muscle glycogen stores are gradually depleted, and deterioration of performance is unavoidable $[8,168,169]$. Consequently, the research community has relatively recently extended its interest to investigating $\mathrm{CHO}$ supplements on performance during various intermittent exercise disciplines, especially in popular team games $[8,168,169]$. Nevertheless, the multi-complex nature of 'stop and start' sports (which makes it hard not only to simulate them but also to measure the subjects' physical performance (i.e., for non-technical skills) in a reliable and accurate manner) and the various methodological approaches applied among studies create conditions that complicate an interpretation of the results. The $\mathrm{CHO}$ concentration of sport solution that was used so far in these studies was $\sim 6-8 \%$ and the quantity of $\mathrm{CHO}$ (GL, SUC, and / or MD) ingested was $\sim 30-60 \mathrm{~g} \cdot \mathrm{h}^{-1}$ [169]. Based on the relevant studies, for 'stop and start' sports (lasting from 1 to $2.5 \mathrm{~h}$ ) that have shown an improved performance (or no effect), consumption of $\mathrm{CHO}$ at rates of 30-60 g.h $\mathrm{h}^{-1}$ as an acute fueling strategy is generally recommended $[8,9,169]$. Similarly, we originally found strong evidence that $\mathrm{CHO}$ intake solutions have a significant and similar impact on performance during prolonged intermittent, intermittent shuttle, and continuous endurance exercise (Figure S7). However, the positive effect of $\mathrm{CHO}$ intake solutions on intermittent shuttle exercise ( $60 \mathrm{~min}<\mathrm{T} \leq 120 \mathrm{~min}$ ) in the included studies derived from trials that had used mainly single-source $\mathrm{CHO}$ solutions (MD or SUC at a concentration range of $6.4-7 \%$, [48,49,63,79]), with only one of them having used a $\mathrm{CHO}$ dose intervention (SUC $32.6 \mathrm{~g} \cdot \mathrm{h}^{-1}$ using a 7\% solution, [79]). It is also worth mentioning here that in the three 
trials that had used $>8 \%$ concentrated solutions in a range of CHO doses $\sim 40-120 \mathrm{~g} \cdot \mathrm{h}^{-1}$, no positive result was found $[76,108]$.

\subsection{Effects of CHO Supplementation and Exercise Duration}

Theoretically, $\mathrm{CHO}$ availability via the blood is not limited during exercise in individuals taking $\mathrm{CHO}$ supplements. Our findings show that: (a) as exercise duration increases up to $4 \mathrm{~h}$, so does the effect size of $\mathrm{CHO}$ intake on performance (Figure 4); and (b) the positive SMD remains significant in exercise lasting more than $4 \mathrm{~h}$; however, the effect is tempered. As muscle glycogen is gradually reduced during a 1-4-h all-out endurance exercise, the positive impact of $\mathrm{CHO}$ intake on exercise performance increases. As the duration of the exercise is prolonged $(>4 \mathrm{~h}$ ) and the intensity inevitably decreases, the performance should depend less on the availability of CHOs [19], since the percentage of energy contribution of $\mathrm{CHO}$ will be tempered in comparison to fats [170-173]. Thus, it is not surprising that most athletes (subjects) consume less CHO $\left(\sim 20-40 \mathrm{~g} \cdot \mathrm{h}^{-1}\right)$ than the guidelines suggest [9] in endurance races lasting from 4 to $24 \mathrm{~h}$ [174-178]. The positive effect of $\mathrm{CHO}$ intake on performance in exercise lasting $45 \mathrm{~min}$ to $1 \mathrm{~h}$ appears to also be tempered in the included studies (Figure 4) (although a 6.4\% CHO solution administration showed better results, $[60,113,126-128])$. Possible explanations are that (a) muscle glycogen is not fully depleted in this duration [18], (b) muscle fatigue is possibly due to the accumulation of $\mathrm{H}_{+}[19]$, and (c) the ergogenic effect of $\mathrm{CHO}$ solution may not be exclusively metabolic but could also be attributed to the CNS [7]. Similar evidence was also reported in one meta-analysis and one systematic review [144,145].

\subsection{Effects of CRF and Gender on CHO Supplementation}

Given that (a) GL expenditure is closely correlated with exercise intensity [179], (b) training reduces the flux of GL (so athletes may rely more on fat catabolism) for a specific power output [179], (c) endurance preparation reduces endogenous oxidation of blood glucose during prolonged exercise [180], (d) trained athletes could resynthesize better GL from lactate in comparison to untrained ones [11,181], and (e) muscle glycogen has a direct impact on $\mathrm{CHO}$ availability and CRF [182], it could be assumed that the more the athletes trained, the less they depend on exogenous $\mathrm{CHO}$ during prolonged exercise $[11,179,183]$. Indeed, we observed an interaction between SMD and the subjects CRF level (Figure 3 and Figure S4). Two previous reviews had partially discussed the potentially limited effect of exogenous $\mathrm{CHO}$ on the performance of trained endurance athletes as opposed to untrained ones $[160,182]$ without reaching a permanent conclusion. The present study is probably the first one to report clear evidence as the fixed effect analysis indicated; nonetheless, more research is needed on this topic, since the conservative random effect analysis failed to prove any $\mathrm{CRF}$ effect on the efficacy of $\mathrm{CHO}$ intake during exercise (Figure 3).

In terms of sex, the already existing guidelines often assumed a similarly acute exercise effect of $\mathrm{CHO}$ supplementation in males and females. A study with a mixed-gender group [184] revealed that males and females responded in a similar fashion to $\mathrm{CHO}$ supplementation enriched with protein. On the contrary, it has been well documented that females oxidize more fat during endurance exercise than males and seem to metabolize endogenous $\mathrm{CHO}$ in different degrees, a process influenced by estrogen levels [12,52,185-188]. Our findings indicate no different effects of $\mathrm{CHO}$ supplementation on endurance performance between male (111 trials) and female subjects ( 8 trials) (Figure S2). However, it should be noted that in two of the trials, the menstrual status of the female participants was not controlled $[78,139]$. The well-established sexual dimorphism in muscle mass, hemoglobin concentration, level of reproductive hormones, and CHO oxidation [185,189-191], as well as the limited number of trials with female-only populations do not allow us to draw safe comparative conclusions. 


\subsection{Effect of Different CHO Supplement Concentrations and CHO Solution Formulations}

The energy-dependent sodium-glucose link transporter (SGLT1) actively transports GL (which may also derive from other hydrolyzed $\mathrm{CHO}$ sources, such as starch, MAL, MD, or GL-polymer) and GAL through the intestinal mucosa [7,192-194]. Other CHOs, such as FRU and SUC, use GLUT5 (facilitated diffusion) and SCRT (if SUC is not hydrolyzed into GL and FRU) protein transporters, respectively [7,192-194]. Between GL-only and FRUonly (i.e., single-source CHO solution formulation), FRU has been theorized to be a better source of $\mathrm{CHO}$ than GL because it is absorbed more slowly than GL and therefore does not stimulate an insulin response $[7,195,196]$. Accordingly, the oxidation rate of ingested FRU during endurance exercise is slower than GL [197-199] or similar [200]. With the exception of FRU, GAL, IsoMAL, and starches (oxidation rate up to $40 \mathrm{~g} \cdot \mathrm{h}^{-1}$ ) [201], exogenous $\mathrm{CHO}$ is oxidized at a maximum rate of approximately $1 \mathrm{~g} \cdot \mathrm{min}^{-1}\left(60 \mathrm{~g} \cdot \mathrm{h}^{-1}\right)[7,202-205]$. Glucose-polymer has also been shown to be more digestible, with faster gastric emptying than GL, and could hence provide GL and fluids more effectively [206-210]. Moreover, a combination of different types of CHO like GL:FRU in a ratio of 2:1 (referred to as MTC, because they use multiple protein transporters (SGLT1 and GLUT5) [211]) speeds up oxidation rates up to $25 \%$ more than was previously thought (due to the GL barrier of $60 \mathrm{~g} \cdot \mathrm{h}^{-1}$ ). A series of studies also demonstrated even higher oxidation rates, up to $75 \%$, using MTC (GL:FRU in ratio of 2:1) [201,212]. Moreover, it seems that ingestion of a solution composed of MTC during prolonged exercise $(\geq 2-2.5 \mathrm{~h})$ at high rates $\left(>60 \mathrm{~g} \cdot \mathrm{h}^{-1}\right)$ benefits performance more than the consumption of sport solutions containing GL-only (or MD-only) and minimizes the chances of GI discomfort [7,68,213-216].

The main goal of the intake of $\mathrm{CHO}$ sport solution during exercise is euhydration and euglycemia maintenance, and the minimum GL concentration required to boost water absorption is $0.9 \%$ [217]. So, in order to sustain an adequate glycogen supply and prevent hypoglycemia in exercise events lasting $>1 \mathrm{~h}$, a $6-8 \% \mathrm{CHO}$ solution was generally recommended in many reviews $[12,146,218,219]$. Briefly, most modern reviews give more appreciation to solutions of $6-8 \% \mathrm{CHO}$ concentration than to less or more concentrated drinks, and more credit to solutions composed of MTC than to single-source CHO solutions (e.g., GL or MD-only), in order to achieve high oxidation rates $\left(>90 \mathrm{~g} \cdot \mathrm{h}^{-1}\right)$, and consequently ensure better performance during prolonged exercise $[7,9,12,143,145,146,216,218-221]$. Similarly, the present meta-analysis provides evidence that $\mathrm{CHO}$ supplementation composed of GL:FRU is superior to other MTC compositions (fixed effect analysis; Figure S14). According to our results, the $6-8 \% \mathrm{CHO}$ concentrated solutions show the highest SMD (Figure S8), and the double-source $\mathrm{CHO}$ solutions increase performance significantly more than tripleor-more-source $\mathrm{CHO}$ solutions (Figure 6). These findings combined show that a $6-8 \%$ $\mathrm{CHO}$ concentrated solution composed of two types of CHO (GL:FRU in a ratio of 2:1) is sufficient to increase the chances of a better performance. This statement has to be considered with caution since the effect of GL:FR on performance, in the present study, becomes weak as far as the random effect analysis is performed (Figure 5). It appears that further investigation is needed. With regard to interventions of various single-source CHO-only solutions (53 trials), although no significant differences in efficacy were found (Figure S10), the vast majority of trials (49) appear to have used MD-only, GL-only, or SUC-only solutions, which also showed greater SMD values in comparison to MAL-only, FRU-only, or GAL-only solutions (4 trials) [145]. This is obviously the reason why in some reviews, GL, MD, and SUC are recommended in cases where a single-source $\mathrm{CHO}$ solution is preferred $[145,169]$.

\subsection{Effect of $\mathrm{CHO}$ Administration Time and Dose}

Regarding $\mathrm{CHO}$ administration time (i.e., prior to vs. during vs. late in exercise), there seems to be a lack of relevant comparative studies and only a few reviews have addressed this topic, albeit not extensively [12,160]. Briefly, it is reported that interval CHO administration during exercise is prioritized over feeding prior to exercise on its own [12]. Given that we did not study the $\mathrm{CHO}$ administration time pattern (e.g., feeding every 
$10 \mathrm{~min}$ vs. $20 \mathrm{~min}$ vs. $30 \mathrm{~min}$ vs. $40 \mathrm{~min}$ ), our results reveal the advantage of interval CHO ingestion during exercise for performance as opposed to administration prior to exercise on its own (Figure 8). However, a prior-to-exercise $\mathrm{CHO}$ feeding protocol also has a significant effect on performance that cannot be neglected, and it could be used as an added strategy or on its own as long as it does not cause gastrointestinal discomfort or provoke insulin disturbance and therefore possible rebound hypoglycemia (Figure S12).

Concerning the $\mathrm{CHO}$ dose-response, our results show that the effect size on performance is unaffected by the use of different $\mathrm{CHO}$ dose schedules $\left(\mathrm{g} \cdot \mathrm{h}^{-1}\right)$, probably because of the large variance of SMD among different trials that used different experimental methods (Figure S9). It also appears that performance is benefited significantly more from a $\mathrm{CHO}$ dose of $80-100 \mathrm{~g} \cdot \mathrm{h}^{-1}$ in comparison to a CHO dose $>100 \mathrm{~g} \cdot \mathrm{h}^{-1}$ (Figure 7 ), and also more in comparison to $60-80 \mathrm{~g} \cdot \mathrm{h}^{-1}$, though not significantly $(p=0.15)$. On the other hand, some reviews consider that $\mathrm{CHO}$ intake of up to $60 \mathrm{~g} \cdot \mathrm{h}^{-1}$ for exercise lasting up to $2.5 \mathrm{~h}$ and up to $90 \mathrm{~g} \cdot \mathrm{h}^{-1}$ when the exercise duration exceeds $2.5 \mathrm{~h}$ should be recommended $[7,9,220]$. For the most part, these recommendations seem based on a previous review [221], which in turn seems to have premised its arguments on four previous studies about trained male endurance cyclists only $[68,129,215,222]$. The first study $(N=8)$ did not actually test the dose-response in terms of $\mathrm{CHO} \mathrm{g} \cdot \mathrm{h}^{-1}$ intake but tested the $\mathrm{CHO}$ ingestion at a rate of $1.8 \mathrm{~g} \cdot \mathrm{min}^{-1}$ during $120 \mathrm{~min}$ of exercise where the significant effect was confirmed [68]. The second study $(N=9)$ compared the effect of two different iso-caloric beverages, containing GL or GL:FRU (no placebo/control group), at an ingestion rate of $2.4 \mathrm{~g} \cdot \mathrm{min}^{-1} \sim 144 \mathrm{~g} \cdot \mathrm{h}^{-1}$ on exercise lasting more than $3 \mathrm{~h}$ [215]. The third study $(N=12)$ compared ingestion of a placebo or $\mathrm{CHO}$ at doses of 15,30 , and $60 \mathrm{~g} \cdot \mathrm{h}^{-1}$ (but not a higher dose, such as 80 or $90 \mathrm{~g} \cdot \mathrm{h}^{-1}$ ) on exercise lasting more than $2 \mathrm{~h}$ [129]. The last study (in an abstract form at that time, $N=51$ ) suggested an $\mathrm{CHO}$ ingestion dose between 60 and $80 \mathrm{~g} \cdot \mathrm{h}^{-1}$ for an optimum performance enhancement when exercise lasts $2 \mathrm{~h}$ [222]. Based on this evidence, it is more reasonable to suggest a CHO ingestion dose of $60-80 \mathrm{~g} \cdot \mathrm{h}^{-1}$ for exercise lasting up to $2.5 \mathrm{~h}$ in the trained male cyclists population only, rather than recommend a CHO intake up to $60 \mathrm{~g} \cdot \mathrm{h}^{-1}$ for exercise lasting up to $2.5 \mathrm{~h}$ and up to $90 \mathrm{~g} \cdot \mathrm{h}^{-1}$ when the duration of exercise exceeds $2.5 \mathrm{~h}$ for the general population in various exercise modes.

In 2013, Jekendrup (one of the leading researchers in the field) argued that, with a few exceptions, decent dose-response research was notably absent and many of the published reports had substantial methodological flaws, which made it difficult to establish a valid relationship between the quantity of $\mathrm{CHO}$ consumption and the efficiency of the dose-response [223]. In the absolute sense of the dose-response term (i.e., $\mathrm{g} \cdot \mathrm{h}^{-1}$ ), relevant research studies are still limited at the present time [169]. We only found a total of 11 relevant studies that satisfied our inclusion criteria (Figure S9). Regardless of this, most modern reviews recommend a $\mathrm{CHO}$ intake up to $60 \mathrm{~g} \cdot \mathrm{h}^{-1}$ during an endurance event lasting up to $2.5 \mathrm{~h}$, and up to $90 \mathrm{~g} \cdot \mathrm{h}^{-1}$ when the duration of exercise exceeds $2.5 \mathrm{~h}[7,9,143,220]$. Nevertheless, as the duration of an all-out endurance exercise decreases and the intensity increases accordingly, performance should depend more on the availability of CHOs, since CHOs will contribute to a higher energy percentage than fats [19,170-173]. Therefore, much more energy would need to be derived from $\mathrm{CHO}$ sources as a percentage in an exercise lasting up to $2.5 \mathrm{~h}$ than when the exercise exceeds $2.5 \mathrm{~h}$ [170]. A CHO ingestion dose of $\sim 80 \mathrm{~g} \cdot \mathrm{h}^{-1}$ has also been suggested for an optimum performance benefit when exercise lasts $\sim 2 \mathrm{~h}$ [224]. So, in endurance events lasting up to $2.5 \mathrm{~h}$, if we supply $90 \mathrm{~g} \cdot \mathrm{h}^{-1}$ of MTC (e.g., GL: $60 \mathrm{~g} \cdot \mathrm{h}^{-1}$ and FRU: $30 \mathrm{~g} \cdot \mathrm{h}^{-1}$, taking advantage of both different CHO transporters, SGLT1 and GLUT5, respectively), theoretically, the chance of an optimum $\mathrm{CHO}$ oxidation rate [225] will be increased [226], thus increasing the chances of a higher performance in comparison to $60 \mathrm{~g} \cdot \mathrm{h}^{-1} \mathrm{GL}$-only (or MD-only) ingestion [227]. In this case, if the extra CHOs are not fully oxidized in the first $2.5 \mathrm{~h}$ of the endurance task, the residual CHOs will probably help reduce the overall amount of CHOs needed for replenishment in the later stages of the exercise or post exercise and may favor the athlete for upcoming exercise events series. Consequently, with our findings in mind, since GI comfort is not 
compromised [216,228], the recommended CHO dose could also be raised up to $90 \mathrm{~g} \cdot \mathrm{h}^{-1}$ (instead of $60 \mathrm{~g} \cdot \mathrm{h}^{-1}$ ) composed of MTC for endurance events lasting 1-2.5 $\mathrm{h}[10,90,145,146]$.

The main reason for recommending a CHO dose up to $90 \mathrm{~g} \cdot \mathrm{h}^{-1}\left(\right.$ instead of $60 \mathrm{~g} \cdot \mathrm{h}^{-1}$ ) when exercise duration exceeds $2.5 \mathrm{~h}$ is to compensate for the metabolic demands due to the increased glycogen depletion rates $[7,9,143,220]$. However, this guideline is very general and may not consider all the multifactorial metabolic demands of various ultraexercise events, as (a) most guidelines do not take into account body mass (BM) and sex differences. (b) Controversially, the above recommendation equates in theory the energy requirements in exogenous $\mathrm{CHOs}$ for endurance races that just last $>2.5$ to those for races that last, for instance, 4, 8, 12, and $24 \mathrm{~h}$, which obviously have different intensities and metabolic demands. As the duration of an ultra-endurance event increases and the intensity decreases accordingly, the performance should depend less on the availability of exogenous CHOs $[19,170-173]$. We found that the effect size of CHO intake on performance is smaller for an exercise duration $>4 \mathrm{~h}$ (4 trials) (Figure 3). It was also revealed that (a) runners' (subjects') consumption was surprisingly $14.93 \mathrm{~g} \cdot \mathrm{h}^{-1}$ in race durations $\sim 4.28 \mathrm{~h}$ [175]; (b) most athletes' consumption rate ranged from $0.27-0.64 \mathrm{~g} \cdot \mathrm{kg}^{-1} \cdot \mathrm{h}^{-1}$ in race durations $\sim 24 \mathrm{~h}$ [174] or 20-40 $\mathrm{g} \cdot \mathrm{h}^{-1}$ in single-stage and multistage ultramarathon events [176-178]; and (c) mean consumption was $62.2 \mathrm{~g} \cdot \mathrm{h}^{-1}$ in race durations of $24 \mathrm{~h} \mathrm{[229],} \mathrm{which} \mathrm{is} \mathrm{less}$ than the prevailing recommendation. Therefore, as new evidence keeps coming up in the literature, we believe that the issue of exercise duration deserves further investigation, perhaps even a reconsideration of the hitherto proposed recommendations.

However, another question arises regarding dose: Why are the doses recommended so far expressed mainly in $\mathrm{g} \cdot \mathrm{h}^{-1}$ units and not, correspondingly, in individual $\mathrm{BM}$, as proposed [9], given that heavier athletes consume more energy than lighter ones? On the one hand, a meta-analysis has reported that the ergogenic action of a $\mathrm{CHO}$ dose up to $80 \mathrm{~g} \cdot \mathrm{h}^{-1}$ depends on the athlete's size among other factors [144]. On the other hand, it has been concluded that that there is no evidence for expressing $\mathrm{CHO}$ dose guidelines in relation to BM because they are not correlated [201], a conclusion that was later adopted by several other reviewers $[7,9,143,220]$. This viewpoint appears to be based on the results of a series of CHO oxidation rate studies [161,230-233]. However, research in these studies was conducted in the same laboratory, on trained male cyclist subjects only (BM ranged from $\sim 58-84 \mathrm{~kg}$ ), whereas exercise performance was not tested, which limited the results' generalizability. Moreover, the subjects in these studies followed their own diet (except the last 1-2 days) before the experiment [161,230-233]. Since the CHO content of any diet is a determinant factor of the gut absorption capacity, as later studies have shown (due to the intestinal transporters' upregulation when the amount of $\mathrm{CHO}$ intake is increased $[201,234,235])$, it should have a significant impact on the oxidation rate. In this sense, as the subjects did not experience the same diet routine, it is reasonable to assume that their gut was trained differently, and they would have different $\mathrm{CHO}$ absorption rates $[234,235]$, most probably uncorrelated to their $\mathrm{BM}$, and thus different oxidation rates. This alone should call for a re-examination of the notion of expressing $\mathrm{CHO}$ dose guidelines in $\mathrm{g} \cdot \mathrm{h}^{-1}$ values.

\subsection{Methodological Aspects, Strength, Limitations, and Suggestions for Future Research}

Though meta-analysis has been widely applied to human performance research [236-239], its results have been criticized as 'mixing apples and oranges' [240]. However, what needs to be considered is whether studies of different internal validity and methodological control (e.g., small sample size, no control group, and non-randomized) should be included, and whether they should be considered as weighty as studies with more appropriate experimental designs. For this reason, we only included controlled intervention studies, whose authors reported that they used a specific experimental method involving a comparison group in a parallel or crossover design. We also introduced a modified version of the Cochrane risk-of-bias assessment tool to assess potential limitations of the eligible studies [24]. Nevertheless, it should be emphasized that a comparison of the 
more methodologically sound studies is not sufficient on its own. The different methodologies and techniques employed by the different studies could also explain the lack of consistency in the literature. Furthermore, we attempted to adjust the methodological research characteristics employed throughout this review using specific predefined criteria; nonetheless, evidence of overall risk-of-bias in the included studies remains subjective to some extent [24]. In the current meta-analysis, the vast majority of the eligible studies used a crossover design (139 trials). We assume that the one week washout period reported by the revised studies was sufficient for subjects to recover from residual fatigue derived from the previous exercise testing session, and that the time of washout between repeated trials did not impact the key meta-analysis findings. Hence, crossover designs are not considered a threat to this study. The effect size has also been criticized in the meta-analysis technique. Hedges (1981), who introduced the unbiased effect size, suggested a solution to this, which was adapted for use in our review [36]. In an effort to further increase the homogeneity of our results and be able to draw conclusions on variant modes, durations, and types of exercise, and different classes of subjects CRF, compositions, concentrations, and administration times of $\mathrm{CHO}$ supplements, we also applied subgroup analysis. Although only studies with accessible full-text papers written in English were selected, our outcomes show no potential publication bias. It should also be stated that even though all methodological precautions were taken, the extended period (1975-2021) of studies being analyzed introduces a level of uncertainty in drawing conclusions, due to changes in the experimental methods and designs through time. Similarly, another point of introduced uncertainty in the present meta-analysis deals with the fact that performance was not measured in all studies in a uniform way. Time to exhaustion and time trail tests evaluate different physiological mechanisms and each one entails its own repeatability accuracy [32-34]. Despite this, the present study found no effect of the performance test on CHO efficacy as others also did [144,145].

Gut trainability derived from 'nutritional training' in order to train the GI tract by improving the rate of emptying and fluid absorption and thus favor exercise performance in endurance events is a known practice among endurance athletes [234,235]. However, no study reported that subjects were checked in advance, as to whether they used to ingest $\mathrm{CHO}$ supplements or a high $\mathrm{CHO}$-enriched diet for a long period before the study, which means that we do not know if the results of these studies could have been affected by subjects' gut trainability effect. To overcome this methodological flaw in future studies, all subjects will need to follow a standardized control diet for many weeks prior to the investigation period.

Most of the review studies were conducted on cycling (100 trials), in laboratory environments (133 trials) and in a male population (111 trials), making it hard to generalize to other natural sporting environments (i.e., aquatic activities), elite athletes, and the female population. Perhaps more applied research is needed to investigate the effect of $\mathrm{CHO}$ supplements on endurance exercise of different types, durations, and modes at different phases of a female's menstrual cycle [241] and in elite athletes. Further, investigation is required to assess whether $\mathrm{CHO}$ solution administration is ergogenic in ultra-exercise events of varied duration, and what is the optimal dose, type, and concentration of a $\mathrm{CHO}$ drink for enhancing performance. Ambient temperature, exercise mode, and intensity seem to differently influence the energy substrate's oxidation and metabolism [13,14]. We did not find enough studies on different hypobaric or thermal conditions combined with different $\mathrm{CHO}$ solution temperatures to draw safe conclusions; therefore, more research is needed to address this issue. In the last decade, however, there has been an emerging interest in $\mathrm{CHO}$ dose $\left(\mathrm{CHO} \mathrm{g} \cdot \mathrm{h}^{-1}\right)$ response in endurance performance, and various studies recommend $30-60 \mathrm{~g} \cdot \mathrm{h}^{-1}$ to 90 or even a surprising $120 \mathrm{~g} \cdot \mathrm{h}^{-1}[7,9,242]$. As was earlier explained, the $\mathrm{CHO}$ dose-response in exercise performance could possibly depend on the athlete's weight [144]. Since no modern study has investigated individuals' dose-response control for their BM or lean mass in conjunction with their diet, this is another future research topic that needs to be addressed. Furthermore, any recommended dose that will be derived 
from these studies should preferably be expressed in $\mathrm{CHO} \mathrm{g} \cdot \mathrm{h}^{-1} \cdot \mathrm{kg}^{-1}$ of $\mathrm{BM}$, as suggested by the American College of Sports Medicine. This will enable global scope organizations to update their recommendations and make them more suitable and accurate for a wider range of athletes' body sizes [9]. In addition, as more investigation on dose-response is required, more studies are needed to determine the potential action of different $\mathrm{CHO}$ doses on exercise performance, especially in different exercise modalities and intermittent events. The validity and reliability of the experimental protocols should be measured and reported by the researchers accordingly.

In this article, we selected and analyzed relevant papers from a specific period (1975-2021) and verified the hypothesis that CHO supplementation during prolonged exercise enhances performance or delay fatigue progression. Maintaining blood glucose concentrations and increasing $\mathrm{CHO}$ oxidation rates could be the main reasons behind such performance improvement. Fatigue is not solely located at the peripheral level, as explained by the appearance of a "metabolic endpoint", in which muscle glycogen levels are exhausted and plasma GL levels are lowered (peripheral factor) $[243,244]$. There is substantial evidence that mechanisms located at the CNS are also involved in fatigue exhibition (central factor) $[245,246]$ and it seems that the CHO ergogenic effect also has a central component independent of the metabolic one [247]. The drop in central activation during persistent muscular contraction could be caused by substrates' depletion in the CNS and/or changes in the levels of particular neurotransmitters [244]. Exercise-induced hypoglycemia has been linked to a significant reduction in voluntary activation during persistent muscle contractions [248], as well as a drop in brain GL uptake and the overall cerebral metabolic rate [249]. On the contrary, when euglycemia is maintained, the drop in CNS activation recedes [250]. Moreover, a decrease in brain GL has also been linked to the homeostatic drive to sleep [251], suggesting that it may play a role in fatigue progression, and changes in extracellular GL concentrations have been shown to have a considerable impact on serotonin release and absorption during exercise and recovery, indicating that GL plays a key role in central neurotransmission control [252]. Thus, as exercise activity duration increases (e.g., $>2 \mathrm{~h}$ ) and glycogen depletion becomes apparent, $\mathrm{CHO}$ supplementation is also expected to continue to have a significant influence on central fatigue markers [253]. However, the mechanisms that cause performance declines can interact at any level of the brain-muscle circuit, and while the literature normally distinguishes between peripheral and central fatigue, both pathways may be intertwined and the complicated relationship between peripheral and cerebral components could influence fatigue during prolonged exercise [254]. Therefore, as the effects of $\mathrm{CHO}$ supplementation on the interactive mechanisms of central and peripheral fatigue during prolonged exercise are still unclear [255], more research is needed on this complex phenomenon impacted by both central and peripheral factors.

\subsection{Practical Implications and Guidelines for the Athlete}

In the last 45 years, the undiminished interest in research on $\mathrm{CHO}$ solutions (sport drinks) has promoted our insights and sport supplementation practices. Gastrointestinal absorption, splanchnic and muscle blood flow, muscle energy uptake, and substrate oxidation have all been established as possible barriers to the potential ergogenic effects of $\mathrm{CHO}$ solutions [256]. However, with an ever-growing body of reviews on the influence of ingesting $\mathrm{CHO}$ solutions during prolonged exercise on performance, and different recommendations from a different perspective, there is the question of what constitutes substantive advice [7-11]. Moreover, many athletes have reported that is not very clear if the dose should be up to $60 \mathrm{~g} \cdot \mathrm{h}^{-1}$ for the first $2.5 \mathrm{~h}$ and then be increased to $90 \mathrm{~g} \cdot \mathrm{h}^{-1}$, or the dose should be $90 \mathrm{~g} \cdot \mathrm{h}^{-1}$ from the beginning of an endurance event when the exercise lasts more than $2.5 \mathrm{~h}$ (personal communication, in recent ultra-endurance races). There are also many who are misled by retailers, possibly due to the difficulty of interpreting the scientific data, the existence of a plethora of different guidelines, and various misconceptions $[8,257,258]$. 
Therefore, since our study does not cover the whole spectrum of research on $\mathrm{CHO}$ solution ingestion during prolonged exercise, our recommendations are inevitably limited by our findings. We also feel that it will be more appropriate to propose a more simplified version of the already existing guidelines to athletes [9], which is likely to have a greater impact on the sport population. Thus, we think that the same or an even better result could be obtained by simply recommending a dose up to $90 \mathrm{~g} \cdot \mathrm{h}^{-1}$ of two transportable CHOs (GL:FRU in a ratio of 2:1) in the form of a $6-8 \%$ CHO solution during exercise events (intermittent and continuous) lasting from 1 to $4 \mathrm{~h}$ (Figure 9). With regard to 'stop and start' sports, it seems that a 6-8\% concentrated single-source $\mathrm{CHO}$ solution (e.g., GL or MD or SUC) is sufficient not only to serve hydration purposes, but also to meet increased demands for the exogenous $\mathrm{CHO}$ supply. For exercise lasting $45 \mathrm{~min} \leq \mathrm{T} \leq 60 \mathrm{~min}, \mathrm{a} \sim 6 \% \mathrm{CHO}$ solution is recommended. Taking supplemental CHO solutions (e.g., $30-50 \mathrm{~g} \cdot \mathrm{h}^{-1}$ as proposed by the International Society of Sports Nutrition [177]) in prolonged exercise ( $>240 \mathrm{~min}$ ) may be beneficial. Nevertheless, the fact that we found only three relevant studies prevents us from making universal recommendations; therefore, athletes should test and rehearse race nutrition strategies, including precise macronutrient/fluid, quality, and amount during training sessions. It should be duly pointed out that the guidelines suggested above are based on our analysis, which mainly derived from mild environmental condition studies, in the male population and non-elite athletes, and so their generalizability is limited to the study population/ambient condition. As perceived, scientific evidence should never be ignored. However, athletes' CHO supplementation plans could be tailored to their individual preferences, responses, and tolerance to various dose strategies and needs. Athletes should also take into account the provided options for $\mathrm{CHO}$ solution ingestion in a competition event or training session $[8-12,145,146]$. Additionally, athletes should not neglect the benefit of a balanced diet, gut training, and pre-event $\mathrm{CHO}$ supplementation, and they should always be well hydrated [8-11,145,146,234]. 


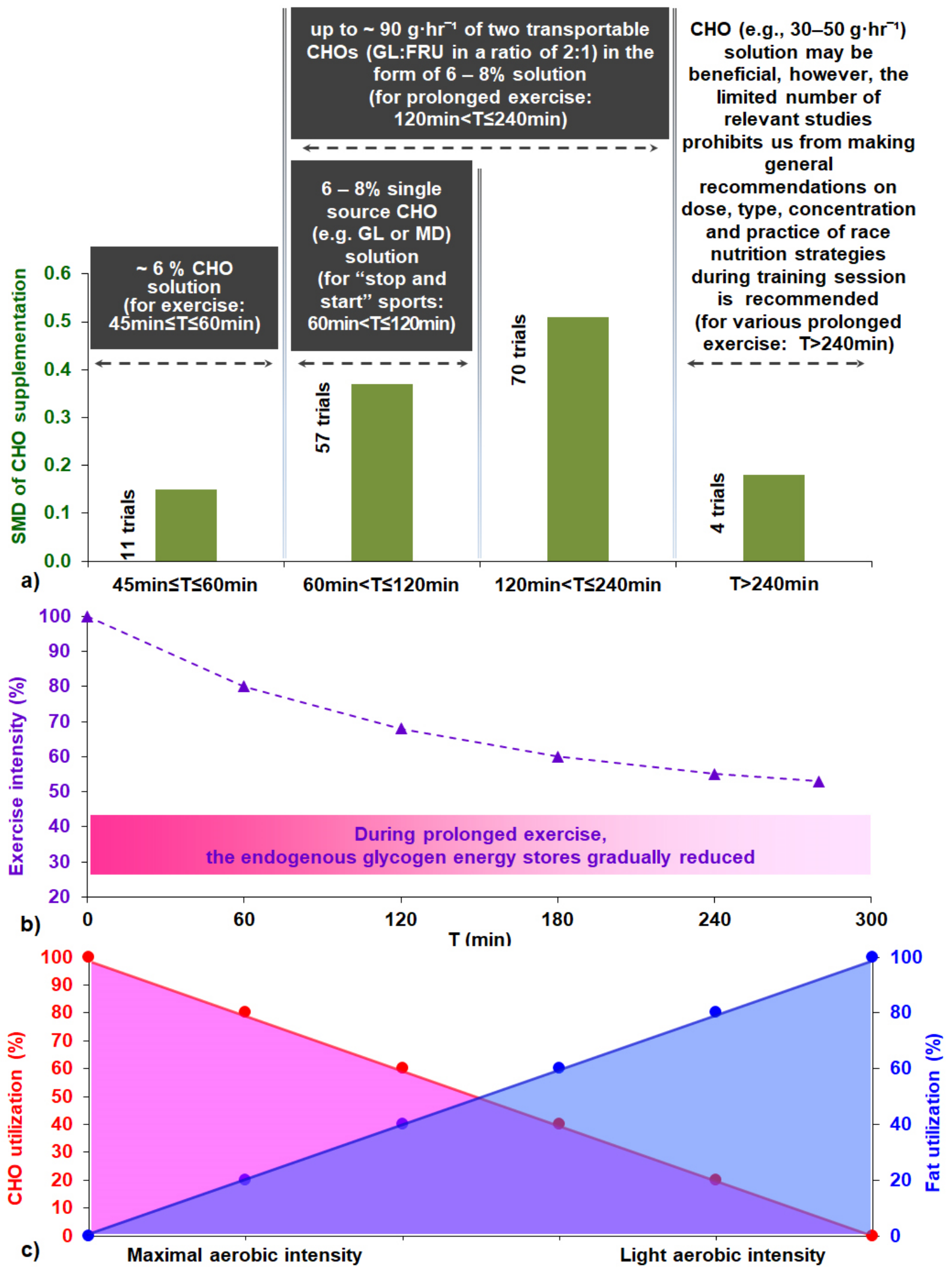

Figure 9. (a) Suggested key guidelines for carbohydrate supplementation during prolonged exercise and effect size of $\mathrm{CHO}$ supplementation as a function of exercise duration, referenced in the present study. (b) Idealized various intensities for constant exercise as a function of exercise time and endogenous glycogen energy stores reduction [170-173]. (c) Idealized fuels used as a function of exercise effort [170-173]. Abbreviations: CHO, carbohydrate; FRU, fructose; GL, glucose; MD, maltodextrin; SMD, standardized mean difference; T, exercise time. 


\section{Conclusions}

We examined the efficacy of $\mathrm{CHO}(\leq 20 \%)$ solution compared to controls on prolonged exercise performance in subjects over 18 years old in 96 studies (142 trials). We found that $\mathrm{CHO}$ solution favors performance in prolonged exercise, continuous and intermittent, and cyclists tend to have a greater effect size in comparison to runners. As the exercise duration increases from 1 to up to $4 \mathrm{~h}$, so does the effect size of $\mathrm{CHO}$ intake on performance. A crossover interaction between SMD and the subjects' CRF level was also considered, although not between gender classes; however, the number of studies in the female population are limited and the athlete's gender may be a worthy variable for future consideration. A 6-8\% CHO concentrated solution composed of two transportable CHOs (i.e., GL:FRU in a ratio of 2:1) appears to be sufficient to increase the chance of a better performance. An administration schedule during the event seems superior for performance in comparison to administration prior to exercise on its own, although $\mathrm{CHO}$ supplementation prior to exercise should not neglected. Additionally, although the effect size on performance seems to be unaffected by different $\mathrm{CHO}$ dose schedules, it appears that a CHO dose of 80-100 $\mathrm{g} \cdot \mathrm{h}^{-1}$ has a greater effect size on performance in comparison to $60-80 \mathrm{~g} \cdot \mathrm{h}^{-1}$ and is significantly more beneficial for endurance performance in comparison to a $\mathrm{CHO}$ dose $>100 \mathrm{~g} \cdot \mathrm{h}^{-1}$. However, all included studies investigating the efficacy of $\mathrm{CHO}$ supplements during prolonged exercise have shown some evidence of risk-of-bias, an issue that should be addressed in future research. More research is needed to investigate the impact of $\mathrm{CHO}$ supplementation and $\mathrm{CHO}$ dose, preferably expressed per $\mathrm{kg}$ of $\mathrm{BM}$ as suggested [9], on prolonged exercise of different types, duration, modes, in the female population, at different phases of the menstrual cycle, and in elite athletes. We hope that the findings of and questions raised by this review will help set a direction for future applied research.

Supplementary Materials: The following are available online at https://www.mdpi.com/article/ 10.3390/nu13124223/s1, Figure S1: Forest plot of comparison, experimental carbohydrate supplementation vs. control on exercise outcome including raw data (in mean and SD) and risk-of-bias judgments of all studies (and trials) for 142 interventions. Abbreviations: $C I$, confidence interval; $I V$, inverse variance; $S D$, standard deviation; $S t d$, standardized; $i-v i$ denote different intervention arms (trials) within the same study; +, low risk-of-bias; ?, some concerns risk-of-bias; - , high risk-of-bias, Figure S2: Forest plot shows the effects of experimental carbohydrate supplementation as compared to control on exercise outcome for 142 interventions. Subgroup analyses show the results with regard to the subjects' characteristics in three gender groups (Male, Female, Mixed). The black diamond symbol at the subgroups and at the bottom of the figure represents the standardized mean difference with the $95 \%$ confidence intervals for all interventions following random-effect meta-analyses. Studies or trials that provided insufficient data for subgroup classification were not included. Abbreviations: $C I$, confidence interval; $I V$, inverse variance; $S D$, standard deviation; $S t d$, standardized; $i-v i$ denote different intervention arms (trials) within the same study, Figure S3: Forest plot shows the effects of experimental carbohydrate supplementation as compared to control on exercise outcome for 140 interventions. Subgroup analyses show the results with regard to the subjects' characteristics in three age groups [Young (18-29 years), Adults I (30-39 years), Adults II (40-49 years)]. The black diamond symbol at the subgroups and at the bottom of the figure represents the standardized mean difference with the $95 \%$ confidence intervals for all interventions following random-effect meta-analyses. Studies or trials that provided insufficient data for subgroup classification were not included. Abbreviations: $C I$, confidence interval; $I V$, inverse variance; $S D$, standard deviation; $S t d$, standardized; $i-v i$ denote different intervention arms (trials) within the same study, Figure S4: Forest plot shows the effects of experimental carbohydrate supplementation as compared to control on exercise outcome for 127 interventions. Subgroup analyses show the results with regards to subjects' characteristics in four CRF groups (Superior, Excellent, Good and Fair). The black diamond symbol at the subgroups and at the bottom of the figure represents the standardized mean difference with the $95 \%$ confidence intervals for all interventions following fixed effects meta-analyses. Studies or trials that provided not sufficient data for subgroup classification were not included. Abbreviations: $C I$, confidence interval; $C R F$, cardio-respiratory fitness; $I V$, inverse variance; $S D$, standard 
deviation; Std, standardized; $i-v i$ denote different intervention arms (trials) within the same study, Figure S5: Forest plot shows the effects of experimental carbohydrate supplementation as compared to control on exercise outcome for 142 interventions. Subgroup analyses show the results with regard to exercise task in three exercise mode groups [Cycling, Running, Other (triathlon, duathlon, walking, loaded marching, roller-skiing)]. The black diamond symbol at the subgroups and at the bottom of the figure represents the standardized mean difference with the $95 \%$ confidence intervals for all interventions following random-effect meta-analyses. Studies or trials that provided insufficient data for subgroup classification were not included. Abbreviations: $C I$, confidence interval; $I V$, inverse variance; $S D$, standard deviation; $S t d$, standardized; $i-v i$ denote different intervention arms (trials) within the same study, Figure S6: Forest plot shows the effects of experimental carbohydrate supplementation as compared to control on exercise outcome for 142 interventions. Subgroup analyses show the results with regard to exercise task in two exercise protocol test groups (Capacity, Performance). The black diamond symbol at the subgroups and at the bottom of the figure represents the standardized mean difference with the $95 \%$ confidence intervals for all interventions following random-effect meta-analyses. Studies or trials that provided insufficient data for subgroup classification were not included. Abbreviations: $C I$, confidence interval; $I V$, inverse variance; $S D$, standard deviation; Std, standardized; $i-v i$ denote different intervention arms (trials) within the same study, Figure S7: Forest plot shows the effects of experimental carbohydrate supplementation as compared to control on exercise outcome for 142 interventions. Subgroup analyses show the results with regard to exercise task in three exercise type groups (Intermittent, Continuous, Intermittent shuttle). The black diamond symbol at the subgroups and at the bottom of the figure represents the standardized mean difference with the $95 \%$ confidence intervals for all interventions following random-effect meta-analyses. Studies or trials that provided insufficient data for subgroup classification were not included. Abbreviations: $C I$, confidence interval; $I V$, inverse variance; $S D$, standard deviation; Std, standardized; $i-v i$ denote different intervention arms (trials) within the same study, Figure S8: Forest plot shows the effects of experimental carbohydrate supplementation as compared to control on exercise outcome for 142 interventions. Subgroup analyses show the results with regard to supplementation in seven carbohydrate concentration groups $(0 \%<\mathrm{CHO} \leq 2 \%, 2 \%<\mathrm{CHO} \leq 4 \%$, $4 \%<\mathrm{CHO} \leq 6 \%, 6 \%<\mathrm{CHO} \leq 8 \%, 8 \%<\mathrm{CHO} \leq 10 \%, 10 \%<\mathrm{CHO} \leq 15 \%, 15 \%<\mathrm{CHO} \leq 20 \%)$. The black diamond symbol at the subgroups and at the bottom of the figure represents the standardized mean difference with the $95 \%$ confidence intervals for all interventions following random-effect meta-analyses. Studies or trials that provided insufficient data for subgroup classification were not included. Abbreviations: $C H O$, carbohydrate; $C I$, confidence interval; $I V$, inverse variance; $S D$, standard deviation; $S t d$, standardized; $i-v i$ denote different intervention arms (trials) within the same study, Figure S9: Forest plot shows the effects of experimental carbohydrate supplementation as compared to control on exercise outcome for 31 interventions. Subgroup analyses show the results with regard to supplementation in five carbohydrate dose groups (CHO dose $\leq 40 \mathrm{~g} \cdot \mathrm{h}^{-1}$, $40 \mathrm{~g} \cdot \mathrm{h}^{-1}<\mathrm{CHO}$ dose $\leq 60 \mathrm{~g} \cdot \mathrm{h}^{-1}, 60 \mathrm{~g} \cdot \mathrm{h}^{-1}<\mathrm{CHO}$ dose $\leq 80 \mathrm{~g} \cdot \mathrm{h}^{-1}$, $80 \mathrm{~g} \cdot \mathrm{h}^{-1}<\mathrm{CHO}$ dose $\leq 100 \mathrm{~g} \cdot \mathrm{h}^{-1}, \mathrm{CHO}$ dose $>100 \mathrm{~g} \cdot \mathrm{h}^{-1}$ ). The black diamond symbol at the subgroups and at the bottom of the figure represents the standardized mean difference with the $95 \%$ confidence intervals for all interventions following random-effect meta-analyses. Studies or trials that provided insufficient data for subgroup classification were not included. Abbreviations: $C H O$, carbohydrate; $C I$, confidence interval; $I V$, inverse variance; $S D$, standard deviation; Std, standardized; $i-v i$ denote different intervention arms (trials) within the same study, Figure S10: Forest plot shows the effects of experimental carbohydrate supplementation as compared to control on exercise outcome for 53 interventions. Subgroup analyses show the results with regard to supplementation in six single source carbohydrate only groups (GL, MD, SUC, MAL, FRU, GAL). The black diamond symbol at the subgroups and at the bottom of the figure represents the standardized mean difference with the $95 \%$ confidence intervals for all interventions following random-effect meta-analyses. Studies or trials that provided insufficient data for subgroup classification were not included. Abbreviations: $\mathrm{CHO}$, carbohydrate; $\mathrm{CI}$, confidence interval; $F \mathrm{RU}$, fructose; $G A L$, galactose; $G L$, glucose; $I V$, inverse variance; $M D$, maltodextrin; $M A L$, maltose; $S D$, standard deviation; Std, standardized; SUC, sucrose; $i$-vi denote different intervention arms (trials) within the same study, Figure S11: Forest plot shows the effects of experimental carbohydrate supplementation as compared to control on exercise outcome for 103 interventions. Subgroup analyses show the results with regard to supplementation in three carbohydrate solution formulation groups (Singlesource $\mathrm{CHO}$ solution, Double-source $\mathrm{CHO}$ solution, Triple-or-more-source $\mathrm{CHO}$ solution). The 
black diamond symbol at the subgroups and at the bottom of the figure represents the standardized mean difference with the $95 \%$ confidence intervals for all interventions following random-effect meta-analyses. Studies or trials that provided insufficient data for subgroup classification were not included. Abbreviations: $C H O$, carbohydrate; $C I$, confidence interval; $I V$, inverse variance; $S D$, standard deviation; Std, standardized; $i-v i$ denote different intervention arms (trials) within the same study, Figure S12: Forest plot shows the effects of experimental carbohydrate supplementation as compared to control on exercise outcome for 142 interventions. Subgroup analyses show the results with regard to supplementation in four carbohydrate administration time groups (Prior to or at the beginning, During, Prior to or at the beginning + during, Late in exercise). The black diamond symbol at the subgroups and at the bottom of the figure represents the standardized mean difference with the $95 \%$ confidence intervals for all interventions following random-effect meta-analyses. Studies or trials that provided insufficient data for subgroup classification were not included. Abbreviations: $\mathrm{CHO}$, carbohydrate; $\mathrm{CI}$, confidence interval; $I V$, inverse variance; $S D$, standard deviation; Std, standardized; $i-v i$ denote different intervention arms (trials) within the same study, Figure S13: Forest plot shows the effects of experimental carbohydrate supplementation as compared to control on exercise outcome for 21 interventions. Subgroup analyses show the results with regard to supplementation in two temperature of supplement administration groups [Cool $\left(<18{ }^{\circ} \mathrm{C}\right)$, Neutral $\left.\left(18-26^{\circ} \mathrm{C}\right)\right]$. The black diamond symbol at the subgroups and at the bottom of the figure represents the standardized mean difference with the $95 \%$ confidence intervals for all interventions following random-effect meta-analyses. Studies or trials that provided insufficient data for subgroup classification were not included. Abbreviations: $\mathrm{CHO}$, carbohydrate; $\mathrm{CI}$, confidence interval; $I V$, inverse variance; $S D$, standard deviation; $S t d$, standardized; $i-v i$ denote different intervention arms (trials) within the same study, Figure S14: Forest plot shows the effects of experimental carbohydrate supplementation as compared to control on exercise outcome for 50 interventions. Subgroup analyses show the results with regards to supplementation in twelve MTC groups (GL:FRU, GL:SUC, GL:MD, MD:FRU, MD:DEX, MD:SUC, GL:MD:FRU, GL:MD:DEX, GL:SUC:FRU, GL:MD:MAL:Saccharides, SUC:MD:IsoMAL, Unclear CHO substances mixture). The black diamond symbol at the subgroups and at the bottom of the figure represents the standardized mean difference with the $95 \%$ confidence intervals for all interventions following fixed effects meta-analyses. Studies or trials that provided not sufficient data for subgroup classification were not included. Abbreviations: $\mathrm{CHO}$, carbohydrate, $C I$, confi-dence interval; $D E X$, dextrose; $F R U$, fructose; $G A L$, galactose; $G L$, glucose; $I V$, inverse variance; $M D$, maltodextrin; $M A L$, maltose; $M T C$, multiple transportable carbohydrate; $S D$, standard deviation; Std, standardized; SUC, sucrose; $i-v i$ denote different intervention arms (trials) within the same study, Figure S15: Forest plot shows the effects of experimental carbohydrate supplementation as compared to control on exercise outcome for 102 interventions. Subgroup analyses show the results with regard to ambient conditions in two thermal condition groups $\left[\mathrm{Cool}\left(<18{ }^{\circ} \mathrm{C}\right)\right.$, Neutral $\left(18-26^{\circ} \mathrm{C}\right)$, Heat $\left.\left(>26^{\circ} \mathrm{C}\right)\right]$. The black diamond symbol at the subgroups and at the bottom of the figure represents the standardized mean difference with the $95 \%$ confidence intervals for all interventions following random-effect meta-analyses. Studies or trials that provided insufficient data for subgroup classification were not included. Abbreviations: $\mathrm{CHO}$, carbohydrate; $\mathrm{CI}$, confidence interval; $I V$, inverse variance; $S D$, standard deviation; $S t d$, standardized; $i-v i$ denote different intervention arms (trials) within the same study, Figure S16: Funnel plot of comparison: I. in all studies (and trials); and II.-VII. in classes of different subgroups analysis.

Author Contributions: Conceptualization, D.I.B.; methodology, D.I.B.; software, D.I.B.; validation, D.I.B.; A.S.; E.D.Z.; N.D.G. and A.K.T.; formal analysis, D.I.B. and A.K.T.; investigation, D.I.B. and A.K.T.; resources, D.I.B. and A.S.; data curation, D.I.B.; E.D.Z. and A.K.T.; writing-original draft preparation, D.I.B.; writing—review and editing, D.I.B.; A.S.; E.D.Z.; N.D.G. and A.K.T.; visualization, D.I.B.; supervision, D.I.B.; project administration, D.I.B. All authors have read and agreed to the published version of the manuscript.

Funding: This research received no external funding.

Institutional Review Board Statement: Not applicable.

Informed Consent Statement: Not applicable.

Data Availability Statement: All data that support the reported results can be found in the Supplements section. 
Acknowledgments: We are indebted to the independent reviewers (E.Z., G.M., A.K., K.P. and T.T.) for their enthusiastic and consistent assistance in data collection.

Conflicts of Interest: The authors declare no conflict of interest.

\section{References}

1. Zuntz, N. Ueber die Bedeutung der verschiedenen Nährstoffe als Erzeuger der Muskelkraft. Pflüg. Arch. Gesammte Physiol. Menschen Thiere 1901, 83, 557-571. [CrossRef]

2. Christensen, E.H.; Hansen, O., II. Untersuchungen über die Verbrennungsvorgänge bei langdauernder, schwerer Muskelarbeit1. Skand. Arch. Physiol. 1939, 81, 152-159. [CrossRef]

3. Bergström, J.; Hultman, E. Muscle glycogen synthesis after exercise: An enhancing factor localized to the muscle cells in man. Nature 1966, 210, 309-310. [CrossRef]

4. Bergström, J.; Hultman, E. A study of the glycogen metabolism during exercise in man. Scand. J. Clin. Lab. Investig. 1967, 19, 218-228. [CrossRef]

5. Brooke, J.D.; Davies, G.J.; Green, L.F. The effects of Normal and Glucose Syrup Work on the Performance of Racing Cyclists. J Sports Med. Phys. Fit. 1975, 15, 257-265.

6. Coyle, E.F.; Hagberg, J.M.; Hurley, B.F.; Martin, W.H.; Ehsani, A.A.; Holloszy, J.O. Carbohydrate feeding during prolonged strenuous exercise can delay fatigue. J. Appl. Physiol. Respir. Environ. Exerc. Physiol. 1983, 55, 230-235. [CrossRef]

7. Baker, L.B.; Jeukendrup, A.E. Optimal composition of fluid-replacement beverages. Compr. Physiol. 2014, 4, 575-620. [CrossRef]

8. Collins, J.; Maughan, R.J.; Gleeson, M.; Bilsborough, J.; Jeukendrup, A.; Morton, J.P.; Phillips, S.M.; Armstrong, L.; Burke, L.M.; Close, G.L.; et al. UEFA expert group statement on nutrition in elite football. Current evidence to inform practical recommendations and guide future research. Br. J. Sports Med. 2020, 55, 416. [CrossRef]

9. Thomas, D.T.; Erdman, K.A.; Burke, L.M. American College of Sports Medicine Joint Position Statement. Nutrition and Athletic Performance. Med. Sci. Sports Exerc. 2016, 48, 543-568. [CrossRef]

10. Mata, F.; Valenzuela, P.L.; Gimenez, J.; Tur, C.; Ferreria, D.; Domínguez, R.; Sanchez-Oliver, A.J.; Sanz, J.M.M. Carbohydrate availability and physical performance: Physiological overview and practical recommendations. Nutrients 2019, $11,1084$. [CrossRef]

11. Brooks, G.A. The precious few grams of glucose during exercise. Int. J. Mol. Sci. 2020, 21, 5733. [CrossRef]

12. Kerksick, C.M.; Arent, S.; Schoenfeld, B.J.; Stout, J.R.; Campbell, B.; Wilborn, C.D.; Taylor, L.; Kalman, D.; Smith-Ryan, A.E.; Kreider, R.B.; et al. International society of sports nutrition position stand: Nutrient timing. J. Int. Soc. Sports Nutr. 2017, 14, 33. [CrossRef]

13. Gagnon, D.D.; Perrier, L.; Dorman, S.C.; Oddson, B.; Larivière, C.; Serresse, O. Ambient temperature influences metabolic substrate oxidation curves during running and cycling in healthy men. Eur. J. Sport Sci. 2020, 20,90-99. [CrossRef]

14. Maunder, E.D.; Plews, D.J.; Merien, F.; Kilding, A.E. Exercise intensity regulates the effect of heat stress on substrate oxidation rates during exercise. Eur. J. Sport Sci. 2020, 20, 935-943. [CrossRef]

15. Higgins, J.P.T.; Altman, D.G.; Gøtzsche, P.C.; Jüni, P.; Moher, D.; Oxman, A.D.; Savović, J.; Schulz, K.F.; Weeks, L.; Sterne, J.A.C. The Cochrane Collaboration's tool for assessing risk of bias in randomised trials. BMJ 2011, 343, d5928. [CrossRef]

16. Liberati, A.; Altman, D.G.; Tetzlaff, J.; Mulrow, C.; Gøtzsche, P.C.; Ioannidis, J.P.A.; Clarke, M.; Devereaux, P.J.; Kleijnen, J.; Moher, D. The PRISMA statement for reporting systematic reviews and meta-analyses of studies that evaluate health care interventions: Explanation and elaboration. J. Clin. Epidemiol. 2009, 62, e1-e34. [CrossRef]

17. The Cochrane Collaboration. Cochrane Handbook for Systematic Reviews of Interventions, 2nd ed.; Higgins, J.P.T., Thomas, J., Chandler, J., Cumpston, M., Li, T., Page, M.J., Welch, V.A., Eds.; John Wiley \& Sons: Chichester, UK, 2019 ; ISBN 9781119536628.

18. Phillips, S.M.; Sproule, J.; Turner, A.P. Carbohydrate ingestion during team games exercise: Current knowledge and areas for future investigation. Sports Med. 2011, 41, 559-585. [CrossRef]

19. Coyle, E.F. Fluid and fuel intake during exercise. J. Sports Sci. 2004, 22, 39-55. [CrossRef]

20. Hopewell, S.; Dutton, S.; Yu, L.M.; Chan, A.W.; Altman, D.G. The quality of reports of randomised trials in 2000 and 2006 : Comparative study of articles indexed in PubMed. BMJ 2010, 340, c723. [CrossRef]

21. Deltsidou, A.; Zarikas, V.; Mastrogiannis, D.; Kapreli, E.; Bourdas, D.; Raftopoulos, V.; Noula, M.; Lykeridou, K. Data on advanced glycation end-products concentrations and haemodynamic parameters following caffeine and nicotine consumption in nursing students. Data Brief 2020, 32, 106063. [CrossRef]

22. Havenetidis, K.; Bourdas, D. Creatine supplementation: Effects on urinary excretion and anaerobic performance. J. Sports Med. Phys. Fit. 2003, 43, 347-355.

23. Stecker, R.A.; Harty, P.S.; Jagim, A.R.; Candow, D.G.; Kerksick, C.M. Timing of ergogenic aids and micronutrients on muscle and exercise performance. J. Int. Soc. Sports Nutr. 2019, 16, 37. [CrossRef]

24. Sterne, J.A.C.; Savović, J.; Page, M.J.; Elbers, R.G.; Blencowe, N.S.; Boutron, I.; Cates, C.J.; Cheng, H.Y.; Corbett, M.S.; Eldridge, S.M.; et al. RoB 2: A revised tool for assessing risk of bias in randomised trials. BMJ 2019, 366, 14898. [CrossRef]

25. Koes, B.W.; Assendelft, W.J.J.; Van der Heijden, G.J.M.G.; Bouter, L.M.; Knipschild, P.G. Spinal manipulation and mobilisation for back and neck pain: A blinded review. Br. Med. J. 1991, 303, 1298-1303. [CrossRef]

26. Beckerman, H.; De Bie, R.A.; Bouter, L.M.; De Cuyper, H.J.; Oostendorp, R.A.B. The efficacy of laser therapy for musculoskeletal and skin disorders: A criteria-based meta-analysis of randomized clinical trials. Phys. Ther. 1992, 72, 483-491. [CrossRef] 
27. Wood, L.; Egger, M.; Gluud, L.L.; Schulz, K.F.; Jüni, P.; Altman, D.G.; Gluud, C.; Martin, R.M.; Wood, A.J.G.; Sterne, J.A.C. Empirical evidence of bias in treatment effect estimates in controlled trials with different interventions and outcomes: Metaepidemiological study. BMJ 2008, 336, 601-605. [CrossRef]

28. Verhagen, A.P.; De Vet, H.C.W.; De Bie, R.A.; Kessels, A.G.H.; Boers, M.; Knipschild, P.G. Taking baths: The efficacy of balneotherapy in patients with arthritis. A systematic review. J. Rheumatol. 1997, 24, 1964-1971.

29. Schulz, K.F.; Altman, D.G.; Moher, D. CONSORT 2010 Statement: Updated guidelines for reporting parallel group randomised trials. J. Clin. Epidemiol. 2010, 63, 834-840. [CrossRef]

30. Moher, D.; Hopewell, S.; Schulz, K.F.; Montori, V.; Gøtzsche, P.C.; Devereaux, P.J.; Elbourne, D.; Egger, M.; Altman, D.G. CONSORT 2010 explanation and elaboration: Updated guidelines for reporting parallel group randomised trials. BMJ 2010, 340, c869. [CrossRef]

31. Current Version of RoB 2. Available online: https://sites.google.com/site/riskofbiastool/welcome/rob-2-0-tool/current-versionof-rob-2 (accessed on 24 November 2021).

32. Hopkins, W.G.; Schabort, E.J.; Hawley, J.A. Reliability of power in physical performance tests. Sports Med. 2001, 31, 211-234. [CrossRef]

33. Hopkins, W.G.; Hawley, J.A.; Burke, L.M. Design and analysis of research on sport performance enhancement. Med. Sci. Sports Exerc. 1999, 31, 472-485. [CrossRef] [PubMed]

34. Hopkins, W.G. How to Interpret Changes in an Athletic Performance Test. Sportscience 2004, 8, 1-7.

35. Thomas, J.R.; Nelson, J.K.; Silverman, S.J. Research Methods in Physical Activity, 7th ed.; Human Kinetics Publishers Inc.: Champaign, IL, USA, 2015; ISBN 9781450470445.

36. Hedges, L.V. Distribution Theory for Glass's Estimator of Effect size and Related Estimators. J. Educ. Stat. 1981, 6, 107-128. [CrossRef]

37. Hedges, L.V.; Stock, W. The Effects of Class Size: An Examination of Rival Hypotheses. Am. Educ. Res. J. 1983, 20, 85. [CrossRef]

38. Deeks, J.; Higgins, J. Statistical Algorithms in Review Manager 5; Cochrane Collaboration: London, UK, 2010 ; pp. 1-11.

39. World Health Organization. World Health Statistics 2019: Monitoring Health for the SDGs, Sustainable Development Goals; WHO/DAD/2019.1: Geneva, Switzerland, 2019; ISBN 9789241565707.

40. Heyward, V.H.; Gibson, A.L. Assessing Cardiorespiratory Fitness. In Advanced Fitness Assessment and Exercise Prescription; Human Kinetics Publishers Inc.: Champaign, IL, USA, 2014; pp. 79-119. ISBN 78-1-4504-6600-4.

41. Sedgwick, P. Meta-analyses: What is heterogeneity? BMJ 2015, 350, h1435. [CrossRef]

42. Higgins, J.P.T.; Thompson, S.G.; Deeks, J.J.; Altman, D.G. Measuring inconsistency in meta-analyses. Br. Med. J. 2003, 327, 557-560. [CrossRef] [PubMed]

43. Higgins, J.P.T.; Thompson, S.G. Quantifying heterogeneity in a meta-analysis. Stat. Med. 2002, 21, 1539-1558. [CrossRef]

44. Brockwell, S.E.; Gordon, I.R. A comparison of statistical methods for meta-analysis. Stat. Med. 2001, 20, 825-840. [CrossRef]

45. Rice, K.; Higgins, J.P.T.; Lumley, T. A re-evaluation of fixed-effect(s) meta-analysis. J. R. Stat. Soc. 2018, 181, 205-227. [CrossRef]

46. Peto, R.; Collins, R.; Gray, R. "Large-scale randomized evidence: Large, simple trials and overviews of trials": Discussion. A clinician's perspective on meta-analyses. J. Clin. Epidemiol. 1995, 48, 23-40. [CrossRef]

47. Acker-Hewitt, T.L.; Shafer, B.M.; Saunders, M.J.; Goh, Q.; Luden, N.D. Independent and combined effects of carbohydrate and caffeine ingestion on aerobic cycling performance in the fed state tiffany. Appl. Physiol. Nutr. Metab. 2012, 37, 276-283. [CrossRef] [PubMed]

48. Alghannam, A.F. Carbohydrate-protein ingestion improves subsequent running capacity towards the end of a football-specific intermittent exercise. Appl. Physiol. Nutr. Metab. 2011, 36, 748-757. [CrossRef] [PubMed]

49. Ali, A.; Williams, C.; Nicholas, C.W.; Foskett, A. The influence of carbohydrate-electrolyte ingestion on soccer skill performance. Med. Sci. Sports Exerc. 2007, 39, 1969-1976. [CrossRef]

50. Anastasiou, C.A.; Kavouras, S.A.; Koutsari, C.; Georgakakis, C.; Skenderi, K.; Beer, M.; Sidossis, L.S. Effect of maltose-containing sports drinks on exercise performance. Int. J. Sport Nutr. Exerc. Metab. 2004, 14, 609-625. [CrossRef]

51. Angus, D.J.; Hargreaves, M.; Dancey, J.; Febbraio, M.A. Effect of carbohydrate or carbohydrate plus medium-chain triglyceride ingestion on cycling time trial performance. J. Appl. Physiol. 2000, 88, 113-119. [CrossRef]

52. Bailey, S.P.; Zacher, C.M.; Mittleman, K.D. Effect of menstrual cycle phase on carbohydrate supplementation during prolonged exercise to fatigue. J. Appl. Physiol. 2000, 88, 690-697. [CrossRef]

53. Baldassarre, R.; Ieno, C.; Bonifazi, M.; Di Castro, A.; Gianfelici, A.; Piacentini, M.F. Carbohydrate supplementation during a simulated 10-km open water swimming race: Effects on physiological, perceptual parameters and performance. Eur. J. Sport Sci. 2021. ahead of print. [CrossRef]

54. Baur, D.A.; Schroer, A.B.; Luden, N.D.; Womack, C.J.; Smyth, S.A.; Saunders, M.J. Glucose-fructose enhances performance versus isocaloric, but not moderate, glucose. Med. Sci. Sports Exerc. 2014, 46, 1778-1786. [CrossRef]

55. Below, P.R.; Mora-Rodríguez, R.; José, G.A.; Coyle, E.F. Fluid and carbohydrate ingestion independently improve performance during $1 \mathrm{~h}$ of intense exercise. Med. Sci. Sports Exerc. 1995, 27, 200-210. [CrossRef]

56. Bishop, N.C.; Blannin, A.K.; Walsh, N.P.; Gleeson, M. Carbohydrate beverage ingestion and neutrophil degranulation responses following cycling to fatigue at 75\% VO2 max. Int. J. Sports Med. 2001, 22, 226-231. [CrossRef]

57. Burgess, W.A.; Davis, J.M.; Bartoli, W.P.; Woods, J.A. Failure of low dose carbohydrate feeding to attenuate glucoregulatory hormone responses and improve endurance performance. Int. J. Sport Nutr. 1991, 1, 338-352. [CrossRef] [PubMed] 
58. Byrne, C.; Lin, C.L.; Chew, S.A.N.; Ming, E.T.Y. Water versus Carbohydrate-Electrolyte Fluid Replacement during. Mil. Med. 2005, 170, 715-722. [CrossRef]

59. Campbell, C.; Prince, D.; Braun, M.; Applegate, E.; Casazza, G.A. Carbohydrate-supplement form and exercise performance. Int. J. Sport Nutr. Exerc. Metab. 2008, 18, 179-190. [CrossRef] [PubMed]

60. Carter, J.; Jeukendrup, A.E.; Mundel, T.; Jones, D.A. Carbohydrate supplementation improves moderate and high-intensity exercise in the heat. Pflug. Arch. Eur. J. Physiol. 2003, 446, 211-219. [CrossRef]

61. Carter, J.; Jeukendrup, A.E.; Jones, D.A. The effect of sweetness on the efficacy of carbohydrate supplementation during exercise in the heat. Can. J. Appl. Physiol. 2005, 30, 379-391. [CrossRef]

62. Clark, V.R.; Hopkins, W.G.; Hawley, J.A.; Burke, L.M. Placebo effect of carbohydrate feedings during a 40-km cycling time trial. Med. Sci. Sports Exerc. 2000, 32, 1642-1647. [CrossRef]

63. Clarke, N.D.; Maclaren, D.P.M.; Reilly, T.; Drust, B. Carbohydrate ingestion and pre-cooling improves exercise capacity following soccer-specific intermittent exercise performed in the heat. Eur. J. Appl. Physiol. 2011, 111, 1447-1455. [CrossRef]

64. Cole, K.J.; Grandjean, P.W.; Sobszak, R.J.; Mitchell, J.B. Effect of carbohydrate composition on fluid balance, gastric emptying, and exercise performance. Int. J. Sport Nutr. 1993, 3, 408-417. [CrossRef]

65. Cole, M.; Hopker, J.G.; Wiles, J.D.; Coleman, D.A. The effects of acute carbohydrate and caffeine feeding strategies on cycling efficiency. J. Sports Sci. 2018, 36, 817-823. [CrossRef]

66. Coletta, A.; Thompson, D.L.; Raynor, H.A. The influence of commercially-available carbohydrate and carbohydrate-protein supplements on endurance running performance in recreational athletes during a field trial. J. Int. Soc. Sports Nutr. 2013, 10, 17. [CrossRef]

67. Cureton, K.J.; Warren, G.L.; Millard-Stafford, M.L.; Wingo, J.E.; Trilk, J.; Buyckx, M. Caffeinated sports drink: Ergogenic effects and possible mechanisms. Int. J. Sport Nutr. Exerc. Metab. 2007, 17, 35-55. [CrossRef] [PubMed]

68. Currell, K.; Jeukendrup, A.E. Superior endurance performance with ingestion of multiple transportable carbohydrates. Med. Sci. Sports Exerc. 2008, 40, 275-281. [CrossRef] [PubMed]

69. Davis, J.M.; Welsh, R.S.; Alderson, N.A. Effects of carbohydrate and chromium ingestion during intermittent high-intensity exercise to fatigue. Int. J. Sport Nutr. 2000, 10, 476-485. [CrossRef] [PubMed]

70. Desbrow, B.; Anderson, S.; Barrett, J.; Rao, E.; Hargreaves, M. Carbohydrate-electrolyte feedings and $1 \mathrm{~h}$ time trial cycling performance. Int. J. Sport Nutr. Exerc. Metab. 2004, 14, 541-549. [CrossRef]

71. Duhamel, T.A.; Green, H.J.; Stewart, R.D.; Foley, K.P.; Smith, I.C.; Ouyang, J. Muscle metabolic, SR Ca2+-cycling responses to prolonged cycling, with and without glucose supplementation. J. Appl. Physiol. 2007, 103, 1986-1998. [CrossRef]

72. El-Sayed, M.S.; Rattu, A.J.M.; Roberts, I. Effects of carbohydrate feeding before and during prolonged exercise on subsequent maximal exercise performance capacity. Int. J. Sport Nutr. 1995, 5, 215-224. [CrossRef]

73. El-Sayed, M.S.; Balmer, J.; Rattu, A.J.M. Carbohydrate ingestion improves endurance performance during a $1 \mathrm{~h}$ simulated cycling time trial. J. Sports Sci. 1997, 15, 223-230. [CrossRef]

74. Finger, D.; Farinha, J.B.; Brusco, C.M.; Boeno, F.P.; Cadore, E.L.; Pinto, R.S.; Lanferdini, F.J.; Helal, L. Ingestion of carbohydrate or carbohydrate plus protein does not enhance performance during endurance exercise: A randomized crossover placebo-controlled clinical trial. Appl. Physiol. Nutr. Metab. 2018, 43, 937-944. [CrossRef]

75. Fulco, C.S.; Zupan, M.; Muza, S.R.; Rock, P.B.; Kambis, K.; Payn, T.; Hannon, M.; Glickman, E.; Cymerman, A. Carbohydrate supplementation and endurance performance of moderate altitude residents at $4300 \mathrm{~m}$. Int. J. Sports Med. 2007, 28, 437-443. [CrossRef]

76. Funnell, M.P.; Dykes, N.R.; Owen, E.J.; Mears, S.A.; Rollo, I.; James, L.J. Ecologically valid carbohydrate intake during soccerspecific exercise does not affect running performance in a fed state. Nutrients 2017, 9, 39. [CrossRef]

77. Ganio, M.S.; Klau, J.F.; Lee, E.C.; Yeargin, S.W.; McDermott, B.P.; Buyckx, M.; Maresh, C.M.; Armstrong, L.E. Effect of various carbohydrate-electrolyte fluids on cycling performance and maximal voluntary contraction. Int. J. Sport Nutr. Exerc. Metab. 2010, 20, 104-114. [CrossRef]

78. Glace, B.W.; Kremenic, I.J.; McHugh, M.P. Effect of carbohydrate beverage ingestion on central versus peripheral fatigue: A placebo-controlled, randomized trial in cyclists. Appl. Physiol. Nutr. Metab. 2019, 44, 139-147. [CrossRef]

79. Goedecke, J.H.; White, N.J.; Chicktay, W.; Mahomed, H.; Durandt, J.; Lambert, M.I. The effect of carbohydrate ingestion on performance during a simulated soccer match. Nutrients 2013, 5, 5193-5204. [CrossRef] [PubMed]

80. Gui, Z.; Sun, F.; Si, G.; Chen, Y. Effect of protein and carbohydrate solutions on running performance and cognitive function in female recreational runners. PLOS ONE 2017, 12, e0185982. [CrossRef] [PubMed]

81. Heesch, M.W.S.; Mieras, M.E.; Slivka, D.R. The performance effect of early versus late carbohydrate feedings during prolonged exercise. Appl. Physiol. Nutr. Metab. 2014, 39, 58-63. [CrossRef]

82. Hulston, C.J.; Jeukendrup, A.E. Substrate metabolism and exercise performance with caffeine and carbohydrate intake. Med. Sci. Sports Exerc. 2008, 40, 2096-2104. [CrossRef] [PubMed]

83. Hulston, C.J.; Jeukendrup, A.E. No placebo effect from carbohydrate intake during prolonged exercise. Int. J. Sport Nutr. Exerc. Metab. 2009, 19, 275-284. [CrossRef]

84. Ivy, J.L.; Miller, W.; Dover, V.; Goodyear, L.G.; Sherman, W.M.; Farrell, S.; Williams, H. Endurance improved by ingestion of a glucose polymer supplement. Med. Sci. Sports Exerc. 1983, 15, 466-471. [CrossRef] 
85. Jarvis, A.T.; Felix, S.D.; Sims, S.; Jones, M.T.; Anne, M.; Headley, S.A. Carbohydrate supplementation fails to improve the sprint performance of female cyclists. JEP Online 1999, 2, 16-23.

86. Jeukendrup, A.; Brouns, F.; Wagenmakers, A.J.M.; Saris, W.H.M. Carbohydrate-electrolyte feedings improve $1 \mathrm{~h}$ time trial cycling performance. Int. J. Sports Med. 1997, 18, 125-129. [CrossRef]

87. Kang, J.; Robertson, R.J.; Denys, B.G.; DaSilva, S.G.; Visich, P.; Suminski, R.R.; Utter, A.C.; Goss, F.L.; Metz, K.F. Effect of carbohydrate ingestion subsequent to carbohydrate supercompensation on endurance performance. Int. J. Sport Nutr. 1995, 5, 329-343. [CrossRef] [PubMed]

88. Kang, J.; Robertson, R.J.; Goss, F.L.; DaSilva, S.G.; Visich, P.; Suminski, R.R.; Utter, A.C.; Denys, B.C. Effect of carbohydrate substrate availability on ratings of perceived exertion during prolonged exercise of moderate intensity. Percept. Mot. Ski. 1996, 82, 495-506. [CrossRef] [PubMed]

89. Khanna, G.L.; Manna, I. Supplementary effect of carbohydrate-electrolyte drink on sports performance, lactate removal \& cardiovascular response of athletes. Indian J. Med. Res. 2005, 121, 665-669.

90. King, A.J.; O’Hara, J.P.; Morrison, D.J.; Preston, T.; King, R.F.G.J. Carbohydrate dose influences liver and muscle glycogen oxidation and performance during prolonged exercise. Physiol. Rep. 2018, 6, e13555. [CrossRef]

91. King, A.J.; O’Hara, J.P.; Arjomandkhah, N.C.; Rowe, J.; Morrison, D.J.; Preston, T.; King, R.F.G.J. Liver and muscle glycogen oxidation and performance with dose variation of glucose-fructose ingestion during prolonged (3 h) exercise. Eur. J. Appl. Physiol. 2019, 119, 1157-1169. [CrossRef]

92. Kingwell, B.; McKenna, M.J.; Sandstrom, E.R.; Hargreaves, M. Effect of glucose polymer ingestion on energy and fluid balance during exercise. J. Sports Sci. 1989, 7, 3-8. [CrossRef] [PubMed]

93. Learsi, S.K.; Ghiarone, T.; Silva-Cavalcante, M.D.; Andrade-Souza, V.A.; Ataide-Silva, T.; Bertuzzi, R.; de Araujo, G.G.; McConell, G.; Lima-Silva, A.E. Cycling time trial performance is improved by carbohydrate ingestion during exercise regardless of a fed or fasted state. Scand. J. Med. Sci. Sports 2019, 29, 651-662. [CrossRef]

94. Lugo, M.; Sherman, W.M.; Wimer, G.S.; Garleb, K. Metabolic responses when different forms of carbohydrate energy are consumed during cycling. Int. J. Sport Nutr. 1993, 3, 398-407. [CrossRef]

95. Martínez-Lagunas, V.; Ding, Z.; Bernard, J.R.; Wang, B.; Ivy, J.L. Added Protein Maintains Efficacy of a Low-Carbohydrate Sports Drink. J. Strength Cond. Res. 2010, 24, 48-59. [CrossRef]

96. Maughan, R.J.; Fenn, C.E.; Leiper, J.B. Effects of fluid, electrolyte and substrate ingestion on endurance capacity. Eur. J. Appl. Physiol. Occup. Physiol.1989, 58, 481-486. [CrossRef]

97. McConell, G.; Kloot, K.; Hargreaves, M. Effect of timing of carbohydrate ingestion on endurance exercise performance. Med. Sci. Sports Exerc. 1996, 28, 1300-1304. [CrossRef]

98. McConell, G.; Snow, R.J.; Proietto, J.; Hargreaves, M. Muscle metabolism during prolonged exercise in humans: Influence of carbohydrate availability. J. Appl. Physiol. 1999, 87, 1083-1086. [CrossRef]

99. McConell, G.K.; Canny, B.J.; Daddo, M.C.; Nance, M.J.; Snow, R.J. Effect of carbohydrate ingestion on glucose kinetics and muscle metabolism during intense endurance exercise. J. Appl. Physiol. 2000, 89, 1690-1698. [CrossRef] [PubMed]

100. McGawley, K.; Shannon, O.; Betts, J. Ingesting a high-dose carbohydrate solution during the cycle section of a simulated olympicdistance triathlon improves subsequent run performance. Appl. Physiol. Nutr. Metab. 2012, 37, 664-671. [CrossRef] [PubMed]

101. Millard-Stafford, M.; Sparling, P.B.; Rosskopf, L.B.; Hinson, B.T.; Dicarlo, L.J. Carbohydrate-electrolyte replacement during a simulated triathlon in the heat. Med. Sci. Sports Exerc. 1990, 22, 621-628. [CrossRef] [PubMed]

102. Millard-Stafford, M.L.; Sparling, P.B.; Rosskopf, L.B.; Snow, T.K. Should carbohydrate concentration of a sports drink be less than 8\% during exercise in the heat? Int. J. Sport Nutr. Exerc. Metab. 2005, 15, 117-130. [CrossRef]

103. Millard-Stafford, M.L.; Cureton, K.J.; Wingo, J.E.; Trilk, J.; Warren, G.L.; Buyckx, M. Hydration during exercise in warm, humid conditions: Effect of a caffeinated sports drink. Int. J. Sport Nutr. Exerc. Metab. 2007, 17, 163-177. [CrossRef]

104. Morris, J.G.; Nevill, M.E.; Thompson, D.; Collie, J.; Williams, C. The influence of a $6.5 \%$ carbohydrate-electrolyte solution on performance of prolonged intermittent high-intensity running at $30^{\circ} \mathrm{C}$. J. Sports Sci. 2003, 21, 371-381. [CrossRef]

105. Murray, R.; Eddy, D.E.; Murray, T.W.; Seifert, J.G.; Paul, G.L.; Halaby, G.A. The effect of fluid and carbohydrate feedings during intermittent cycling exercise. Med. Sci. Sports Exerc. 1987, 19, 597-604. [CrossRef]

106. Murray, R.; Seifert, J.G.; Eddy, D.E.; Paul, G.L.; Halaby, G.A. Carbohydrate feeding and exercise: Effect of beverage carbohydrate content. Eur. J. Appl. Physiol. Occup. Physiol. 1989, 59, 152-158. [CrossRef]

107. Murray, R.; Paul, G.L.; Seifert, J.G.; Eddy, D.E. Responses to varying rates of carbohydrate ingestion during exercise. Med. Sci. Sports Exerc. 1991, 23, 713-718. [CrossRef]

108. Naclerio, F.; Larumbe-Zabala, E.; Cooper, R.; Judith, A.; Alkhatib, A.; Baddeley-White, D.; Garcia, S.; Zanotto, T. Effect of a multi-ingredient supplement on intermittent sprint performance, fatigue perception, muscle damage and immunosuppression in recreational athletes. J. Int. Soc. Sports Nutr. 2014, 11, 1151-1158. [CrossRef]

109. Nassif, C.; Gomes, A.R.; Peixoto, G.H.C.; Chagas, M.H.; Soares, D.D.; Silami-Garcia, E.; Drinkwater, E.J.; Cannon, J.; Marino, F.E. The effect of double-Blind carbohydrate ingestion during $60 \mathrm{~km}$ of self-paced exercise in warm ambient conditions. PLoS ONE 2014, 9, e104710. [CrossRef] [PubMed]

110. Nassis, G.P.; Williams, C.; Chisnall, P. Effect of a carbohydrate-electrolyte drink on endurance capacity during prolonged intermittent high intensity running. Br. J. Sports Med. 1998, 32, 248-252. [CrossRef] [PubMed] 
111. Newell, M.L.; Hunter, A.M.; Lawrence, C.; Tipton, K.D.; Galloway, S.D.R. The ingestion of 39 or 64 g.h ${ }^{-1}$ of carbohydrate is equally effective at improving endurance exercise performance in cyclists. Int. J. Sport Nutr. Exerc. Metab. 2015, 25, 285-292. [CrossRef]

112. Nicholas, C.W.; Williams, C.; Lakomy, H.K.A.; Phillips, G.; Nowitz, A. Influence of ingesting a carbohydrate-electrolyte solution on endurance capacity during intermittent, high-intensity shuttle running. J. Sports Sci. 1995, 13, 283-290. [CrossRef] [PubMed]

113. Nikolopoulos, V.; Arkinstall, M.J.; Hawley, J.A. Reduced Neuromuscular Activity with Carbohydrate Ingestion during Constant Load Cycling. Int. J. Sport Nutr. Exerc. Metab. 2004, 14, 161-170. [CrossRef]

114. Nishibata, I.; Sadamoto, T.; Mutoh, Y.; Miyashita, M. Glucose ingestion before and during exercise does not enhance performance of daily repeated endurance exercise. Eur. J. Appl. Physiol. Occup. Physiol. 1993, 66, 65-69. [CrossRef]

115. Oosthuyse, T.; Carstens, M.; Millen, A.M.E. Ingesting isomaltulose versus fructose-maltodextrin during prolonged moderateheavy exercise increases fat oxidation but impairs gastrointestinal comfort and cycling performance. Int. J. Sport Nutr. Exerc. Metab. 2015, 25, 427-438. [CrossRef]

116. Oosthuyse, T.; Carstens, M.; Millen, A.M.E. Whey or Casein Hydrolysate with Carbohydrate for Metabolism and Performance in Cycling. Int. J. Sports Med. 2015, 36, 636-646. [CrossRef]

117. Osterberg, K.L.; Zachwieja, J.J.; Smith, J.W. Carbohydrate and carbohydrate + protein for cycling time-trial performance. J. Sports Sci. 2008, 26, 227-233. [CrossRef] [PubMed]

118. O'Hara, J.P.; Carroll, S.; Cooke, C.B.; King, R.F.G.J. The effect of pre-exercise galactose and glucose ingestion on high-intensity endurance cycling. J. Strength Cond. Res. 2014, 28, 2145-2153. [CrossRef] [PubMed]

119. O'Neal, E.K.; Poulos, S.P.; Wingo, J.E.; Richardson, M.T.; Bishop, P.A. Post-prandial carbohydrate ingestion during 1-h of moderateintensity, intermittent cycling does not improve mood, perceived exertion, or subsequent power output in recreationally-active exercisers. J. Int. Soc. Sports Nutr. 2013, 10, 4. [CrossRef]

120. Pettersson, S.; Edin, F.; Bakkman, L.; McGawley, K. Effects of supplementing with an $18 \%$ carbohydrate-hydrogel drink in $-5{ }^{\circ} \mathrm{C}$ with elite cross-country ski athletes: A crossover study. J. Int. Soc. Sports Nutr. 2019, 16, 46. [CrossRef] [PubMed]

121. Pottier, A.; Bouckaert, J.; Gilis, W.; Roels, T.; Derave, W. Mouth rinse but not ingestion of a carbohydrate solution improves 1-h cycle time trial performance. Scand. J. Med. Sci. Sports 2010, 20, 105-111. [CrossRef]

122. Riley, M.L.; Israel, R.G.; Holbert, D.; Tapscott, E.B.; Dohm, G.L. Effect of carbohydrate ingestion on exercise endurance and metabolism after a 1-day fast. Int. J. Sports Med. 1988, 9, 320-324. [CrossRef] [PubMed]

123. Roberts, J.D.; Tarpey, M.D.; Kass, L.S.; Tarpey, R.J.; Roberts, M.G. Assessing a commercially available sports drink on exogenous carbohydrate oxidation, fluid delivery and sustained exercise performance. J. Int. Soc. Sports Nutr. 2014, 11, 8. [CrossRef]

124. Robson-Ansley, P.; Barwood, M.; Eglin, C.; Ansley, L. The effect of carbohydrate ingestion on the interleukin-6 response to a 90-minute run time trial. Int. J. Sports Physiol. Perform. 2009, 4, 186-194. [CrossRef]

125. Robson-Ansley, P.; Walshe, I.; Ward, D. The effect of carbohydrate ingestion on plasma interleukin-6, hepcidin and iron concentrations following prolonged exercise. Cytokine 2011, 53, 196-200. [CrossRef]

126. Rollo, I.; Williams, C. Influence of ingesting a carbohydrate-electrolyte solution before and during a 1-hr running performance test. Int. J. Sport Nutr. Exerc. Metab. 2009, 19, 645-658. [CrossRef]

127. Rollo, I.; Williams, C. Influence of ingesting a carbohydrate-electrolyte solution before and during a 1-hour run in fed endurancetrained runners. J. Sports Sci. 2010, 28, 593-601. [CrossRef]

128. Rollo, I.; Williams, C.; Nevill, M. Influence of ingesting versus mouth rinsing a carbohydrate solution during a 1-h Run. Med. Sci. Sports Exerc. 2011, 43, 468-475. [CrossRef]

129. Smith, J.W.; Zachwieja, J.J.; Péronnet, F.; Passe, D.H.; Massicotte, D.; Lavoie, C.; Pascoe, D.D. Fuel selection and cycling endurance performance with ingestion of [13C]glucose: Evidence for a carbohydrate dose response. J. Appl. Physiol. 2010, 108, 1520-1529. [CrossRef] [PubMed]

130. Steiner, J.L.; Curmaci, A.; Patrie, J.T.; Gaesser, G.A.; Weltman, A. Effects of carbohydrate supplementation on the RPE-blood lactate relationship. Med. Sci. Sports Exerc. 2009, 41, 1326-1333. [CrossRef] [PubMed]

131. Sun, F.H.; Wong, S.H.S.; Chen, S.H.; Poon, T.C. Carbohydrate electrolyte solutions enhance endurance capacity in active females. Nutrients 2015, 7, 3739-3750. [CrossRef] [PubMed]

132. Tokmakidis, S.P.; Karamanolis, I.A. Effects of carbohydrate ingestion $15 \mathrm{~min}$ before exercise on endurance running capacity. Appl. Physiol. Nutr. Metab. 2008, 33, 441-449. [CrossRef]

133. Tsintzas, K.; Liu, R.; Williams, C.; Campbell, I.; Gaitanos, G. The effect of carbohydrate ingestion on performance during a 30-km race. Int. J. Sport Nutr. 1993, 3, 127-139. [CrossRef]

134. Tsintzas, O.K.; Willia, C.; Singh, R.; Wilson, W.; Burrin, J. Influence of carbohydrate-electrolyte drinks on marathon running performance. Eur. J. Appl. Physiol. Occup. Physiol. 1995, 70, 154-160. [CrossRef]

135. Tsintzas, O.K.; Williams, C.; Boobis, L.; Greenhaff, P. Carbohydrate ingestion and single muscle fiber glycogen metabolism during prolonged running in men. J. Appl. Physiol. 1996, 81, 801-809. [CrossRef]

136. Tsintzas, O.K.; Williams, C.; Wilson, W.; Burrin, J. Influence of carbohydrate supplementation early in exercise on endurance running capacity. Med. Sci. Sports Exerc. 1996, 28, 1373-1379. [CrossRef]

137. Utter, A.C.; Kang, J.; Robertson, R.J.; Nieman, D.C.; Chaloupka, E.C.; Suminski, R.R.; Piccinni, C.R. Effect of carbohydrate ingestion on ratings of perceived exertion during a marathon. Med. Sci. Sports Exerc. 2002, 34, 1779-1784. [CrossRef] [PubMed] 
138. Van Essen, M.; Gibala, M.J. Failure of protein to improve time trial performance when added to a sports drink. Med. Sci. Sports Exerc. 2006, 38, 1476-1483. [CrossRef] [PubMed]

139. Walton, P.T.; Rhodes, E.C. The effects of solid and liquid carbohydrate ingestion on high-intensity intermittent exercise performance. Biol. Sport 1997, 14, 45-54.

140. Watson, P.; Shirreffs, S.M.; Maughan, R.J. Effect of dilute $\mathrm{CHO}$ beverages on performance in cool and warm environments. Med. Sci. Sports Exerc. 2012, 44, 336-343. [CrossRef]

141. Wilber, R.L.; Moffatt, R.J. Influence of carbohydrate ingestion on blood glucose and performance in runners. Int. J. Sport Nutr. 1992, 2, 317-327. [CrossRef]

142. Williams, C.; Nute, M.G.; Broadbank, L.; Vinall, S. Influence of fluid intake on endurance running performance-A comparison between water, glucose and fructose solutions. Eur. J. Appl. Physiol. Occup. Physiol. 1990, 60, 112-119. [CrossRef]

143. Vandenbogaerde, T.J.; Hopkins, W.G. Effects of acute carbohydrate supplementation on endurance performance: A meta-analysis. Sports Med. 2011, 41, 773-792. [CrossRef]

144. Temesi, J.; Johnson, N.A.; Raymond, J.; Burdon, C.A.; O'Connor, H.T. Carbohydrate ingestion during endurance exercise improves performance in adults. J. Nutr. 2011, 141, 890-897. [CrossRef]

145. Stellingwerff, T.; Cox, G.R. Systematic review: Carbohydrate supplementation on exercise performance or capacity of varying durations. Appl. Physiol. Nutr. Metab. 2014, 39, 998-1011. [CrossRef]

146. Wilson, P.B. Does carbohydrate intake during endurance running improve performance? A critical review. J. Strength Cond. Res. 2016, 30, 3539-3559. [CrossRef]

147. Mitchell, J.B.; Costill, D.L.; Houmard, J.A.; Fink, W.J.; Robergs, R.A.; Davis, J.A. Gastric emptying influence of prolonged exercise and carbohydrate concentration. Med. Sci. Sports Exerc. 1989, 21, 269-374. [PubMed]

148. Shi, X.; Summers, R.W.; Schedl, H.P.; Flanagan, S.W.; Chang, R.; Gisolfi, C.V. Effects of carbohydrate type and concentration and solution osmolality on water absorption. Med. Sci. Sports Exerc. 1995, 27, 1607-1615. [CrossRef] [PubMed]

149. Rehrer, N.J.; Beckers, E.; Brouns, F.; Hoor ten, F.; Saris, W.H. Exercise and training effects on gastric emptying of carbohydrate beverages. Med. Sci. Sports Exerc. 1989, 21, 540-549. [CrossRef] [PubMed]

150. Brouns, F.; Senden, J.; Beckers, E.J.; Saris, W.H.M. Osmolarity does not affect the gastric emptying rate of oral rehydration solutions. J. Parenter. Enter. Nutr. 1995, 19, 403-406. [CrossRef]

151. Murray, R.; Bartoli, W.; Stofan, J.; Horn, M.; Eddy, D. A comparison of the gastric emptying characteristics of selected sports drinks. Int. J. Sport Nutr. 1999, 9, 263-274. [CrossRef]

152. Shi, X.; Horn, M.K.; Osterberg, K.L.; Stofan, J.R.; Zachwieja, J.J.; Horswill, C.A.; Passe, D.H.; Murray, R. Gastrointestinal discomfort during intermittent high-intensity exercise: Effect of carbohydrate-electrolyte beverage. Int. J. Sport Nutr. Exerc. Metab. 2004, 14, 673-683. [CrossRef]

153. Peters, H.P.; van Schelven, F.W.; Verstappen, P.A.; de Boer, R.W.; Bol, E.; Erich, W.B.; van der Togt, C.R.; de Vries, W.R. Gastrointestinal problems as a function of carbohydrate supplements and mode of exercise. Med. Sci. Sports Exerc. 1993, 25, 1211-1224. [CrossRef]

154. Peters, H.P.F.; Akkermans, L.M.A.; Bol, E.; Mosterd, W.L. Gastrointestinal Symptoms During Exercise: The Effect of Fluid Supplementation. Sports Med. 1995, 20, 65-76. [CrossRef]

155. de Oliveira, E.P.; Burini, R.C. Carbohydrate-dependent, exercise-induced gastrointestinal distress. Nutrients 2014, 6, 4191-4199. [CrossRef]

156. De Oliveira, E.P.; Burini, R.C.; Jeukendrup, A. Gastrointestinal complaints during exercise: Prevalence, etiology, and nutritional recommendations. Sports Med. 2014, 44, 79-85. [CrossRef]

157. Williams, M.; Rawson, E.; Branch, D. Nutrition for Health, Fitness and Sport, 11th ed.; McGraw-Hill Education: New York, NY, USA, 2017; ISBN 9780078021350.

158. Stellingwerff, T. Contemporary nutrition approaches to optimize elite marathon performance. Int. J. Sports Physiol. Perform. 2013, 8, 573-578. [CrossRef] [PubMed]

159. Carlsson, M.; Wahrenberg, V.; Carlsson, M.S.; Andersson, R.; Carlsson, T. Gross and delta efficiencies during uphill running and cycling among elite triathletes. Eur. J. Appl. Physiol. 2020, 120, 961-968. [CrossRef] [PubMed]

160. Tsintzas, K.; Williams, C. Human muscle glycogen metabolism during exercise. Effect of carbohydrate supplementation. Sports Med. 1998, 25, 7-23. [CrossRef] [PubMed]

161. Pfeiffer, B.; Stellingwerff, T.; Zaltas, E.; Hodgson, A.B.; Jeukendrup, A.E. Carbohydrate oxidation from a drink during running compared with cycling exercise. Med. Sci. Sports Exerc. 2011, 43, 327-334. [CrossRef] [PubMed]

162. Coggan, A.R.; Coyle, E.F. Carbohydrate ingestion during prolonged exercise: Effects on metabolism and performance. Exerc. Sport Sci. Rev. 1991, 19, 1-40. [CrossRef]

163. Coyle, E.F.; Montain, S.J. Carbohydrate and fluid ingestion during exercise: Are there trade-offs? Med. Sci. Sports Exerc 1992, 24, 671-678. [CrossRef]

164. Coyle, E.F. Carbohydrate feeding during exercise. Int. J. Sports Med. 1992, 13, 126-128. [CrossRef]

165. Coyle, E.F. Carbohydrate supplementation during exercise. J. Nutr. 1992, 122, 788-795. [CrossRef]

166. Gollnick, P.D.; Piehl, K.; Saltin, B. Selective glycogen depletion pattern in human muscle fibres after exercise of varying intensity and at varying pedalling rates. J. Physiol. 1974, 241, 45-57. [CrossRef] 
167. Currell, K.; Jeukendrup, A. Validity, Reliability and Sensitivity of Measures of Sporting Performance. Sports Med. 2008, 38, 297-316. [CrossRef]

168. Williams, C.; Rollo, I. Carbohydrate Nutrition and Team Sport Performance. Sports Med. 2015, 45, 13-22. [CrossRef] [PubMed]

169. Baker, L.B.; Rollo, I.; Stein, K.W.; Jeukendrup, A.E. Acute effects of carbohydrate supplementation on intermittent sports performance. Nutrients 2015, 7, 5733-5763. [CrossRef]

170. Berg, J.M.; Stryer, L.; Tymoczko, J.; Gatto, G. Biochemistry, 9th ed.; W.H. Freeman \& Co Ltd.: New York, NY, USA, 2019; ISBN 9781319114657.

171. Hargreaves, M.; Spriet, L.L. Skeletal muscle energy metabolism during exercise. Nat. Metab. 2020, 2, 817-828. [CrossRef] [PubMed]

172. Parolin, M.L.; Chesley, A.; Matsos, M.P.; Spriet, L.L.; Jones, N.L.; Heigenhauser, G.J.F. Regulation of skeletal muscle glycogen phosphorylase and PDH during maximal intermittent exercise. Am. J. Physiol.-Endocrinol. Metab. 1999, 277. [CrossRef] [PubMed]

173. Romijn, J.A.; Coyle, E.F.; Sidossis, L.S.; Gastaldelli, A.; Horowitz, J.F.; Endert, E.; Wolfe, R.R. Regulation of endogenous fat and carbohydrate metabolism in relation to exercise intensity and duration. Am. J. Physiol.-Endocrinol. Metab. 1993, 265, 380-391. [CrossRef] [PubMed]

174. Ishihara, K.; Uchiyama, N.; Kizaki, S.; Mori, E.; Nonaka, T.; Oneda, H. Application of Continuous Glucose Monitoring for Assessment of Individual Carbohydrate Requirement during Ultramarathon Race. Nutrients 2020, 12, 1121. [CrossRef]

175. Alfageme, R.J.; López, L.A.; Ayuso, J.M.; Martínez-Sanz, J.M. Analysis of nutritional intake in trail runners during competition. Nutr. Hosp. 2020, 38, 2. [CrossRef]

176. Costa, R.J.S.; Knechtle, B.; Tarnopolsky, M.; Hoffman, M.D. Nutrition for ultramarathon running: Trail, track, and road. Int. J. Sport Nutr. Exerc. Metab. 2019, 29, 130-140. [CrossRef]

177. Tiller, N.B.; Roberts, J.D.; Beasley, L.; Chapman, S.; Pinto, J.M.; Smith, L.; Wiffin, M.; Russell, M.; Sparks, S.A.; Duckworth, L.; et al. International Society of Sports Nutrition Position Stand: Nutritional considerations for single-stage ultra-marathon training and racing. J. Int. Soc. Sports Nutr. 2019, 16, 50. [CrossRef]

178. Martinez, S.; Aguilo, A.; Rodas, L.; Lozano, L.; Moreno, C.; Tauler, P. Energy, macronutrient and water intake during a mountain ultramarathon event: The influence of distance. J. Sports Sci. 2018, 36, 333-339. [CrossRef]

179. Friedlander, A.L.; Casazza, G.A.; Horning, M.A.; Huie, M.J.; Brooks, G.A. Training-induced alterations of glucose flux in men. J. Appl. Physiol. 1997, 82, 1360-1369. [CrossRef] [PubMed]

180. Coggan, A.R.; Kohrt, W.M.; Spina, R.J.; Bier, D.M.; Holloszy, J.O. Endurance training decreases plasma glucose turnover and oxidation during moderate-intensity exercise in men. J. Appl. Physiol. 1990, 68, 990-996. [CrossRef] [PubMed]

181. Bergman, B.C.; Horning, M.A.; Casazza, G.A.; Wolfel, E.E.; Butterfield, G.E.; Brooks, G.A. Endurance training increases gluconeogenesis during rest and exercise in men. Am. J. Physiol.-Endocrinol. Metab. 2000, 278, 244-251. [CrossRef] [PubMed]

182. Areta, J.L.; Hopkins, W.G. Skeletal Muscle Glycogen Content at Rest and During Endurance Exercise in Humans: A Meta-Analysis. Sports Med. 2018, 48, 2091-2102. [CrossRef]

183. Bergman, B.C.; Butterfield, G.E.; Wolfel, E.E.; Lopaschuk, G.D.; Casazza, G.A.; Horning, M.A.; Brooks, G.A. Muscle net glucose uptake and glucose kinetics after endurance training in men. Am. J. Physiol.-Endocrinol. Metab. 1999, 277, 81-92. [CrossRef]

184. Saunders, M.J.; Luden, N.D.; Herrick, J.E. Consumption of an oral carbohydrate-protein gel improves cycling endurance and prevents postexercise muscle damage. J. Strength Cond. Res. 2007, 21, 678-684. [CrossRef]

185. Devries, M.C. Sex-based differences in endurance exercise muscle metabolism: Impact on exercise and nutritional strategies to optimize health and performance in women. Exp. Physiol. 2016, 101, 243-249. [CrossRef]

186. Carter, S.L.; Rennie, C.; Tarnopolsky, M.A. Substrate utilization during endurance exercise in men and women after endurance training. Am. J. Physiol.-Endocrinol. Metab. 2001, 280, 898-907. [CrossRef]

187. Riddell, M.C.; Partington, S.L.; Stupka, N.; Armstrong, D.; Rennie, C.; Tarnopolsky, M.A. Substrate Utilization during Exercise Performed with and Without Glucose Ingestion in Female and Male Endurance-Trained Athletes. Int. J. Sport Nutr. Exerc. Metab. 2003, 13, 407-421. [CrossRef]

188. Devries, M.C.; Hamadeh, M.J.; Phillips, S.M.; Tarnopolsky, M.A. Menstrual cycle phase and sex influence muscle glycogen utilization and glucose turnover during moderate-intensity endurance exercise. Am. J. Physiol.-Regul. Integr. Comp. Physiol. 2006, 291, 1120-1128. [CrossRef]

189. Hargreaves, M. Exercise, muscle, and CHO metabolism. Scand. J. Med. Sci. Sports 2015, 25, 29-33. [CrossRef] [PubMed]

190. Forbes, S.C.; Candow, D.G.; Smith-ryan, A.E.; Hirsch, K.R.; Roberts, M.D.; Vandusseldorp, T.A.; Stratton, M.T.; Kaviani, M.; Little, J.P. Supplements and nutritional interventions to augment high-intensity interval training physiological and performance adaptations-A narrative review. Nutrients 2020, 12, 390. [CrossRef]

191. Kiens, B. Skeletal muscle lipid metabolism in exercise and insulin resistance. Physiol. Rev. 2006, 86, 205-243. [CrossRef] [PubMed]

192. Ferraris, R.P.; Diamond, J. Regulation of intestinal sugar transport. Physiol. Rev. 1997, 77, 257-302. [CrossRef] [PubMed]

193. Kellett, G.L. The facilitated component of intestinal glucose absorption. J. Physiol. 2001, 531, 585-595. [CrossRef] [PubMed]

194. Meyer, H.; Vitavska, O.; Wieczorek, H. Identification of an animal sucrose transporter. J. Cell Sci. 2011, 124, 1984-1991. [CrossRef]

195. Koivisto, V.A. Fructose as a Dietary Sweetener in Diabetes Mellitus. Diabetes Care 1978, 1, 241-246. [CrossRef]

196. Koivisto, V.A.; Karonen, S.L.; Nikkilä, E.A. Carbohydrate ingestion before exercise: Comparison between glucose and fructose. J. Appl. Physiol. Respir. Env. Exerc. Physiol. 1981, 40, 783-787. [CrossRef] 
197. Massicotte, D.; Péronnet, F.; Allah, C.; Hillaire-Marcel, C.; Ledoux, M.; Brisson, G. Metabolic response to [13C] glucose and [13C] fructose ingestion during exercise. J. Appl. Physiol. 1986, 61, 1180-1184. [CrossRef]

198. Massicotte, D.; Peronnet, F.; Brisson, G.; Bakkouch, K.; Hillaire-Marcel, C. Oxidation of a glucose polymer during exercise: Comparison with glucose and fructose. J. Appl. Physiol. 1989, 66, 179-183. [CrossRef]

199. Jandrain, B.J.; Pallikarakis, N.; Normand, S.; Pirnay, F.; Lacroix, M.; Mosora, F.; Pachiaudi, C.; Gautier, J.F.; Scheen, A.J.; Riou, J.P.; et al. Fructose utilization during exercise in men: Rapid conversion of ingested fructose to circulating glucose. J. Appl. Physiol. 1993, 74, 2146-2154. [CrossRef]

200. Adopo, E.; Peronnet, F.; Massicotte, D.; Brisson, G.R.; Hillaire-Marcel, C. Respective oxidation of exogenous glucose and fructose given in the same drink during exercise. J. Appl. Physiol. 1994, 76, 1014-1019. [CrossRef] [PubMed]

201. Jeukendrup, A.E. Carbohydrate and exercise performance: The role of multiple transportable carbohydrates. Curr. Opin. Clin. Nutr. Metab. Care 2010, 13, 452-457. [CrossRef] [PubMed]

202. Burelle, Y.; Peronnet, F.; Massicotte, D.; Brison, G.R.; Hillaire-Marcel, C. Oxidation of 13C-glucose and 13C-fructose ingested as a preexercise meal: Effect of carbohydrate ingestion during exercise. Int. J. Sport Nutr. 1997, 7, 117-127. [CrossRef] [PubMed]

203. Hawley, J.A.; Burke, L.M. Effect of meal frequency and timing on physical performance. Br. J. Nutr. 1997, 77, S91-S103. [CrossRef]

204. Hawley, J.A.; Dennis, S.C.; Noakes, T.D. Oxidation of Carbohydrate Ingested During Prolonged Endurance Exercise. Sports Med. 1992, 14, 27-42. [CrossRef]

205. Wagenmakers, A.J.M.; Brouns, F.; Saris, W.H.M.; Halliday, D. Oxidation rates of orally ingested carbohydrates during prolonged exercise in men. J. Appl. Physiol. 1993, 75, 2774-2780. [CrossRef] [PubMed]

206. Seiple, R.S.; Vivian, V.M.; Fox, L.; Bartels, R.L. Gastric emptying characteristics of two glucose polymer-electrolyte solutions. Med. Sci. Sports Exerc. 1983, 15, 366-369. [CrossRef]

207. Sole, C.C.; Noakes, T.D. Faster gastric emptying for glucose-polymer and fructose solutions than for glucose in humans. Eur. J. Appl. Physiol. Occup. Physiol. 1989, 58, 605-612. [CrossRef]

208. Moodley, D.; Noakes, T.D.; Bosch, A.N.; Hawley, J.A.; Schall, R.; Dennis, S.C. Oxidation of exogenous carbohydrate during prolonged exercise: The effects of the carbohydrate type and its concentration. Eur. J. Appl. Physiol. Occup. Physiol. 1992, 64, 328-334. [CrossRef]

209. Neufer, P.D.; Costill, D.L.; Fink, W.J.; Kirwan, J.P.; Fielding, R.A.; Flynn, M.G. Effects of exercise and carbohydrate composition on gastric emptying. Med. Sci. Sports Exerc. 1986, 18, 658-662. [CrossRef]

210. Foster, C.; Costill, D.L.; Fink, W.J. Gastric emptying characteristics of glucose and glucose polymer solutions. Res. Q. Exerc. Sport 1980, 51, 299-305. [CrossRef]

211. Jentjens, R.L.P.G.; Moseley, L.; Waring, R.H.; Harding, L.K.; Jeukendrup, A.E. Oxidation of combined ingestion of glucose and sucrose during exercise. J. Appl. Physiol. 2004, 96, 1277-1284. [CrossRef]

212. Jeukendrup, A.E. Carbohydrate feeding during exercise. Eur. J. Sport Sci. 2008, 8, 77-86. [CrossRef]

213. Jeukendrup, A.E.; Moseley, L.; Mainwaring, G.I.; Samuels, S.; Perry, S.; Mann, C.H. Exogenous carbohydrate oxidation during ultraendurance exercise. J. Appl. Physiol. 2006, 100, 1134-1141. [CrossRef] [PubMed]

214. Rowlands, D.S.; Swift, M.; Ros, M.; Green, J.G. Composite versus single transportable carbohydrate solution enhances race and laboratory cycling performance. Appl. Physiol. Nutr. Metab. 2012, 37, 425-436. [CrossRef]

215. Triplett, D.; Doyle, J.A.; Rupp, J.C.; Benardot, D. An isocaloric glucose-fructose beverage's effect on simulated 100-km cycling performance compared with a glucose-only beverage. Int. J. Sport Nutr. Exerc. Metab. 2010, 20, 122-131. [CrossRef]

216. Rowlands, D.S.; Houltham, S.; Musa-Veloso, K.; Brown, F.; Paulionis, L.; Bailey, D. Fructose-Glucose Composite Carbohydrates and Endurance Performance: Critical Review and Future Perspectives. Sports Med. 2015, 45, 1561-1576. [CrossRef] [PubMed]

217. Gisolfi, C.V. Use of electrolytes in fluid replacement solutions: What have we learned from intestinal absorption studies? In Fluid Replacement and Heat Stress; Marriott, B.M., Ed.; Institute of Medicine (US) Committee on Military Nutrition Research: Washington, DC, USA, 1994; pp. 11-22; ISBN 0309573459.

218. Gisolfi, C.V.; Duchman, S.M. Guidelines for optimal replacement beverages for different athletic events. Med. Sci. Sports Exerc. 1992, 24, 679-687. [CrossRef] [PubMed]

219. Sawka, M.N.; Burke, L.M.; Eichner, E.R.; Maughan, R.J.; Montain, S.J.; Stachenfeld, N.S. Exercise and fluid replacement. Med. Sci. Sports Exerc. 2007, 39, 377-390. [CrossRef]

220. Burke, L.M.; Jones, A.M.; Jeukendrup, A.E.; Mooses, M. Contemporary nutrition strategies to optimize performance in distance runners and race walkers. Int. J. Sport Nutr. Exerc. Metab. 2019, 29, 117-129. [CrossRef] [PubMed]

221. Burke, L.M.; Hawley, J.A.; Wong, S.H.S.; Jeukendrup, A.E. Carbohydrates for training and competition. J. Sports Sci. 2011, 29, S17-S27. [CrossRef] [PubMed]

222. Smith, J.E.W.; Zachwieja, J.J.; Horswill, C.A.; Pascoe, D.D.; Passe, D.; Ruby, B.C.; Stewart, L.K. Evidence of a Carbohydrate Dose and Prolonged Exercise Performance Relationship. Med. Sci. Sports Exerc. 2010, 42, 84. [CrossRef]

223. Jeukendrup, A. Carbohydrate during exercise: Research of last 10 years turned into new recommendations. Apunt. Educ. Física Deport. 2013, 113, 7-22. [CrossRef]

224. Smith, J.W.; Pascoe, D.D.; Passe, D.H.; Ruby, B.C.; Stewart, L.K.; Baker, L.B.; Zachwieja, J.J. Curvilinear dose-response relationship of carbohydrate $\left(0-120 \mathrm{~g} \cdot \mathrm{h}^{-1}\right)$ and performance. Med. Sci. Sports Exerc. 2013, 45, 336-341. [CrossRef]

225. Jeukendrup, A.E. Nutrition for endurance sports: Marathon, triathlon, and road cycling. J. Sports Sci. 2011, 29, S91-S99. [CrossRef] 
226. Jentjens, R.L.P.G.; Shaw, C.; Birtles, T.; Waring, R.H.; Harding, L.K.; Jeukendrup, A.E. Oxidation of combined ingestion of glucose and sucrose during exercise. Metabolism 2005, 54, 610-618. [CrossRef]

227. Currell, K.; Urch, J.; Cerri, E.; Jentjens, R.L.P.; Blannin, A.K.; Jeukendrup, A.E. Plasma deuterium oxide accumulation following ingestion of different carbohydrate beverages. Appl. Physiol. Nutr. Metab. 2008, 33, 1067-1072. [CrossRef]

228. Jeukendrup, A.E. Periodized Nutrition for Athletes. Sports Med. 2017, 47, 51-63. [CrossRef]

229. Lavoué, C.; Siracusa, J.; Chalchat, É.; Bourrilhon, C.; Charlot, K. Analysis of food and fluid intake in elite ultra-endurance runners during a 24-h world championship. J. Int. Soc. Sports Nutr. 2020, 17, 36. [CrossRef]

230. Yeo, S.E.; Jentjens, R.L.P.G.; Wallis, G.A.; Jeukendrup, A.E. Caffeine increases exogenous carbohydrate oxidation during exercise. J. Appl. Physiol. 2005, 99, 844-850. [CrossRef] [PubMed]

231. Pfeiffer, B.; Stellingwerff, T.; Zaltas, E.; Jeukendrup, A.E. CHO oxidation from a CHO Gel compared with a drink during exercise. Med. Sci. Sports Exerc. 2010, 42, 2038-2045. [CrossRef] [PubMed]

232. Jentjens, R.L.P.G.; Underwood, K.; Achten, J.; Currell, K.; Mann, C.H.; Jeukendrup, A.E. Exogenous carbohydrate oxidation rates are elevated after combined ingestion of glucose and fructose during exercise in the heat. J. Appl. Physiol. 2006, 100, 807-816. [CrossRef]

233. Pfeiffer, B.; Stellingwerff, T.; Zaltas, E.; Jeukendrup, A.E. Oxidation of solid versus liquid CHO sources during exercise. Med. Sci. Sports Exerc. 2010, 42, 2030-2037. [CrossRef] [PubMed]

234. Jeukendrup, A.E. Training the Gut for Athletes. Sports Med. 2017, 47, 101-110. [CrossRef]

235. Jeukendrup, A.E.; McLaughlin, J. Carbohydrate ingestion during exercise: Effects on performance, training adaptations and trainability of the gut. Nestle Nutr. Inst. Workshop Ser. 2011, 69, 1-17. [CrossRef]

236. Lesinski, M.; Hortobágyi, T.; Muehlbauer, T.; Gollhofer, A.; Granacher, U. Dose-Response Relationships of Balance Training in Healthy Young Adults: A Systematic Review and Meta-Analysis. Sports Med. 2015, 45, 557-576. [CrossRef]

237. Goulet, E.D.B.; Hoffman, M.D. Impact of Ad Libitum Versus Programmed Drinking on Endurance Performance: A Systematic Review with Meta-Analysis. Sports Med. 2019, 49, 221-232. [CrossRef] [PubMed]

238. Pöchmüller, M.; Schwingshackl, L.; Colombani, P.C.; Hoffmann, G. A systematic review and meta-analysis of carbohydrate benefits associated with randomized controlled competition-based performance trials. J. Int. Soc. Sports Nutr. $2016,13,27$. [CrossRef]

239. Brietzke, C.; Franco-Alvarenga, P.E.; Coelho-Júnior, H.J.; Silveira, R.; Asano, R.Y.; Pires, F.O. Effects of Carbohydrate Mouth Rinse on Cycling Time Trial Performance: A Systematic Review and Meta-Analysis. Sports Med. 2019, 49, 57-66. [CrossRef]

240. Thomas, J.R.; French, K.E. The use of meta-analysis in exercise and sport: A tutorial. Res. Q. Exerc. Sport 1986, 57, 196-204. [CrossRef]

241. Oosthuyse, T.; Bosch, A.N. The Effect of the Menstrual Cycle on Exercise Metabolism. Sports Med. 2010, 40, 207-227. [CrossRef] [PubMed]

242. Viribay, A.; Arribalzaga, S.; Mielgo-Ayuso, J.; Castañeda-Babarro, A.; Seco-Calvo, J.; Urdampilleta, A. Effects of 120 g/h of carbohydrates intake during a mountain marathon on exercise-induced muscle damage in elite runners. Nutrients 2020, $12,1367$. [CrossRef] [PubMed]

243. St. Clair Gibson, A.; Baden, D.A.; Lambert, M.I.; Lambert, E.V.; Harley, Y.X.R.; Hampson, D.; Russell, V.A.; Noakes, T.D. The conscious perception of the sensation of fatigue. Sports Med. 2003, 33, 167-176. [CrossRef]

244. Meeusen, R.; Watson, P.; Hasegawa, H.; Roelands, B.; Piacentini, M.F. Central fatigue: The serotonin hypothesis and beyond. Sports Med. 2006, 36, 881-909. [CrossRef] [PubMed]

245. Meeusen, R.; Piacentini, M.F. Exercise, fatigue, neurotransmission and the influence of the neuroendocrine axis. In Developments in Tryptophan and Serotonin Metabolism; Allegri, G., Costa, C.V.L., Ragazzi, E., Steinhart, H., Varesio, L., Eds.; Springer: Boston, MA, USA, 2003; Volume 527, pp. 521-525. ISBN 9781461501350.

246. Nybo, L.; Secher, N.H. Cerebral perturbations provoked by prolonged exercise. Prog. Neurobiol. 2004, 72, 223-261. [CrossRef] [PubMed]

247. Paris, H.L.; Fulton, T.J.; Chapman, R.F.; Fly, A.D.; Koceja, D.M.; Mickleborough, T.D. Effect of carbohydrate ingestion on central fatigue during prolonged running exercise in moderate hypoxia. J. Appl. Physiol. 2019, 126, 141-151. [CrossRef]

248. Nybo, L. CNS fatigue and prolonged exercise: Effect of glucose supplementation. Med. Sci. Sports Exerc. 2003, 35, 589-594. [CrossRef]

249. Nybo, L.; Møller, K.; Pedersen, B.K.; Nielsen, B.; Secher, N.H. Association between fatigue and failure to preserve cerebral energy turnover during prolonged exercise. Acta Physiol. Scand. 2003, 179, 67-74. [CrossRef]

250. Winnick, J.J.; Davis, J.M.; Welsh, R.S.; Carmichael, M.D.; Murphy, E.A.; Blackmon, J.A. Carbohydrates feedings during team sport exercise preserve physical and CNS function. Med. Sci. Sports Exerc. 2005, 37, 306-315. [CrossRef]

251. Kong, J.; Shepel, P.N.; Holden, C.P.; Mackiewicz, M.; Pack, A.I.; Geiger, J.D. Brain glycogen decreases with increased periods of wakefulness: Implications for homeostatic drive to sleep. J. Neurosci. 2002, 22, 5581-5587. [CrossRef] [PubMed]

252. Béquet, F.; Gomez-Merino, D.; Berthelot, M.; Guezennec, C.Y. Evidence that brain glucose availability influences exerciseenhanced extracellular 5-HT level in hippocampus: A microdialysis study in exercising rats. Acta Physiol. Scand. 2002, 176, 65-69. [CrossRef] [PubMed]

253. Jeffers, R.; Shave, R.; Ross, E.; Stevenson, E.J.; Goodall, S. The effect of a carbohydrate mouth-rinse on neuromuscular fatigue following cycling exercise. Appl. Physiol. Nutr. Metab. 2015, 40, 557-564. [CrossRef] [PubMed] 
254. Khong, T.K.; Selvanayagam, V.S.; Sidhu, S.K.; Yusof, A. Role of carbohydrate in central fatigue: A systematic review. Scand. J. Med. Sci. Sports 2017, 27, 376-384. [CrossRef]

255. Paris, H.L.; Sinai, E.C.; Shei, R.J.; Keller, A.M.; Mickleborough, T.D. The influence of carbohydrate ingestion on peripheral and central fatigue during exercise in hypoxia: A narrative review. Eur. J. Sport Sci. 2020, 21, 1423-1435. [CrossRef]

256. Malone, J.J.; Hulton, A.T.; MacLaren, D.P.M. Exogenous carbohydrate and regulation of muscle carbohydrate utilisation during exercise. Eur. J. Appl. Physiol. 2021, 121, 1255-1269. [CrossRef]

257. Manore, M.M.; Patton-Lopez, M.M.; Meng, Y.; Wong, S.S. Sport nutrition knowledge, behaviors and beliefs of high school soccer players. Nutrients 2017, 9, 350. [CrossRef]

258. Herriman, M.; Fletcher, L.; Tchaconas, A.; Adesman, A.; Milanaik, R. Dietary supplements and young teens: Misinformation and access provided by retailers. Pediatrics 2017, 139, e20161257. [CrossRef] 